quatrième série-tome 46 fascicule 6 novembre-décembre 2013

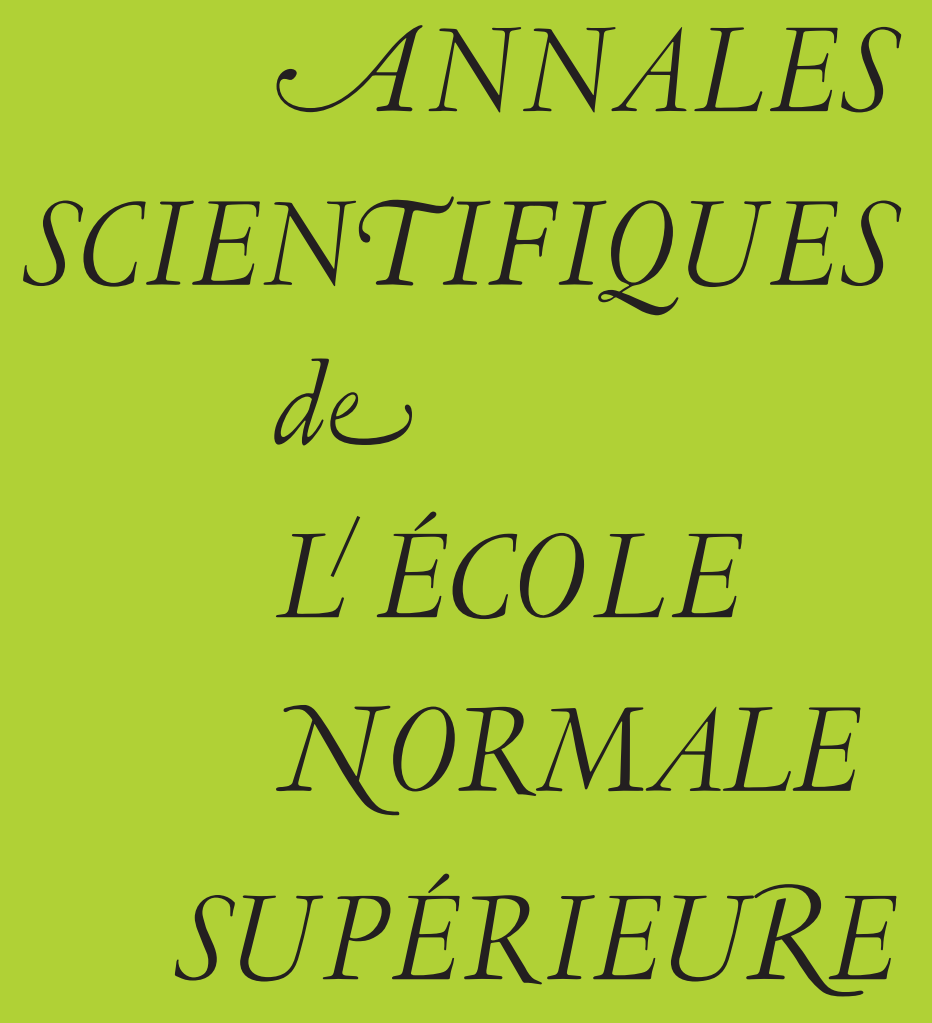

Nicolas BURQ\& Gilles LEBEAU

Injections de Sobolev probabilistes et applications 
Ann. Scient. Éc. Norm. Sup.

$4^{\text {e }}$ série, t. 46, 2013, p. 917 à 962

\title{
INJECTIONS DE SOBOLEV PROBABILISTES ET APPLICATIONS
}

\author{
PAR Nicolas BURQ et Gilles LEBEAU
}

RÉsumé. - On démontre dans cet article des versions probabilistes des injections de Sobolev sur une variété riemannienne compacte, $(M, g)$. Plus précisément on démontre que pour des mesures de probabilité naturelles sur l'espace $L^{2}(M)$, presque toute fonction appartient à tous les espaces $L^{p}(M)$, $p<+\infty$. On donne ensuite des applications à l'étude des harmoniques sphériques sur la sphère $\mathbb{S}^{d}$ : on démontre (encore pour des mesures de probabilité naturelles) que presque toute base hilbertienne de $L^{2}\left(\mathbb{S}^{d}\right)$ formée d'harmoniques sphériques a tous ses éléments uniformément bornés dans tous les espaces $L^{p}\left(\mathbb{S}^{d}\right), p<+\infty$. On démontre aussi des résultats similaires sur les tores $\mathbb{T}^{d}$. On donne aussi une application à l'étude du taux de décroissance de l'équation des ondes amortie dans un cadre où la condition de contrôle géométrique de Bardos, Lebeau et Rauch n'est pas vérifiée. En supposant le flot ergodique, on démontre qu'il existe sur des ensembles de mesure arbitrairement proche de 1 (dans l'espace des données initiales d'énergie finie), un taux de décroissance uniforme. Finalement, on conclut avec une application à l'étude de l'équation des ondes semi-linéaire $H^{1}$-surcritique, pour laquelle on démontre que pour presque toute donnée initiale, les solutions faibles sont fortes et uniques (localement en temps).

Abstract. - In this article, we give probabilistic versions of Sobolev embeddings on any Riemannian manifold $(M, g)$. More precisely, we prove that for natural probability measures on $L^{2}(M)$, almost every function belongs to all spaces $L^{p}(M), p<+\infty$. We then give applications to the study of the growth of the $L^{p}$ norms of spherical harmonics on spheres $\mathbb{S}^{d}$ : we prove (again for natural probability measures) that almost every Hilbert base of $L^{2}\left(\mathbb{S}^{d}\right)$ made of spherical harmonics has all its elements uniformly bounded in all $L^{p}\left(\mathbb{S}^{d}\right), p<+\infty$ spaces. We also prove similar results on tori $\mathbb{T}^{d}$. We give then an application to the study of the decay rate of damped wave equations in a framework where the geometric control property of Bardos-Lebeau-Rauch is not satisfied. Assuming that it is violated for a measure 0 set of trajectories, we prove that there exists almost surely a rate. Finally, we conclude with an application to the study of the $H^{1}$-supercritical wave equation, for which we prove that for almost all initial data, the weak solutions are strong and unique, locally in time. 


\section{Introduction}

L'objet de cet article est de démontrer que, si on choisit des fonctions au hasard sur une variété compacte, pour des mesures de probabilité naturelles, alors il est possible d'améliorer grandement les injections de Sobolev classiques. Plus précisément notre cadre est le suivant. Soit $(M, g)$ une variété riemannienne lisse compacte, sans bord connexe de dimension $d$ et $\boldsymbol{\Delta}$ le laplacien $\operatorname{sur}(M, g)$. Soit $0=\omega_{0}<\omega_{1} \leq \omega_{2} \leq \cdots$ le spectre de $\sqrt{-\boldsymbol{\Delta}}$ et $\left(e_{j}\right)_{j \geq 0}$ une base orthonormale $L^{2}$ de fonctions propres réelles, de sorte que $-\Delta e_{j}=\omega_{j}^{2} e_{j}$. Soient $0<a<b$ et $E_{h}$ le sous-espace de $L^{2}(M)$

$$
\left.\left.E_{h}=\left\{u=\sum_{k \in I_{h}} z_{k} e_{k}(x), z_{k} \in \mathbb{C}\right\}, \quad I_{h}=\left\{k, h \omega_{k} \in\right] a, b\right]\right\} .
$$

Soit $N_{h}=\operatorname{dim}\left(E_{h}\right)$. D'après la formule de Weyl, avec reste précisé (voir [18, Theorem $1.1])$, on a pour $h \in] 0,1]$

$$
N_{h}=(2 \pi h)^{-d} \operatorname{Vol}(M) \operatorname{Vol}\left(S^{d-1}\right) \int_{(a, b)} \rho^{d-1} d \rho+O\left(h^{-d+1}\right) .
$$

Rappelons qu'il existe une constante $C$ indépendante de $h \in] 0,1]$ telle qu'on a

$$
\|u\|_{L^{\infty}(M)} \leq C h^{-d / 2}\|u\|_{L^{2}(M)} \quad \forall u \in E_{h}
$$

et que plus généralement, si $A\left(x, h D_{x}\right)$ est un opérateur $h$-pseudodifférentiel classique sur $M$ de degré 0 et à support essentiel contenu dans $\left\{(x, \xi) \in T^{*} M,|\xi|_{x} \leq L\right\}$ pour un $L<\infty$, il existe une constante $C$ indépendante de $h \in] 0,1]$ telle que pour tout $1 \leq p \leq r \leq \infty$, on a

$$
\left\|A\left(x, h D_{x}\right) g\right\|_{L^{r}(M)} \leq C h^{-d\left(\frac{1}{p}-\frac{1}{r}\right)}\|g\|_{L^{p}(M)} \quad \forall g \in L^{p}(M) .
$$

Les inégalités de Sobolev (1.3) ou (1.4) sont optimales. L'objectif de cet article est d'étudier des versions probabilistes de ces inégalités. Décrivons rapidement le type de résultats que nous obtenons :

On note $S_{h}$ (resp. $\left.\widetilde{S}_{h}\right)$ la sphère unité de l'espace euclidien $E_{h}=\mathbb{C}^{N_{h}}\left(\right.$ resp. $\left.\widetilde{E}_{h}=\mathbb{R}^{N_{h}}\right)$, et $P_{h}$ (resp. $\widetilde{P}_{h}$ ) la probabilité uniforme sur $S_{h}$ (resp. $\widetilde{S}_{h}$ ). On verra dans la section 2 (voir en particulier le théorème 3) que les probabilités $P_{h}$ et $\widetilde{P}_{h}$ sont associées à une répartition uniforme de l'énergie dans l'espace de phase $T^{*} M$ pour la mesure de Liouville canonique $d \lambda$ sur $T^{*} M$. On notera $\mathbb{E}_{h}(f)=\int_{S_{h}} f(u) d P_{h}$ l'espérance d'une variable aléatoire $f$, et $\Pi_{h}$ le projecteur orthogonal de $L^{2}(M)$ sur $E_{h}$.

On a alors le résultat probabiliste essentiellement classique suivant, qui estime la mesure des $u \in E_{h}$ de grande norme $L^{\infty}$ (on pourra consulter Kahane [22] pour des résultats du même type).

ThÉorème 1. - Pour tout $c_{2}<\operatorname{Vol}(M)$, il existe $C>0$ tel que pour tout $\left.\left.h \in\right] 0,1\right]$ et tout $\Lambda \geq 1$ on ait, avec $c_{1}=d(1+d / 2)$

$$
\begin{aligned}
& P_{h}\left(u \in S_{h},\|u\|_{L^{\infty}}>\Lambda\right) \leq C h^{-c_{1}} e^{-c_{2} \Lambda^{2}}, \\
& \widetilde{P}_{h}\left(u \in \widetilde{S}_{h},\|u\|_{L^{\infty}}>\Lambda\right) \leq C h^{-c_{1}} e^{-c_{2} \Lambda^{2}} .
\end{aligned}
$$


Nous donnerons une preuve du théorème 1 dans la section 2. L'estimation (1.5) a une conséquence immédiate sur les versions probabilistes des injections de Sobolev. Rappelons que pour $p \in[1, \infty]$ et $s \geq 0$, l'espace de Sobolev $W^{s, p}$ est défini par

$$
W^{s, p}=\left\{f \in L^{p}(M),(1-\boldsymbol{\Delta})^{s / 2} f \in L^{p}(M)\right\} .
$$

Les espaces $W^{s, p}$ sont indépendants du choix de la métrique $g$ sur $\mathrm{M}$ et, pour $1 \leq p \leq r<\infty$, on a les injections de Sobolev

$$
W^{s, p} \subset L^{r}, \quad s=\frac{d}{p}-\frac{d}{r} .
$$

Rappelons aussi la construction de Littlewood-Paley. On fixe $0<a<c$, et $\varphi \in C^{\infty}(\mathbb{R})$ tel que $\varphi(t)=0$ pour $t \leq a, \varphi(t)=1$ pour $t \geq c$, et $\varphi^{\prime}(t)>0$ pour $\left.t \in\right] a, c[$. On pose $\psi_{-1}(t)=1-\varphi(t), \psi(t)=\varphi(t)-\varphi(t / 2), \psi_{n}(t)=\psi\left(2^{-n} t\right)$ pour $n \geq 0$. Alors $\psi$ est à support dans $[a, 2 c], \psi(t)>0$ pour $t \in] a, 2 c\left[\right.$ et $1=\sum_{n \geq-1} \psi_{n}(t)$ pour tout $t$. Posons $b=2 c>a>0$. Pour toute distribution $f \in \mathscr{D}^{\prime}(M)$, on a $f=\sum c_{k}(f) e_{k}$, où les $c_{k}(f)=\int_{M} f e_{k} d x$ sont les coefficients de Fourier de $f$, et la décomposition de LittlewoodPaley de f s'écrit, avec $h_{k}=2^{-k}$,

$$
f=\sum_{n=-1}^{\infty} f_{n}, \quad f_{n}=\psi_{n}(\sqrt{|\boldsymbol{\Delta}|}) f=\sum_{k} \psi_{n}\left(\omega_{k}\right) c_{k}(f) e_{k}, f_{n} \in E_{h_{n}}(n \geq 0) .
$$

Rappelons que, pour $q, r \in[1, \infty]$ et $s \in \mathbb{R}$, l'espace de Besov $B_{q, r}^{s}$ est l'espace des distributions $f \in \mathscr{D}^{\prime}(M)$ dont la décomposition de Littlewood-Paley vérifie

$$
\text { la suite } n \rightarrow 2^{n s}\left\|f_{n}\right\|_{L^{q}(M)} \text { appartient à } l^{r}(\mathbb{N}) \text {. }
$$

Les éléments de $E_{h_{n}}$ sont des fonctions à échelle $h_{n}=2^{-n}$ sur $M$, et les injections de Sobolev (1.7) peuvent être vues comme conséquence des inégalités (1.4).

Soit alors $X$ l'espace produit

$$
X=\Pi_{n=0}^{\infty} S_{h_{n}} .
$$

On munit $X$ de la probabilité produit $\mathbb{P}=\Pi_{n=0}^{\infty} P_{h_{n}}$. Soit $\left(a_{n}\right)_{n \geq 0}$ une suite de réels positifs telle que $\sum_{n} n^{1 / 2} a_{n}<\infty$. Soit $j$ l'application de $X$ dans l'espace de Besov $B_{2, \infty}^{0}$

$$
\begin{gathered}
X \rightarrow B_{2, \infty}^{0} \\
g=\left(g_{n}\right)_{n \geq 0} \mapsto j(g)=\sum_{n=0}^{\infty} a_{n} g_{n} .
\end{gathered}
$$

Comme corollaire immédiat du théorème 1 , on obtient

Corollaire 1.1. - On a $\mathbb{P}\left(j(g) \in C^{0}(M)\right)=1$.

Remarque 1.2. - On notera que le corollaire 1.1 est violent, puisqu'il implique en particulier une injection presque sûre de $B_{2, \infty}^{\sigma}$ dans $C^{0}(M)$ pour tout $\sigma>0$, soit un gain de $d / 2$ dérivées par rapport à l'injection de Sobolev. 
Démonstration. - Soit $A>0$ donné, $m_{n}=(A n \log (2))^{1 / 2}$ et $B_{n}$ la partie de $S_{2^{-n}}$

$$
B_{n}=\left\{g_{n},\left\|g_{n}\right\|_{L^{\infty}} \leq m_{n}\right\} \text {. }
$$

D'après (1.5) on a

$$
P_{h_{n}}\left(B_{n}\right) \geq 1-C 2^{-n\left(c_{2} A-c_{1}\right)} .
$$

Soit $B$ la partie de $X, B=S_{h_{0}} \times \Pi_{n=1}^{\infty} B_{n}$. Pour $g=\left(g_{n}\right) \in B$ et $f=j(g)$, on a

$$
\|f\|_{L^{\infty}} \leq \sum_{n=0}^{\infty} a_{n}\left\|g_{n}\right\|_{L^{\infty}} \leq C a_{0}+(A \log (2))^{1 / 2} \sum_{n=1}^{\infty} n^{1 / 2} a_{n} .
$$

On a alors pour tout $f \in j(B), f \in C^{0}(M)$ d'après (1.13), puisque les $g_{n}$ sont continus, et d'après (1.12)

$$
\mathbb{P}(B)=\prod_{n=1}^{\infty} P_{h_{n}}\left(B_{n}\right) \geq \prod_{n=1}^{\infty}\left(1-C 2^{-n\left(c_{2} A-c_{1}\right)}\right) \geq 1-\varepsilon,
$$

avec $\varepsilon>0$ petit si la constante $A$ est assez grande, d'où le résultat.

La morale du corollaire 1.1 est la suivante : si on se donne une famille de fonctions $g_{h} \in E_{h}$ à échelle $h$ et d'énergie 1 pour tout $h=2^{-n}$, et si on re-répartit leur énergie aléatoirement dans l'espace de phase, on obtient une nouvelle famille de fonctions dans $E_{h}$ qui est « presque » bornée dans le sens où $\sup _{h}\left\|g_{h}\right\|_{L^{\infty}}|\log (h)|^{-1 / 2}$ l'est .

Nos constructions de mesures sur l'espace $L^{2}(M)$ (voir l'appendice C pour la construction précise) utilisent une décomposition orthogonale $L^{2}(M)=\oplus_{k} E_{k}$, où les $E_{k}$ sont des sous-espaces de dimensions finies invariants par l'opérateur $\Delta$. On choisit en particulier sur chaque $E_{k}$ une probabilité $P_{k}$ invariante par les isométries de $E_{k}$, et on munit l'espace $L^{2}$ de la probabilité produit $P=\Pi_{k} P_{k}$. Dans notre cadre, si $\omega$ est la fréquence typique des éléments de $E_{k}$, on a toujours $C_{1} \omega^{d-1} \leq \operatorname{dim}\left(E_{k}\right) \leq C_{2} \omega^{d}$, et plus précisément, les fréquences $\omega$ des éléments de $E_{k}$ vérifient $\omega \in\left(a_{k}, b_{k}\right), a_{k}+C \leq b_{k} \leq c a_{k}$, avec $C>0, c>1$. Le fait de choisir des espaces $E_{k}$ de " grande dimension » permet d'obtenir des résultats plus forts avec probabilité 1 que le choix $E_{k}=\mathbb{C} e_{k}$, qui vérifie $\operatorname{dim}\left(E_{k}\right)=1$, et pour lequel nous renvoyons aux travaux de N. Tzvetkov [3], [35] et [36]. De plus, on verra dans la section 2 comment le choix que nous faisons des $E_{k}$ permet de relier naturellement nos probabilités à la mesure de Liouville sur $T^{*} M$.

L'article est organisé comme suit. Dans la section 2 nous démontrons le théorème 1 et des versions précisées, en autorisant des localisations spectrales plus fines que (1.1). Le théorème 3 de la section 2.2 précise le fait que nos mesures sont associées à la mesure de Liouville sur $T^{*} M$. Dans la section 2.3 nous décrivons pour $2<q \leq \infty$ les estimations $L^{q}$ presque sûres. On trouvera dans Shiffman-Zelditch [29] des preuves analogues pour les estimées sur les sections de fibrés holomorphes. Les bornes inférieures que nous obtenons sur les médianes des normes $L^{q}$ semblent nouvelles. Dans les sections suivantes, nous donnons des applications simples à l'étude de solutions d'équations aux dérivées partielles. Notre première application (dans la section 3 ) concerne la croissance des normes $L^{p}$ des harmoniques sphériques (les fonctions propres du laplacien sur les spheres $\mathbb{S}^{d} \subset \mathbb{R}^{d+1}$ ). Il est connu depuis les travaux de Hörmander [18] et de Sogge [32] que sur toute variété riemannienne compacte de dimension $d,(M, g)$, les fonctions propres du laplacien vérifient les estimations suivantes. 
ThÉORÈme. - Pour tout $2 \leq p \leq+\infty$, il existe $C>0$ tel que pour toutes fonctions propres du laplacien, $u,-\boldsymbol{\Delta}_{g} u=\lambda^{2} u$, on $a$

$$
\|u\|_{L^{p}(M)} \leq C \lambda^{\delta(p)}\|u\|_{L^{2}(M)}
$$

avec

$$
\delta(p)= \begin{cases}\frac{(d-1)}{2}-\frac{d}{p} & \text { si } p \geq \frac{2(d+1)}{d-1} \\ \frac{(d-1)}{2}\left(\frac{1}{2}-\frac{1}{p}\right) & \text { si } p \leq \frac{2(d+1)}{d-1}\end{cases}
$$

On sait par ailleurs que ces estimées sont optimales sur les sphères (munies de leur métrique standard). Dans le premier régime, les harmoniques sphériques zonales (qui se concentrent en deux points diamétralement opposés) réalisent l'optimum tandis que, dans le second, ce sont les harmoniques qui se concentrent sur un équateur qui saturent les estimées (1.14). Notre première application (voir Théorème 6 pour un énoncé plus précis) montre que ces harmoniques sphériques sont exceptionnelles.

THÉORÈME. - Considérons la mesure de probabilité naturelle (voir section 3) sur l'espace des bases hilbertiennes de $L^{2}\left(\mathbb{S}^{d}\right)$ formées d'harmoniques sphériques. Alors avec probabilité 1 , pour tout $p<+\infty$, toutes les normes $L^{p}$ sont bornées (uniformément). Autrement dit, on peut prendre $\delta(p)=0$ dans $(1.14)$ avec probabilité 1 .

Des questions similaires ont été étudiées par VanderKam [37] et Zelditch [38, 39]. On remarquera que ce phénomène d'existence de familles de fonctions propres exhibant des comportements différents en ce qui concerne la croissance des normes $L^{p}$ n'est pas si surprenant puisqu'il se manifeste aussi sur les tores $\mathbb{T}^{d}$. En effet, dans ce cadre la situation est renversée puisque les fonctions propres naturelles $\left(e^{i n \cdot x}, n \in \mathbb{Z}^{d}\right)$ ont toutes leurs normes $L^{p}$ bornées. Cependant, on démontre facilement (voir par exemple la section 3.1) qu'il existe sur $\mathbb{T}^{d}$ une suite de fonctions propres du laplacien $u_{n}$ vérifiant

$$
\left\|u_{n}\right\|_{L^{p}\left(\mathbb{T}^{d}\right)} \geq\left|\lambda_{n}\right|^{\frac{d-2}{2}-\frac{d}{p}}
$$

et donc, pour $d \geq 3, p \geq 2 d /(d-2)$, les normes $L^{p}$ ne sont pas uniformément bornées (voir [8] pour des majorations sur $\left.\mathbb{T}^{d}\right)$. On renvoie à $[8,9,10]$ pour de nombreux autres résultats sur les fonctions propres du laplacien sur les tores.

Notre deuxième application (section 4) concerne l'étude de l'équation des ondes amorties sur une variété compacte. On considère donc pour $a \in C^{\infty}(M ;[0,+\infty[)$ les solutions de

$$
\left(\partial_{t}^{2}-\boldsymbol{\Delta}\right) u+a(x) \partial_{t} u=0,\left.\quad\left(u, \partial_{t} u\right)\right|_{t=0}=\left(u_{0}, u_{1}\right) \in H^{1}(M) \times L^{2}(M) .
$$

Leur énergie

$$
\mathcal{E}(u)(t)=\frac{1}{2} \int_{M}\left(\left|\nabla_{x} u\right|^{2}+\left|\partial_{t} u\right|^{2}\right) d x
$$

vérifie

$$
\frac{d \mathscr{E}(t)}{d t}=-\int_{M} a(x)\left|\partial_{t} u\right|^{2} d x
$$

et est donc une fonction décroissante dont on peut démontrer qu'elle tend vers 0 quand $t$ tend vers l'infini dès que l'amortissement $a$ est non trivial. Si de plus il existe un taux de 
décroissance uniforme par rapport à l'énergie initiale, la propriété de semi-groupe montre que ce taux est alors toujours exponentiel :

$$
\mathcal{E}(u)(t) \leq C e^{-c t} \mathcal{E}(u)(0)
$$

ThÉorème (Bardos-Lebeau-Rauch [4]). - Il existe un taux de décroissance (exponentiel) uniforme si et seulement si toutes les géodésiques de la variété $M$ rencontrent la région a $>0$.

Ici, on s'intéresse à des situations où cette propriété géométrique n'est plus vérifiée, mais où elle est violée pour " un ensemble rare de géodésiques ». On démontre alors le résultat suivant (voir théorème 13 pour un énoncé plus précis).

ThÉorème. - On note G l'ensemble des points de l'espace des phases tels que le long de la géodésique issue de ce point, la moyenne asymptotique de l'amortissement est nulle. On supposera que la mesure dans l'espace des phases de G est nulle (on remarquera que si le flot est ergodique, cette propriété est vérifiée pour tous les amortissements non triviaux). Alors il existe un taux de décroissance $f(t), \lim _{t \rightarrow+\infty} f(t)=0$, tel que pour une large famille de mesures de probabilités $\mathcal{M}$ sur l'espace d'énergie $H^{1}(M) \times L^{2}(M)$, on a pour tout $\mathbb{P} \in \mathcal{M}$,

$$
\mathbb{P}\left(\left\{u \in H^{1}(M) \times L^{2}(M) ; \exists T, \mathcal{E}(U(t) u) \leq f(t) \forall t \geq T\right\}\right)=1 .
$$

Finalement, notre dernière application (section 5) concerne la théorie de Cauchy pour l'équation des ondes semilinéaire sur une variété compacte de dimension 3.

$$
\left(\partial_{t}^{2}-\boldsymbol{\Delta}\right) u+u^{p}=0,\left(\left.u\right|_{t=0},\left.\partial_{t} u\right|_{t=0}\right)=\left(u_{0}, u_{1}\right) \in\left(H^{1}(M) \cap L^{p+1}(M)\right) \times L^{2}(M),
$$

où $p$ est un entier impair. On connaît pour ce système l'existence de solutions faibles globales en temps. De plus, pour $p \leq 5$, ces solutions sont fortes et uniques. Nous démontrons le résultat suivant (voir les théorèmes 14 et 15 pour des énoncés plus précis)

ThÉORÈme. - Pour une large famille de mesures de probabilités naturelles sur l'espace des données initiales $\left(u_{0}, u_{1}\right) \in H^{1}(M) \times L^{2}(M)$, $\mathcal{M}$, il existe pour presque toute donnée initiale $\left(u_{0}, u_{1}\right)$ et tout $p<+\infty$ une solution locale forte (en un sens qui sera précisé) et sur l'intervalle d'existence de ces solutions fortes, il y a unicité des solutions faibles d'énergie décroissante (i.e. toute solution faible coüncide avec cette solution forte).

Certains de nos résultats restent vrais sur une variété à bord. Dans une dernière section, nous donnons les éléments permettant dans ce cadre d'adapter les démonstrations. Finalement, nous avons rassemblé dans un appendice quelques résultats de calcul des probabilités et de calcul pseudo-différentiel nécessaires à la compréhension de l'article. Nous souhaitons remercier M. Ledoux et P. Gérard pour des commentaires sur certains aspects du texte et les deux rapporteurs pour les remarques et suggestions qui ont permis d'améliorer substantiellement la présentation de l'article

Ce projet a bénéficié du soutien de l'Agence Nationale de la Recherche, projet ANR-07BLAN-0250

$4{ }^{\text {e }}$ SÉRIE - TOME $46-2013$ - No 6 


\section{Estimations probabilistes}

Dans cette section, nous calculons les lois de certaines variables aléatoires associées à la théorie de Littlewood-Paley sur la variété $M$. Les asymptotiques de Weyl jouent un rôle clé dans ces calculs. Nos résultats autorisent des localisations en fréquence plus fines et plus générales que les localisations dyadiques de l'introduction. Plus précisément, on considérera $0<a_{h}<b_{h} \leq c$ deux fonctions définies pour $h \in\left(0, h_{0}\right)$ telles que

$$
\lim _{h \rightarrow 0} b_{h}=b \geq \lim _{h \rightarrow 0} a_{h}=a \geq 0 .
$$

On supposera que si $a=b$, alors $a>0$ et

$$
b_{h}-a_{h} \geq D h
$$

pour une constante $D$ assez grande (à préciser ultérieurement). On notera $E_{h}$ (resp $\widetilde{E}_{h}$ ) le sous-espace de $L^{2}(M)$

$$
\begin{gathered}
E_{h}=\left\{u=\sum_{k \in I_{h}} z_{k} e_{k}(x), z_{k} \in \mathbb{C}\right\}, \quad \widetilde{E}_{h}=\left\{u=\sum_{k \in I_{h}} z_{k} e_{k}(x), z_{k} \in \mathbb{R}\right\} \\
\left.\left.I_{h}=\left\{k \in \mathbb{N} ; h \omega_{k} \in\right] a_{h}, b_{h}\right]\right\}
\end{gathered}
$$

Soit $N_{h}=\operatorname{dim}\left(E_{h}\right)$. Rappelons que, d'après la formule de Weyl, avec reste précisé (1.2) (voir Hörmander [18]), on a pour $h \in] 0,1]$, avec $c_{d}=\operatorname{Vol}\left(x \in \mathbb{R}^{d},|x| \leq 1\right)$ le volume de la boule unité en dimension $d$,

$$
\exists C>0 ; \forall \lambda>0\left|\sharp\left\{k \in \mathbb{N} ; \omega_{k} \leq \lambda\right\}-c_{d} \frac{\operatorname{Vol}(M)}{(2 \pi)^{d}} \lambda^{d}\right| \leq \lambda^{d-1},
$$

et donc

$$
\left|\sharp\left\{k ; \omega_{k} \in I_{h}\right\}-c_{d} \frac{\operatorname{Vol}(M)}{(2 \pi)^{d}}\left(\left(h^{-1} b_{h}\right)^{d}-\left(h^{-1} a_{h}\right)^{d}\right)\right| \leq C h^{-d+1},
$$

(2.6) $N_{h}=\sharp\left\{k ; \omega_{k} \in I_{h}\right\}$

$$
\sim \begin{cases}c_{d} \frac{\operatorname{Vol}(M)}{(2 \pi)^{d}}\left(b_{h}^{d}-a_{h}^{d}\right) h^{-d}, & \text { si } 0 \leq a<b, \\ c_{d} \frac{\operatorname{Vol}(M)}{(2 \pi)^{d}} d a^{d-1} h^{-d}\left[\left(b_{h}-a_{h}\right)+\theta\left(h+\left(b_{h}-a_{h}\right)^{2}+\left(b_{h}-a_{h}\right)\left|a-a_{h}\right|\right)\right], & \text { si } 0<a=b .\end{cases}
$$

On en déduit

$$
\begin{gathered}
\exists D_{0}>0 ; b_{h}-a_{h} \geq D_{0} h \Rightarrow \exists \beta>\alpha>0 ; \\
\alpha h^{-d}\left(b_{h}-a_{h}\right) \leq N_{h}=\sharp\left\{k ; \omega_{k} \in I_{h}\right\} \leq \beta h^{-d}\left(b_{h}-a_{h}\right) .
\end{gathered}
$$

Nous supposerons dans la suite que la constante $D$ dans (2.2) est choisie de telle façon que (2.7) soit vérifiée, et que l'on ait aussi pour tout $h$

$$
N_{h} \geq 2 \text {. }
$$

On munit les sphères unité de $E_{h}$ (resp. $\widetilde{E}_{h}$ ) de la mesure de probabilité uniforme, $P_{h}$ (resp. $\widetilde{P}_{h}$ ). 


\subsection{Estimations $L^{\infty}$ presque sûres}

Dans le cadre que nous venons de développer, le théorème 1 est un cas particulier du

ThÉORÈme 2. - Il existe $C>0, c_{2}>0$ tel que pour tout $\left.\left.h \in\right] 0,1\right]$ et tout $\Lambda \geq 1$ on ait, avec $c_{1}=d(1+d / 2)$

$$
\begin{aligned}
& P_{h}\left(u \in S_{h},\|u\|_{L^{\infty}}>\Lambda\right) \leq C h^{-c_{1}} e^{-c_{2} \Lambda^{2}} \\
& \widetilde{P}_{h}\left(u \in \widetilde{S}_{h},\|u\|_{L^{\infty}}>\Lambda\right) \leq C h^{-c_{1}} e^{-c_{2} \Lambda^{2}} .
\end{aligned}
$$

De plus, on peut choisir $\left.c_{2} \in\right] 0, \operatorname{Vol}(M)\left[\right.$ dans le cas $\lim _{h \rightarrow 0}\left(b_{h}-a_{h}\right) / h=+\infty$.

On se limitera dans la preuve au cas complexe, le cas réel étant similaire. Pour tout $x \in M$, et tout $\lambda \in \mathbb{R}^{+}$, on note

$$
\begin{gathered}
E_{x, \lambda}=\sum_{k ; \omega_{k} \leq \lambda}\left|e_{k}(x)\right|^{2}, \quad e_{x, h}=E_{x, h^{-1} b_{h}}-E_{x, h^{-1} a_{h}} \\
b_{x, h}=\left(e_{k}(x)\right)_{k \in I_{h}} \in \mathbb{C}^{N_{h}} .
\end{gathered}
$$

On a $e_{x, h}=\left|b_{x, h}\right|^{2}$. Soit $e v_{x}$ la variable aléatoire sur $\left(S_{h}, P_{h}\right)$ définie pour $a \in S_{h}$ par

$$
e v_{x}(u)=u(x)=\sum_{k \in I_{h}} z_{k} e_{k}(x)=\left(z \mid b_{x, h}\right)=\left(z \mid \frac{b_{x, h}}{\left|b_{x, h}\right|}\right)\left|b_{x, h}\right| \text {. }
$$

Le vecteur $\varepsilon_{x}=\frac{b_{x, h}}{\left|b_{x, h}\right|}$ est de norme 1 et on a pour tout $r \geq 0$, avec $P=P_{h}$

$$
P_{h}\left(\left|e v_{x}\right|>r\right)=P_{h}\left(\left|\left(z \mid \varepsilon_{x}\right)\right|>r /\left|b_{x, h}\right|\right)=\Phi\left(r /\left|b_{x, h}\right|\right),
$$

avec $\Phi(t)=P_{h}(|(z \mid \varepsilon)|>t)$ où $\varepsilon$ est un vecteur unitaire quelconque. D'après (A.6) (on identifie ici la sphère unité de $\mathbb{C}^{N_{h}}$ avec celle de $\mathbb{R}^{2 N_{h}}$ ), on a

$$
\Phi(t)=\mathbf{1}_{t \in[0,1[}\left(1-t^{2}\right)^{N_{h}-1} .
$$

Lemme 2.1. - Il existe $C_{0}>0$ tel que pour tout $x \in M$ et tout $\left.\left.h \in\right] 0,1\right]$ on a

$$
\left|e_{x, h}-\frac{N_{h}}{\operatorname{Vol}(M)}\right| \leq C_{0} h^{-d+1} .
$$

En particulier, si $a_{h}, b_{h}$ vérifient $b_{h}-a_{h} \geq D$ h avec D assez grand, d'après (2.6) et (2.7), on a avec $C$ indépendant de $x \in M$ et $h \in] 0,1]$

$$
N_{h} / C \leq e_{x, h} \leq C N_{h} .
$$

Démonstration. - D'après [18, Théorème 1.1], on a avec $C$ indépendant de $x$ et de $\lambda$

$$
\left|E_{x, \lambda}-\frac{c_{d}(x) \lambda^{d}}{(2 \pi)^{d}}\right| \leq C \lambda^{d-1}
$$

et donc (2.14) est conséquence de (2.5).

On en déduit en particulier le lemme suivant

Lemme 2.2. - Il existe $c_{2}>0$ tel que pour tout $x \in M$ et tout $\lambda>0$,

$$
P_{h}\left(u \in S_{h} ;|u(x)|>\lambda\right) \leq e^{-c_{2} \lambda^{2}} .
$$

$4^{\text {e }}$ SÉRIE - TOME $46-2013-$ NNo $^{\circ}$ 
En effet, d'après (2.12) et (2.13),

$$
P_{h}\left(u \in S_{h} ;|u(x)|>\lambda\right) \leq\left(1-\frac{\lambda^{2}}{\left|b_{x, h}\right|^{2}}\right)^{N_{h}-1} \leq e^{-\frac{N_{h}-1}{\left|b_{x, h}\right|^{2}} \lambda^{2}}=e^{-\frac{N_{h}-1}{e_{x, h}} \lambda^{2}}
$$

et le lemme 2.2 s'en déduit d'après le lemme 2.1.

Preuve du théorème 2. - On se limite encore ici au cas complexe (le cas réel étant similaire). Le théorème 2 est une conséquence des formules (2.12), (2.13) et de l'estimation (2.14). Remarquons que d'après les injections de Sobolev (1.3) il existe $0<c<C$ et $C_{1}>0$ tels que pour tout $h \in] 0,1]$ et tout $u \in S_{h}$ on a $c \leq\|u\|_{L^{\infty}} \leq C h^{-d / 2}$ et

$$
\left\|\nabla_{x} u\right\|_{L^{\infty}} \leq C_{1} h^{-(d / 2+1)} .
$$

Il en résulte

$$
\sup _{x}|u(x)| \leq \sup _{\alpha \in A}\left|u\left(x_{\alpha}\right)\right|+\varepsilon \Lambda
$$

dès que $\left(x_{\alpha}\right)_{\alpha \in A}$ est un réseau de points de $M$ de maille plus petite que $\varepsilon \Lambda h^{d / 2+1} / C_{1}$ (de cardinal de taille $C h^{-d d / 2+1}$ ). D'après (2.12), (2.13), le lemme 2.1, et $\left(1-t^{2}\right)^{N_{h}} \leq e^{-N_{h} t^{2}}$ pour $t \in[0,1]$, il existe $h_{0}>0$ tel que pour $\left.\left.h \in\right] 0, h_{0}\right]$ on ait

$$
\begin{aligned}
P\left(u \in S_{h},\|u\|_{L^{\infty}}>\Lambda\right) & \leq \sum_{\alpha \in A} P\left(\left|e v_{x_{\alpha}}\right|>(1-\varepsilon) \Lambda\right) \\
& \leq \sum_{\alpha \in A} \mathbf{1}_{(1-\varepsilon) \Lambda \leq\left|b_{x_{\alpha}, h}\right|}\left(1-(1-\varepsilon)^{2} \Lambda^{2} /\left|b_{x_{\alpha}, h}\right|^{2}\right)^{N_{h}-1} \\
& \leq \sum_{\alpha \in A} e^{-\left(N_{h}-1\right)(1-\varepsilon)^{2} \Lambda^{2} /\left|b_{x_{\alpha}, h}\right|^{2}} \\
& \leq \operatorname{card}(A) e^{-\operatorname{Vol}(M)(1-\varepsilon)^{2} \Lambda^{2} \frac{N_{h}-1}{N_{h}+C_{0} \operatorname{Vol}(M) h-d+1}} .
\end{aligned}
$$

On a $\liminf \inf _{h \rightarrow 0} \frac{N_{h}-1}{N_{h}+C_{0} \operatorname{Vol}(M) h^{-d+1}}>0$ d'après (2.7), et $\lim _{h \rightarrow 0} \frac{N_{h}-1}{N_{h}+C_{0} \operatorname{Vol}(M) h^{-d+1}}=1$ d'après (2.6) dans le cas $\lim _{h \rightarrow 0}\left(b_{h}-a_{h}\right) / h=+\infty$. Comme on a $\sharp(A) \leq C \varepsilon^{-d} \Lambda^{-d} h^{-d(d / 2+1)}$, (2.9) résulte de (2.19). La preuve du théorème 2 est complète.

\subsection{Mesures de défaut presque sûres}

On se place dans cette section sous l'hypothèse

$$
\lim _{h \rightarrow 0} \frac{b_{h}-a_{h}}{h}=+\infty,
$$

ce qui exclut le cas critique $b_{h}-a_{h} \sim D h$. On a alors d'après (2.6)

$$
\lim _{h \rightarrow 0} \frac{h^{-d+1}}{N_{h}}=0 \text {. }
$$

Lemme 2.3. - Soit $A(x, h D)$ un opérateur h-pseudodifférentiel classique sur $M$ de degré 0 et de symbole principal $a(x, \xi)$. Il existe $C_{0}>0$ tel que

$$
\left|\mathbb{E}_{h}((A(x, h D) u \mid u))-m_{a, h}\right| \leq C_{0} \frac{h^{-d+1}}{N_{h}},
$$


où on a noté

$$
m_{a, h}=\frac{\iint_{a_{h}<|\xi|_{x}<b_{h}} a(x, \xi) d x d \xi}{\iint_{a_{h}<|\xi|_{x}<b_{h}} d x d \xi}
$$

On remarquera que

$$
m_{a}=\lim _{h \rightarrow 0} m_{a, h}
$$

existe.

Démonstration. - Pour tout opérateur linéaire $A \operatorname{sur} E_{h}$ on a

$$
\mathbb{E}_{h}((A u \mid u))=\frac{\operatorname{tr}(A)}{N_{h}}
$$

et il suffit donc de vérifier qu'on a

$$
\left|\frac{\operatorname{tr}\left(\Pi_{h} A(x, h D) \Pi_{h}\right)}{N_{h}}-m_{a, h}\right| \leq C_{0} \frac{h^{-d+1}}{N_{h}} .
$$

Ce résultat est conséquence de travaux de Guillemin [17]. Ici, nous utiliserons la preuve donnée par Hörmander. On a d'après la preuve de [19, Theorem 29.1.7] (voir les pages 259260),

Proposition 2.4. - Notons $E_{\lambda}=1_{\sqrt{-\Delta}<\lambda}$ le projecteur spectral. Alors pour tout opérateur pseudodifférentiel d'ordre $0, B$, de symbole principal $b(x, \xi)$, on a

$$
\operatorname{Tr}\left(E_{\lambda} B E_{\lambda}\right)=\iint_{|\xi|_{x}<\lambda} b(x, \xi) d x d \xi+O\left(\lambda^{d-1}\right) .
$$

On remarque ensuite que dans (2.25), d'après la cyclicité de la trace, on peut remplacer $E_{\lambda} B E_{\lambda}$ par $E_{\lambda}^{2} B=E_{\lambda} B$. Par ailleurs (voir appendice B, en particulier la formule (B.1)), $B=A(x, h D)$ est, uniformément en $h \in] 0,1]$, un opérateur pseudodifférentiel d'ordre $0 \mathrm{au}$ sens de la proposition précédente. De plus, on peut réécrire (2.5) sous la forme

$$
N_{h}=\frac{h^{-d}}{(2 \pi)^{d}} \iint_{a_{h}<|\xi|_{x}<b_{h}} d x d \xi+O\left(h^{-d+1}\right) .
$$

On a alors

$$
\begin{aligned}
\operatorname{Tr}\left(\Pi_{h} A(x, h D) \Pi_{h}\right) & =\operatorname{Tr}\left(E_{h^{-1} b_{h}} A(x, h D)\right)-\operatorname{Tr}\left(E_{h^{-1} a_{h}} A(x, h D)\right) \\
& =\frac{1}{(2 \pi)^{d}} \iint_{h^{-1} a_{h}<|\xi|_{x}<h^{-1} b_{h}} a(x, h \xi) d x d \xi+O\left(h^{1-d}\right) \\
& =\frac{h^{-d}}{(2 \pi)^{d}} \iint_{a_{h}<|\xi|_{x}<b_{h}} a(x, \xi) d x d \xi+O\left(h^{1-d}\right) \\
& =m_{a, h}\left(N_{h}+O\left(h^{-d+1}\right)\right)+O\left(h^{-d+1}\right),
\end{aligned}
$$

ce qui avec $m_{a, h} \in O(1)$ implique (2.24). La preuve du lemme 2.3 est complète.

Soit $\left(h_{k}\right)_{k \geq 0}$ une suite de limite nulle et $X$ l'espace topologique produit $X=\Pi_{k} S_{h_{k}}$. On munit $X$ de la probabilité produit $\mathbb{P}=\Pi_{k} P_{h_{k}}$. 
THÉORÈme 3. - On suppose que les fonctions $a_{h}, b_{h}$ vérifient (2.20) et que la suite $\left(h_{k}\right)_{k \in \mathbb{N}}$ vérifie

$$
\begin{array}{r}
\exists L>0 ; \sum_{k} h_{k}^{L}<\infty \\
\lim _{k \rightarrow \infty} N_{h_{k}} /\left|\log \left(h_{k}\right)\right|=\infty
\end{array}
$$

(cette dernière hypothèse est toujours satisfaite en dimension $d \geq 2$ ). Alors on a, avec $m_{\sigma_{0}(A)}$ défini par (2.23),

$$
\mathbb{P}\left(\forall A \in \mathcal{E}_{h}^{0}, \lim _{k \rightarrow \infty}\left(A\left(x, h_{k} D\right) u_{k} \mid u_{k}\right)=m_{\sigma_{0}(A)}\right)=1 .
$$

REMARQUe 2.5. - Les hypothèses sur la suite $\left(h_{k}\right)$ sont évidemment satisfaites dans le cadre de la théorie de Littlewood-Paley pour laquelle on a $h_{k}=2^{-k}$. Le théorème 3 dit que pour presque toute suite $k \rightarrow u_{k} \in S_{h_{k}}$, la mesure de défaut semi-classique de la suite existe (i.e. on n'a pas besoin d'extraire une sous-suite) et est toujours égale à la mesure de Liouville normalisée sur la couronne $\left\{(x, \xi) \in T^{*} M,|\xi|_{x} \in I\right\}$.

Démonstration. - Comme il existe une partie dénombrable $\mathscr{D}$ de $\varepsilon_{h}^{0}$ telle que, pour tout $A \in \mathcal{E}_{h}^{0}$ et tout $\varepsilon>0$, il existe $B \in \mathscr{D}$ tel que

$$
\limsup _{h \rightarrow 0}\|A(x, h D)-B(x, h D)\|_{L^{2}}+\sup _{|\xi|_{x} \in I}\left|\sigma_{0}(A)(x, \xi)-\sigma_{0}(B)(x, \xi)\right| \leq \varepsilon,
$$

il suffit de prouver qu'on a pour tout $A \in \mathcal{E}_{h}^{0}$

$$
\mathbb{P}\left(\lim _{k \rightarrow \infty}\left(A\left(x, h_{k} D\right) u_{k} \mid u_{k}\right)=m_{\sigma_{0}(A)}\right)=1 .
$$

En écrivant $A=A_{1}+i A_{2}$ avec $A_{i}$ auto-adjoint, on a $\sigma_{0}(A)=\sigma_{0}\left(A_{1}\right)+i \sigma_{0}\left(A_{2}\right)$ et on peut donc aussi supposer que $A$ est auto-adjoint. Notons $f_{h}$ la variable aléatoire réelle sur $S_{h}, f_{h}(u)=(A(x, h D) u \mid u), d p_{h}$ sa loi, qui est à support dans $[-a, a]$ avec $a=\sup _{h}\|A(x, h D)\|_{L^{2}}<\infty$. Soit $\mathcal{M}_{h}$ la médiane de $f_{h}$. La fonction $f_{h}$ est lipschitzienne de constante de Lipschitz $\leq 2 \sup _{h}\|A(x, h D)\|_{L^{2}}$. D'après le théorème de concentration de la mesure de P. Levy (voir la proposition A.1 de l'appendice A). On remarquera que la sphère complexe $S_{h}$ s'identifie avec la sphère réelle $S^{2 N_{h}-1}$, et que les constantes sont donc cohérentes avec (A.7))

$$
P_{h}\left(u \in S_{h},\left|f_{h}(u)-\mathcal{M}_{h}\right| \geq r\right) \leq 2 \exp \left(-\frac{\left(N_{h}-1\right) r^{2}}{\left\|f_{h}\right\|_{\text {lips }}^{2}}\right)
$$

On a $\mathbb{E}\left(f_{h}\right)=\mathcal{M}_{h}+\int_{-a}^{a}\left(x-\mathcal{M}_{h}\right) d p_{h}$, donc d'après (2.31)

$$
\left|\mathbb{E}\left(f_{h}\right)-\mathcal{M}_{h}\right| \leq r+C \exp \left(-\frac{\left(N_{h}-1\right) r^{2}}{\left\|f_{h}\right\|_{\text {lips }}^{2}}\right) .
$$

Il existe donc $\alpha>0$ et $C_{1}>0$ tels que pour tout $\left.\left.r \in\right] 0,1\right]$ vérifiant $r \geq C_{1} \exp \left(-\alpha N_{h} r^{2}\right)$ on ait

$$
P_{h}\left(u \in S_{h},\left|f_{h}(u)-\mathbb{E}\left(f_{h}\right)\right| \geq 3 r\right) \leq 2 \exp \left(-a N_{h} r^{2}\right) .
$$

En utilisant (2.22), on en déduit qu'il existe $M_{0}$ tel que pour tout $M \geq M_{0}$, on a avec

$$
r_{h}=M N_{h}^{-1 / 2}|\log (h)|^{1 / 2}+C_{0} h^{-d+1} / N_{h}+o(1)_{h \rightarrow 0}
$$




$$
\left.P_{h}\left(u \in S_{h}, \mid f_{h}(u)-m_{\sigma_{0}(A)}\right) \mid \geq 4 r_{h}\right) \leq 2 \exp \left(-\alpha N_{h} r_{h}^{2}\right)
$$

et donc

$$
\left.\mathbb{P}\left(\sup _{l \geq k} \mid f_{h_{l}}(u)-m_{\sigma_{0}(A)}\right) \mid \leq 4 \sup _{l \geq k} r_{h_{l}}\right) \geq \Pi_{l \geq k}\left(1-2 \exp \left(-\alpha N_{h_{l}} r_{h_{l}}^{2}\right)\right) .
$$

On a d'après (2.34) et (2.28) $\lim _{l \rightarrow \infty} r_{h_{l}}=0$, et $\exp \left(-\alpha N_{h_{l}} r_{h_{l}}^{2}\right) \leq h_{l}^{\alpha M^{2}}$, donc d'après (2.27) (en choisissant $M$ tel que $\alpha M^{2} \geq L$ )

$$
\Pi_{l \geq k}\left(1-2 \exp \left(-\alpha N_{h_{l}} r_{h_{l}}^{2}\right) \geq 1-\varepsilon_{k}\right.
$$

avec $\lim _{k \rightarrow \infty} \varepsilon_{k}=0$. La preuve du théorème 3 est complète.

\subsection{Estimations $L^{q}$ presque sûres}

Dans cette section, on démontre que les normes $L^{q}$ sont bornées presque sûrement si $q<+\infty$. On calcule aussi l'ordre de grandeur des médianes des fonctions $\|u\|_{L^{q}}$ sur $S_{h}$ pour $2 \leq q \leq \infty$. On se limitera au cas $\mathbb{K}=\mathbb{C}$, les preuves dans le cas réel étant similaires.

Notons d'abord que les injections de Sobolev (1.3) peuvent être précisées en cas de localisation spectrale plus fine.

Proposition 2.6. - Il existe $C_{0}>0$ tel que pour tout $q \in[2, \infty]$ et tout $u \in E_{h}$ on ait

$$
\|u\|_{L^{q}(M)} \leq C_{0} N_{h}^{\left(\frac{1}{2}-\frac{1}{q}\right)}\|u\|_{L^{2}(M)} .
$$

En effet, pour $u \in E_{h}$, en utilisant (2.11), l'inégalité de Cauchy-Schwarz, ainsi que (2.15), on obtient

$$
|u(x)| \leq e_{x, h}^{1 / 2}\|u\|_{L^{2}} \leq C N_{h}^{1 / 2}\|u\|_{L^{2}}
$$

et la proposition résulte de $\|u\|_{L^{q}} \leq\|u\|_{L^{\infty}}^{1-2 / q}\|u\|_{L^{2}}^{2 / q}$ pour tout $q \in[2, \infty]$.

ThÉORÈme 4. - Il existe $c_{1}, c_{2}>0$ tels que pour tout $\left.q \in\right] 2,+\infty\left[\right.$, si on note $\mathcal{M}_{q, h}$ la médiane de la fonction $F(u)=\|u\|_{L^{q}}$ sur la sphère $\mathbb{S}_{h}$, on a pour tout $\Lambda \geq 0$, et tout $\left.\left.h \in\right] 0,1\right]$

$$
P_{h}\left(u \in S_{h} ;\left|\|u\|_{L^{q}}-\mathcal{M}_{q, h}\right|>\Lambda\right) \leq 2 e^{-c_{1} N_{h}^{\frac{2}{q}} \Lambda^{2}} .
$$

De plus, on a les estimations suivantes de la médiane

$$
1 \leq \mathcal{M}_{q, h} \leq c_{2} \sqrt{q} \quad \forall q \in[2, \infty[, \forall h \in] 0,1]
$$

Sous l'hypothèse $\lim _{h \rightarrow 0} N_{h}=\infty$, il existe $c_{3}>0, q_{0}>2$ et $\varepsilon_{0}>0$ tels que

$$
\left.\left.c_{3} \sqrt{q} \leq \mathcal{M}_{q, h} \quad \forall q \in\left[q_{0}, \varepsilon_{0} \log \left(N_{h}\right)\right], \forall h \in\right] 0,1\right] .
$$

Enfin, sous l'hypothèse $\lim _{h \rightarrow 0} \frac{b_{h}-a_{h}}{h}=\infty$, pour tout $\left.\gamma \in\right] 0,1\left[\right.$, il existe $q_{\gamma}>2$ et $\varepsilon_{\gamma}>0$ tels que

$$
\left.\left.\gamma \sqrt{\frac{q}{2 e \operatorname{Vol}(M)}} \leq \mathcal{M}_{q, h} \leq \frac{1}{\gamma} \sqrt{\frac{q}{2 e \operatorname{Vol}(M)}} \quad \forall q \in\left[q_{\gamma}, \varepsilon_{\gamma} \log \left(N_{h}\right)\right], \forall h \in\right] 0,1\right] .
$$


Démonstration. - D’après la proposition 2.6, on a

$$
\begin{aligned}
|F(u)-F(v)| & =\left|\|u\|_{L^{q}}-\|v\|_{L^{q}}\right| \\
& \leq\|u-v\|_{L^{q}} \leq C_{0} N_{h}^{\left(\frac{1}{2}-\frac{1}{q}\right)}\|u-v\|_{L^{2}} \leq C N_{h}^{\left(\frac{1}{2}-\frac{1}{q}\right)} \operatorname{dist}(u, v) .
\end{aligned}
$$

On en déduit

$$
\exists C>0 ; \forall 2 \leq p \leq+\infty,\|F\|_{\text {Lips }} \leq C N_{h}^{\left(\frac{1}{2}-\frac{1}{q}\right)},
$$

donc en utilisant le résultat de concentration de la mesure (voir appendice A, proposition A.1) à la fonction $F(u)=\|u\|_{L^{q}}$ sur la sphère $\mathbb{S}_{h}$ on obtient

$$
P_{h}\left(\left|F(u)-M_{q, h}\right|>r\right) \leq 2 e^{-\frac{N_{h}-1}{C^{2} N_{h}} N_{h}^{\frac{2}{q}} r^{2}}
$$

ce qui implique (2.39). Pour l'estimation (2.40) de la médiane, en utilisant

$$
\mathbb{E}_{h}\left(|g|^{q}\right)=q \int_{0}^{+\infty} \lambda^{q-1} P_{h}(|g|>\lambda) d \lambda
$$

on obtient, d'après (2.12) et (2.13),

$$
\begin{aligned}
\mathbb{E}_{h}\left(\|u\|_{L^{q}}^{q}\right) & =\int_{S_{h}} \int_{M}|u(x)|^{q} d x d p_{h} \\
& =\int_{M} \int_{S_{h}}|u(x)|^{q} d p_{h} d x \\
& =q \int_{M} \int_{0}^{\infty} \lambda^{q-1} P_{h}(|u(x)|>\lambda) d \lambda d x \\
& =q \int_{M} \int_{0}^{e_{x, h}^{1 / 2}} \lambda^{q-1}\left(1-\frac{\lambda^{2}}{e_{x, h}}\right)^{\left(N_{h}-1\right)} d \lambda d x \\
& =q\left(\int_{M} e_{x, h}^{q / 2}\right)\left(\int_{0}^{1} z^{q-1}\left(1-z^{2}\right)^{\left(N_{h}-1\right)} d z\right)=\left(\mathscr{G}_{q, h}\right)^{q} .
\end{aligned}
$$

Le lecteur pourra vérifier qu'on a bien $\mathscr{C}_{2, h}=1$. En notant $B(x, y)=\int_{0}^{1} t^{x-1}(1-t)^{y-1} d t$ la fonction béta, on a

$$
\int_{0}^{1} z^{q-1}\left(1-z^{2}\right)^{\left(N_{h}-1\right)} d z=\frac{1}{2} B\left(q / 2, N_{h}\right)=\frac{\Gamma(q / 2) \Gamma\left(N_{h}\right)}{2 \Gamma\left(q / 2+N_{h}\right)}
$$

donc, en utilisant le lemme 2.1 et la formule de Stirling,

$$
\left(\mathscr{G}_{q, h}\right)^{q} \leq q C^{q}\left(\frac{N_{h}}{N_{h}+\frac{q}{2}}\right)^{N_{h}+q / 2-1 / 2} \Gamma(q / 2) \quad \text { d'où } \quad \mathscr{C}_{q, h} \leq C^{\prime} \sqrt{q} .
$$

D'après l'inégalité de Bienaymé-Tchebychev, on a

$$
P_{h}\left(\|u\|_{L^{q}}>t\right) \leq \frac{1}{t^{q}} \mathbb{E}_{h}\left(\|u\|_{L^{q}}^{q}\right)=\left(\frac{\mathscr{C}_{q, h}}{t}\right)^{q}
$$

En choisissant $t=\mathcal{M}_{q, h}$, on obtient

$$
\frac{1}{2} \leq \lim _{\varepsilon \rightarrow 0} P_{h}\left(\|u\|_{L^{q}}>\mathcal{M}_{q, h}-\varepsilon\right) \leq\left(\frac{\mathscr{C}_{q, h}}{\mathcal{M}_{q, h}}\right)^{q}
$$

qui implique

$$
\mathcal{M}_{q, h} \leq 2^{1 / q} \mathscr{G}_{q, h}
$$


donc $\mathcal{M}_{q, h} \leq c_{2} \sqrt{q}$ d'après (2.48). Comme $F(u)=\|u\|_{L^{q}} \geq\|u\|_{L^{2}}=1$, on a aussi $\mathcal{M}_{q, h} \geq 1$, ce qui prouve (2.40).

Prouvons à présent (2.41). On remarque d'abord qu'on a, en utilisant $\lim _{h \rightarrow 0} N_{h}=\infty$, (2.46) et le lemme 2.1, avec $0<a_{1} \leq a_{2}$ indépendants de $h$ petit et de $q \in\left[2, \log \left(N_{h}\right)\right]$

$$
\mathscr{G}_{q, h} \in\left[a_{1} \sqrt{q}, a_{2} \sqrt{q}\right]
$$

et

$$
\begin{aligned}
\left|\mathscr{G}_{q, h}-\mathcal{M}_{q, h}\right|^{q} & =\left|\|F\|_{L^{q}\left(\mathbb{S}_{h}\right)}-\left\|\mathcal{M}_{q, h}\right\|_{L^{q}\left(\mathbb{S}_{h}\right)}\right|^{q} \\
& \leq\left\|F-\mathcal{M}_{q, h}\right\|_{L^{q}\left(\mathbb{S}_{h}\right)}^{q} \\
& =q \int_{0}^{+\infty} \lambda^{q-1} P_{h}\left(\left|F-\mathcal{M}_{q, h}\right|>\lambda\right) d \lambda \\
& \leq q \int_{0}^{+\infty} \lambda^{q-1} e^{-c_{1} N_{h}^{\frac{2}{q}} \lambda^{2}} d \lambda \\
& =\frac{q}{2 N_{h} c_{1}^{q / 2}} \Gamma(q / 2) .
\end{aligned}
$$

On en déduit

$$
\left|\mathscr{G}_{q, h}-\mathcal{M}_{q, h}\right| \leq \frac{C}{N_{h}^{1 / q}} \sqrt{q}
$$

qui implique (2.41) d'après (2.51). Montrons maintenant (2.42). On a d'après (2.14)

$$
\left(\int_{M} e_{x, h}^{q / 2} d x\right)^{1 / q}=\frac{N_{h}^{1 / 2}}{\operatorname{Vol}(M)^{1 / 2-1 / q}}\left(1+o_{h}(1)\right), \quad \lim _{h \rightarrow 0} o_{h}(1)=0
$$

et il en résulte en utilisant (2.46), pour $q \leq \log \left(N_{h}\right)$

$$
\mathscr{Q}_{q, h}=\sqrt{\frac{q}{2 e \operatorname{Vol}(M)}}\left(\left(1+o_{h, q}(1)\right)\right)
$$

avec $\lim _{q \rightarrow \infty, h \rightarrow 0} o_{h, q}(1)=0$. L'encadrement (2.42) résulte alors de (2.53). La preuve du théorème 4 est complète.

Nous allons à présent déduire du théorème 4 une estimation $L^{\infty}$ qui complète le théorème 2 .

ThÉorème 5. - On suppose qu'il existe a $>0$ tel que $N_{h} \geq h^{-a}$ pour $h$ petit (cette hypothèse est toujours vérifiée en dimension $d \geq 2$ sous l'hypothèse (2.2), avec $D$ assez grande pour pouvoir utiliser (2.7)). Il existe $h_{0}, c_{0}, c_{1}>0$ tels que pour tout $\left.\left.h \in\right] 0, h_{0}\right]$ on ait

$$
\mathbb{E}_{h}\left(\|u\|_{L^{\infty}}\right) \in\left[c_{0} \sqrt{|\log (h)|}, c_{1} \sqrt{|\log (h)|}\right]
$$

et en notant $\mathcal{M}_{\infty, h}$ la médiane de $F_{\infty}(u)=\|u\|_{L^{\infty}}$

$$
\mathcal{M}_{\infty, h} \in\left[c_{0} \sqrt{|\log (h)|}, c_{1} \sqrt{|\log (h)|}\right]
$$

et

$$
P_{h}\left\{u \in S_{h} ;\left|\|u\|_{L^{\infty}}-\mathcal{M}_{\infty, h}\right|>\lambda\right\} \leq C e^{-c \lambda^{2}} .
$$


Démonstration. - Il existe $C, C^{\prime}>0$ tels que pour tout $\left.\left.h \in\right] 0,1\right]$, tout $q>2$, et tout $u \in E_{h}$ on a d'après (1.4)

$$
\|u\|_{q} \leq C\|u\|_{L^{\infty}} \leq C^{\prime} h^{-d / q}\|u\|_{q}
$$

donc en choisissant $q_{h}=a \varepsilon_{0}|\log (h)|$, on obtient avec $C_{1}=C^{\prime} e^{d / a \varepsilon_{0}}$ indépendant de $h$

$$
\mathbb{E}_{h}\left(\|u\|_{q_{h}}\right) \leq \mathbb{E}_{h}\left(\|u\|_{L^{\infty}}\right) \leq C_{1} \mathbb{E}_{h}\left(\|u\|_{q_{h}}\right) .
$$

Or, pour tout $q \in[2, \infty]$, on a par le résultat de concentration de la mesure d'après (2.45) (qui s'applique aussi à $q=\infty$ ), avec $c_{1}$ indépendant de $h$

$$
\begin{aligned}
\left|\mathbb{E}_{h}\left(\|u\|_{q}\right)-\mathcal{M}_{q, h}\right| & \leq \int\left|\|u\|_{q}-\mathcal{M}_{q, h}\right| d P=\int_{0}^{\infty} P_{h}\left(\left|\|u\|_{q}-\mathcal{M}_{q, h}\right|>\lambda\right) d \lambda \\
& \leq 2 \int_{0}^{\infty} e^{-c_{1} \lambda^{2}}=\sqrt{\pi / c_{1}} .
\end{aligned}
$$

Comme on a $q_{h} \leq \varepsilon_{0} \log \left(N_{h}\right),(2.40)$ et (2.41) impliquent $\mathcal{M}_{q_{h}, h} \simeq \sqrt{q_{h}}$. On déduit alors de (2.60) pour $h$ petit $\mathbb{E}_{h}\left(\|u\|_{q_{h}}\right) \simeq \sqrt{q_{h}}$, donc aussi d'après (2.59), $\mathbb{E}_{h}\left(\|u\|_{L^{\infty}}\right) \simeq \sqrt{q_{h}}$, donc à nouveau par (2.60) $\mathcal{M}_{\infty, h} \simeq \sqrt{q_{h}}$. L'estimée (2.58) est maintenant conséquence de cette dernière estimation, du principe de concentration de la mesure (A.7) et de (2.44) La preuve du théorème 5 est complète.

\section{Application aux harmoniques sphériques}

Rappelons que les fonctions propres du laplacien sur la sphère $\mathbb{S}^{d}$ (muni de sa métrique standard) sont les harmoniques sphériques de degré $k$ (restrictions à la sphère $\mathbb{S}^{d}$ des polynômes harmoniques de degré $k$ ) et qu'elles vérifient :

- Pour tout $k \in \mathbb{N}$, l'espace vectoriel sur $\mathbb{K}$ des harmoniques sphériques de degré $k, E^{k}$, est de dimension

$$
N_{k}=\left(\begin{array}{c}
k+d \\
d
\end{array}\right)-\left(\begin{array}{c}
k+d-2 \\
d
\end{array}\right) \sim_{k \rightarrow+\infty} \frac{2}{(d-1) !} k^{d-1}
$$

- Pour tout $e \in E^{k},-\boldsymbol{\Delta}_{\mathbb{S}^{d}} e=k(k+d-1) e$.

- L'espace vectoriel engendré par les harmoniques sphériques est dense dans $L^{2}\left(\mathbb{S}^{d}\right)$.

On peut identifier l'espace des bases orthonormales de l'espace $E^{k}$ (muni de la norme $L^{2}$ ) avec le groupe unitaire, $U\left(N_{k}\right)$, si $\mathbb{K}=\mathbb{C}$ et avec le groupe orthogonal, $O\left(N_{k}\right)$, si $\mathbb{K}=\mathbb{R}$. On munit $U\left(N_{k}\right)$ (resp. $O\left(N_{k}\right)$ ) de sa mesure de Haar, $\nu_{k}$ (resp. $\widetilde{\nu}_{k}$ ). On peut alors identifier l'espace des bases hilbertiennes de $L^{2}(M ; \mathbb{K})$ formées d'harmoniques sphériques avec

$$
\mathscr{B}=\Pi_{k \in \mathbb{N}} U\left(N_{k}\right), \quad\left(\text { resp. } \widetilde{\mathcal{B}}=\Pi_{k \in \mathbb{N}} O\left(N_{k}\right)\right)
$$

qu'on munit de la mesure de probabilité produit

$$
\nu=\otimes_{k} \nu_{k},\left(\operatorname{resp} . \widetilde{\nu}=\otimes_{k} \widetilde{\nu}_{k}\right) .
$$

On a alors le résultat suivant 
ThÉORÈme 6. - Soit $d \geq 2$. Il existe $c>0$ et pour tout $2 \leq q<+\infty, C_{q}>0$ tels que

$$
\begin{aligned}
& \nu\left(\left\{B=\left(b_{k, l}\right)_{k \in \mathbb{N}, l=1, \cdots N_{k}} \in \mathscr{B} ; \exists k, l ;\left|\left\|b_{k, l}\right\|_{L^{q}\left(\mathbb{S}^{d}\right)}-\mathcal{M}_{q, k}\right|>r\right\}\right) \leq C_{q} e^{-c r^{2}} \\
& \widetilde{\nu}\left(\left\{B=\left(b_{k, l}\right)_{k \in \mathbb{N}, l=1, \cdots N_{k}} \in \widetilde{\mathcal{B}} ; \exists k, l ;||\left|b_{k, l} \|_{L^{q}\left(\mathbb{S}^{d}\right)}-\mathcal{M}_{q, k}\right|>r\right\}\right) \leq C_{q} e^{-c r^{2}}
\end{aligned}
$$

où la médiane (dans le cas complexe) $\mathcal{M}_{q, k}$ vérifie avec $C$ indépendant de $q, k$

$$
\begin{aligned}
\left|\mathscr{G}_{q, k}-\mathcal{M}_{q, k}\right| & \leq \frac{C}{N_{k}^{1 / q}} \sqrt{q} \\
\mathscr{G}_{q, k} & =\frac{N_{k}^{\frac{1}{2}}}{\left(\operatorname{Vol}\left(\mathbb{S}^{d}\right)\right)^{\frac{1}{2}-\frac{1}{q}}}\left(q \frac{\Gamma(q / 2) \Gamma\left(N_{k}\right)}{2 \Gamma\left(q / 2+N_{k}\right)}\right)^{\frac{1}{q}},
\end{aligned}
$$

où

$$
\mathscr{G}_{q, k}=\left(\frac{q \Gamma(q / 2)}{2}\right)^{\frac{1}{q}}\left(1+\Theta\left(e^{-q N_{k}^{-1}}\right)\right)=\left(\sqrt{\frac{q}{2 e}}+\Theta\left(q^{-1 / 2}\right)\left(1+\vartheta\left(e^{-q N_{k}^{-1}}\right)\right) .\right.
$$

Démonstration. - On se limite au cas complexe, $\mathbb{K}=\mathbb{C}$, le cas réel étant similaire. On commence par remarquer que, quitte à augmenter la constante $C_{q}$, on peut se contenter de prouver le théorème pour les bases orthonormales du sous-espace $\oplus_{k \geq 1} E^{k} \mathrm{de} L^{2}$. On prend $h_{k}=k^{-1}$. On remarque alors que

$$
\sqrt{k(k+d-1)}=h_{k}^{-1} \sqrt{1+(d-1) h_{k}}=h_{k}^{-1}+\frac{d-1}{2}+O\left(h_{k}\right) .
$$

On en déduit qu'avec

$$
a_{h}=1+h\left(\frac{d-1}{2}-\frac{1}{4}\right), \quad b_{h}=1+h\left(\frac{d-1}{2}+\frac{1}{4}\right)
$$

on a pour $h_{k}=k^{-1}, k \in \mathbb{N}^{*}, E_{h_{k}}=E^{k}$ pour $k$ assez grand. On remarquera que l'hypothèse (2.2) avec $D$ assez grand n'est plus vérifiée, mais cela est sans importance car on a une meilleure estimation pour $e_{x, h}$ que celle donnée par le lemme 2.1 :

Lemme 3.1. - Pour tout $x \in \mathbb{S}^{d}$ et tout $k \in \mathbb{N}$, on a

$$
e_{x, h_{k}}=\frac{N_{k}}{\operatorname{Vol}(M)} .
$$

En effet, le noyau du projecteur orthogonal sur l'espace $E^{k}$ est donné par

$$
K(x, y)=\sum_{j=1}^{N_{h_{k}}} e_{j, k}(x) \overline{e_{j, k}(y)}
$$

où $\left(e_{j, k}\right)_{j=1}^{N_{h_{k}}}$ sont une base orthornomale de $E^{k}$. L'espace $E^{k}$ étant invariant par l'action des rotations de la sphère, on en déduit que $K(x, y)=K(R x, R y)$ pour toute rotation $R$. La fonction $x \in \mathbb{S}^{d} \mapsto e_{x, h_{k}}$ est donc constante, d'intégrale $N_{k}$, d'où le lemme 3.1. On en déduit que les théorèmes 2 et 4 s'appliquent, avec d'après (2.46), (2.47) et (2.53)

$$
\mathscr{G}_{q, k}=\frac{N_{k}^{\frac{1}{2}}}{\left(\operatorname{Vol}\left(\mathbb{S}^{d}\right)\right)^{\frac{1}{2}-\frac{1}{q}}}\left(q \frac{\Gamma(q / 2) \Gamma\left(N_{k}\right)}{2 \Gamma\left(q / 2+N_{k}\right)}\right)^{\frac{1}{q}}, \quad\left|\mathscr{C}_{q, k}-\mathcal{M}_{q, k}\right| \leq \frac{C}{N_{k}^{1 / q}} \sqrt{q} .
$$

En particulier, le théorème 4 implique 
Proposition 3.2. - Il existe $c_{0}, c_{1}>0$ tels que, pour tout $k \geq 1$ et tout $q \geq 2$, on a (3.3)

$$
\nu_{k}\left(\left\{B=\left(b_{l}\right)_{l=1}^{N_{k}} \in U\left(N_{k}\right) ; \exists 1 \leq l \leq N_{k} ;||\left|b_{l} \|_{L^{q}}-\mathcal{M}_{q, k}\right|>\Lambda\right) \leq c_{0} e^{-c_{1} k^{\frac{2(d-1)}{q}} \Lambda^{2}} k^{d-1} .\right.
$$

En effet, l'application

$$
B=\left(b_{l}\right)_{l=1}^{N_{k}} \in U\left(N_{k}\right) \mapsto b_{1} \in S_{k}
$$

envoie la mesure $\nu_{k}$ sur la mesure $P_{h_{k}}$ et donc d'après le théorème 4 pour tout $1 \leq l_{0} \leq N_{k}$

$$
\nu_{k}\left(\left\{B=\left(b_{l}\right)_{l=1}^{N_{k}} \in U\left(N_{k}\right) ;\left|\left\|b_{l_{0}}\right\|_{L^{q}}-\mathcal{M}_{q, k}\right|>\Lambda\right) \leq 2 e^{-c_{1} k^{\frac{2(d-1)}{q}} \Lambda^{2}} .\right.
$$

On en déduit que

$\nu_{k}\left(\left\{B=\left(b_{l}\right)_{l=1}^{N_{k}} \in U\left(N_{k}\right) ; \exists l_{0} \in\left\{1, \ldots, N_{k}\right\} ;||\left|b_{l_{0}} \|_{L^{q}}-\mathcal{M}_{q, k}\right|>\Lambda\right) \leq 2 e^{-c_{1} k \frac{2(d-1)}{q} \Lambda^{2}} N_{k}\right.$, ce qui implique clairement (3.3).

On définit maintenant les ensembles

$$
F_{k, r}=\left\{B=\left(b_{k}\right) \in U\left(N_{h}\right) ; \forall l_{0} ;\left|\left\|b_{k, l_{0}}\right\|_{L^{q}}-\mathcal{M}_{q, k}\right| \leq r\right\}
$$

et

$$
F_{r}=\cap_{k \geq 1} F_{k, r}=\left\{B=\left(b_{k, l}\right) ; k \in \mathbb{N}^{*}, l=1, \cdots N_{k} ; \forall k_{0}, l_{0} ;\left|\left\|b_{k_{0}, l_{0}}\right\|_{L^{q}}-M_{q, k}\right| \leq r\right\} .
$$

On a pour $r \geq 1$

$$
\nu\left(F_{r}^{c}\right) \leq \sum_{k \geq 1} \nu_{k}\left(F_{k, r}^{c}\right) \leq \sum_{k \geq 1} c_{0} e^{-c_{1} k \frac{2(d-1)}{q}} r^{2} k^{d-1} \leq \sum_{k \geq 1} C_{q} e^{-c_{1} k^{\frac{2(d-1)}{q}} r^{2} / 2} \leq C_{q} e^{-c_{1} r^{2} / 2} .
$$

Quitte à augmenter $C_{q}$ on a aussi $1 \leq C_{q} e^{-c_{1} r^{2} / 2}$ pour tout $r \leq 1$. La preuve du théorème 6 est complète.

On démontre de la même façon (en remplaçant le théorème 4 par le théorème 2 ) le résultat suivant (on renvoie à [37] pour un résultat similaire sur $\mathbb{S}^{2}$, avec une estimée en $\log ^{2}(k)$ au lieu de $\left.\log ^{1 / 2}(k)\right)$.

THÉORÈME 7. $-\exists C, c, c_{0}>0, \forall r \geq 0$

$$
\begin{aligned}
& \nu\left(\left\{B=\left(b_{k, l}\right)_{k \in \mathbb{N}, l=1, \cdots N_{k}} \in \mathscr{B} ; \exists k, l ;\left\|b_{k, l}\right\|_{L^{\infty}\left(\mathbb{S}^{d}\right)}>c_{0} \log ^{1 / 2}(k)+r\right\}\right) \leq C e^{-c r^{2}}, \\
& \widetilde{\nu}\left(\left\{B=\left(b_{k, l}\right)_{k \in \mathbb{N}, l=1, \cdots N_{k}} \in \widetilde{\mathcal{B}} ; \exists k, l ;\left\|b_{k, l}\right\|_{L^{\infty}\left(\mathbb{S}^{d}\right)}>c_{0} \log ^{1 / 2}(k)+r\right\}\right) \leq C e^{-c r^{2}} .
\end{aligned}
$$

On déduit aussi du théorème 6 le résultat suivant.

THÉORÈme 8. - La probabilité qu'on puisse extraire d'une base orthonormale une sous-suite bornée en norme $L^{\infty}$ est nulle : pour toute constante $C>0$,

$$
\begin{aligned}
& \nu\left(\left\{B=\left(b_{k, l}\right)_{k \in \mathbb{N}, l=1, \cdots N_{k}} \in \mathscr{B} ; \liminf _{k \rightarrow+\infty} \inf _{l=1, \cdots, N_{k}}\left\|b_{k, l}\right\|_{L^{\infty}\left(\mathbb{S}^{d}\right)} \leq C\right\}\right)=0, \\
& \widetilde{\nu}\left(\left\{B=\left(b_{k, l}\right)_{k \in \mathbb{N}, l=1, \cdots N_{k}} \in \mathcal{B} ; \liminf _{k \rightarrow+\infty} \inf _{l=1, \cdots, N_{k}}\left\|b_{k, l}\right\|_{L^{\infty}\left(\mathbb{S}^{d}\right)} \leq C\right\}\right)=0 .
\end{aligned}
$$


Démonstration. - On se limite au cas complexe. Notons $A_{k, C}$ l'événement de $U\left(N_{k}\right)$

$$
A_{k, C}=\left\{B=\left(b_{k, l}\right), \exists l \in\left\{1, \cdots, N_{k}\right\}, \quad\left\|b_{k, l}\right\|_{L^{\infty}\left(\mathbb{S}^{d}\right)} \leq C\right\} .
$$

D'après le théorème 4 , il existe $k_{C}$ et $q=q_{C}$ tels qu'on ait à la fois $\operatorname{Vol}\left(\mathbb{S}^{d}\right)^{1 / q} \leq 2$ et $M_{q, k} \geq 3 C$ pour tout $k \geq k_{C}$. Comme on a $\|u\|_{L^{q}\left(\mathbb{S}^{d}\right)} \leq \operatorname{Vol}\left(\mathbb{S}^{d}\right)^{\frac{1}{q}}\|u\|_{L^{\infty}}$, il résulte de (2.39) qu'on a

$$
\nu_{k}\left(A_{k, C}\right) \leq 2 N_{k} e^{-c_{1} N_{k}^{2 / q} C^{2}} \quad \forall k \geq k_{C} .
$$

La série $\sum_{k} N_{k} e^{-c_{1} N_{k}^{2 / q} C^{2}}$ étant convergente, il en résulte $\lim _{L \rightarrow \infty} \nu\left(\cup_{k \geq L} A_{k, C}\right)=0$, donc $\nu\left(\cap_{L} \cup_{k \geq L} A_{k, C}\right)=0$. La preuve du théorème 8 est complète.

REMARQUE 3.3. - Les résultats de cette section restent vrais sur une variété riemannienne compacte quelconque, sous une forme plus faible. En effet, si on décompose $L^{2}(M)$ en somme directe

$$
L^{2}(M)=\oplus_{k} E_{h_{k}},
$$

où les espaces $E_{h_{k}}$ correspondent à un choix de $b_{h}-a_{h}=M h$, la même preuve donnera que presque toute base hilbertienne de $L^{2}(M)$ respectant cette décomposition aura toutes ses normes $L^{q}$ uniformément bornées. La principale différence avec le cas des sphères étant que les éléments de $E_{h_{k}}$ ne sont plus des fonctions propres exactes, mais des fonctions propres approchées:

$$
u \in E_{h_{k}} \Rightarrow \sqrt{-\boldsymbol{\Delta}} u=h_{k}^{-1} u+O(1)_{L^{2}(M)} .
$$

Sur les variétés de Zoll (où toutes les géodésiques sont périodiques), la répartition en paquets des fonctions propres (voir [5]) permet d'améliorer cette propriété

$$
u \in E_{h_{k}} \Rightarrow-\Delta u=\omega_{k}^{2} u+O(1)_{L^{2}(M)} .
$$

\subsection{Estimées sur les tores $\mathbb{T}^{d}$}

Notons, pour $k \in \mathbb{N}, E_{k}$ l'espace propre du laplacien sur le tore $\mathbb{T}^{d}$ associé à la valeur propre $k$, qu'on munit de la norme $L^{2}\left(\mathbb{S}^{d}\right)$ et $N_{k}$ sa dimension,

$$
N_{k}=\sharp J_{k}, \quad J_{k}=\left\{n \in \mathbb{Z}^{d} ;|n|^{2}=k\right\}
$$

qui est le nombre de présentation de l'entier $k$ en somme de $d$ carrés d'entiers. On note

$$
\mathfrak{S}_{d}=\left\{k \in \mathbb{N} ; N_{k} \geq 1\right\},
$$

le spectre du laplacien et, pour $k \in \mathfrak{S}_{d}$, on munit alors les sphères unités $\mathbb{S}_{k}$ des espaces $E_{k}$ de la probabilité uniforme, l'espace des suites d'éléments de $\mathbb{S}_{k}, k \in \mathfrak{S}_{d}$, de la probabilité produit, et l'espace des bases orthonormales formées de fonctions propres de la mesure de probabilité naturelle comme précédemment.

4 e SÉRIE - TOME $46-2013$ - No 6 
3.1.1. Preuve de (1.16). - La démonstration est certainement très classique (et au moins implicite dans [8]). Nous l'incluons ici pour être complets. On a clairement d'après la formule de Weyl

$$
\sum_{\frac{n}{2}<m \leq n} N_{m}=C n^{\frac{d}{2}}+O\left(n^{\frac{d-1}{2}}\right)
$$

On en déduit que, pour tout $d \geq 2$, il existe une suite $n_{p} \rightarrow+\infty$ telle que

$$
N_{n_{p}} \geq c n_{p}^{\frac{d-2}{2}}
$$

et en considérant la fonction propre du laplacien sur $\mathbb{T}^{d}$ (de norme $L^{2}$ égale à 1$)$

$$
u_{p}(x)=N_{n_{p}}^{-\frac{1}{2}} \sum_{N=\left(n_{1}, \ldots, n_{k}\right) \in E_{n_{p}}} e^{i N \cdot x}
$$

on voit d'après l'injection de Sobolev que

$$
c n_{p^{\frac{d-2}{4}}}^{\frac{k}{2}} \leq\left\|u_{p}\right\|_{L^{\infty}} \leq C n_{p}^{\frac{k}{2 r}}\left\|u_{p}\right\|_{L^{r}}
$$

Donc

$$
c n_{p}^{\frac{d-2}{4}-\frac{k}{2 r}} \leq C\left\|u_{p}\right\|_{L^{r}}
$$

ce qui est (1.16).

3.1.2. Bases orthonormales sur les tores $\mathbb{T}^{d}$. - Il est clair si on choisit la base orthonormale de $E_{k}$ donnée par

$$
B=\left\{e_{n}(x)=\frac{1}{\sqrt{\operatorname{Vol}\left(\mathbb{T}^{d}\right)}} e^{i n \cdot x}, n \in J_{k}\right\}
$$

qu'on a

$$
E_{k}(x)=\frac{1}{\operatorname{Vol}\left(\mathbb{T}^{d}\right)} \sum_{n \in J_{k}}\left|e^{i n \cdot x}\right|^{2}=\frac{1}{\operatorname{Vol}\left(\mathbb{T}^{d}\right)} \sum_{n \in J_{k}} 1=\frac{N_{k}}{\operatorname{Vol}\left(\mathbb{T}^{d}\right)}
$$

On en déduit que tous les résultats des sections 2.1 et 2.3 s'appliquent dans ce cadre. La seule différence par rapport au cas des sphères est que $\lim _{k \rightarrow+\infty} N_{k}=+\infty$ n'est vrai que pour $d \geq 5$ (et alors $N_{k} \geq c k^{\frac{d}{2}-1}$, voir Grosswald [16, Chapter 12]). La situation est donc un peu moins favorable et l'analogue des théorèmes 6 et 7 n'est donc vrai que si $d \geq 5$. En revanche, le résultat plus faible suivant est vrai en toute dimension $d \geq 2$ (et la démonstration est conséquence immédiate des résultats des sections 2.1,2.3) et de $\lim \sup _{k \rightarrow \infty} N_{k}=\infty$ dès que $d \geq 2$.

ThÉORÈme 9. - Considérons l'espace des suites $U=\left(u_{k}\right)_{k \in \mathfrak{S}_{d}} ; \forall k \in \mathfrak{S}_{d}, u_{k} \in \mathbb{S}_{k}$, muni de sa mesure de probabilité naturelle $\nu$. Alors

$$
\nu\left(\left\{U ; \exists C>0 ; \forall k,\left\|u_{k}\right\|_{L^{\infty}\left(\mathbb{T}^{d}\right)} \leq C\right\}\right)=0 .
$$




\section{Application à la stabilisation des ondes}

On s'intéresse dans cette section à l'étude de la décroissance de l'énergie pour les solutions de l'équation des ondes amorties sur la variété riemannienne compacte connexe $(M, g)$ :

$$
\left(\partial_{t}^{2}-\boldsymbol{\Delta}\right) u+2 a(x) \partial_{t} u=0,\left.\quad u\right|_{t=0}=u_{0} \in H^{1}(M),\left.\partial_{t} u\right|_{t=0}=u_{1} \in L^{2}(M)
$$

où $a \in C^{\infty}\left(M ;\left[0, \infty[)\right.\right.$ est non identiquement nulle. Pour $u=\left(u_{0}, u_{1}\right) \in H^{1}(M) \times L^{2}(M)$, l'énergie de $u$ est

$$
\mathcal{E}(u)=\frac{1}{2} \int_{M}\left(\left|\nabla_{x} u_{0}\right|^{2}+\left|u_{1}\right|^{2}\right) d_{g} x
$$

On a $\mathcal{E}(u)=0$ si et seulement si $u_{1}=0$ et $u_{0}(x)$ est une constante, et pour $u(t, x)$ solution de (4.1),

$$
\mathcal{E}\left(\left(u, \partial_{t} u\right)(t=0)\right)-\mathcal{E}\left(\left(u, \partial_{t} u\right)(t)\right)=\int_{0}^{t} \int_{M} 2 a(x)\left|\partial_{s} u(s, x)\right|^{2} d_{g} x
$$

On renvoie à [24] et [30] pour des résultats généraux sur la décroissance de l'énergie et la théorie spectrale de l'équation des ondes amorties, et à [2] pour des résultats numériques. Rappelons simplement ici que, dès que $a \not \equiv 0$, on a pour toute solution $u$ de (4.1)

$$
\lim _{t \rightarrow+\infty} \mathcal{E}(u(t, .))=0
$$

et que si le support de $a$ contrôle géométriquement la variété $M$, c'est-à-dire si toute géodésique rencontre l'ensemble $\{x \in M ; a(x)>0\}$, alors la décroissance de l'énergie est uniforme (donc exponentielle) :

$$
\exists C, \epsilon>0, \forall u=\left(u_{0}, u_{1}\right) \in H^{1}(M) \times L^{2}(M), \quad \mathcal{E}(u(t, .)) \leq C e^{-\epsilon t} \mathcal{E}(u(0, .)) .
$$

Nous allons étudier ici la décroissance de l'énergie d'un point de vue probabiliste, et uniformément par rapport à la fréquence. Soit $A_{a}$ l'opérateur non borné sur l'espace de Hilbert $H^{1}(M) \times L^{2}(M)$

$$
A_{a}=\left(\begin{array}{cc}
0 & \text { Id } \\
\boldsymbol{\Delta} & -2 a
\end{array}\right), D\left(A_{a}\right)=H^{2} \oplus H^{1} .
$$

On notera $U(t)=e^{t A_{a}}$, de sorte que la solution du système (4.1) avec données $\left(u_{0}, u_{1}\right)$ vérifie $\left(u(t,),. \partial_{t} u(t,).\right)=U(t)\left(u_{0}, u_{1}\right)$. On a, pour tout $t \in \mathbb{R}, U(t)(1,0)=(1,0)$, de sorte que $U(t)$ opère sur l'espace de Hilbert $H=H^{1}(M) / \mathbb{C} 1 \oplus L^{2}(M)$, et on considérera aussi $A_{a}$, donné par (4.6), comme opérateur non borné sur $H$. Ce passage au quotient est justifié par le fait que, bien que la quantité $\int_{M} u(t,.) d_{g} x$ ne soit pas préservée pour les solutions de (4.1), la moyenne de $u$ sur $M$ n'intervient pas dans le calcul de l'énergie. Le produit scalaire sur $H$ est défini par $(u \mid v)_{\mathscr{E}}=\frac{1}{2} \int_{M}\left(\nabla_{x} u_{0} \bar{\nabla}_{x} v_{0}+u_{1} \bar{v}_{1}\right) d_{g} x$ avec $u=\left(u_{0}, u_{1}\right)$, $v=\left(v_{0}, v_{1}\right)$ où $u_{0}, v_{0}$ sont définis modulo des constantes, et on a $\mathscr{E}(u)=(u \mid u)_{\mathscr{E}}$. On notera $\|u\|_{\mathscr{E}}=\sqrt{\mathscr{E}(u)}$. Pour $u=\left(u_{0}, u_{1}\right) \in H$, on identifiera $u_{0} \in H^{1}(M) / \mathbb{C} 1$ avec la fonction sur $M, \sum_{\omega_{n}>0}\left(\int_{M} u_{0} e_{n}(x) d_{g} x\right) e_{n}$, qui est indépendante du choix du représentant de $u_{0} \in H^{1}(M)$. 
On choisit ici $0<a_{h}=c<c^{\prime}=b_{h}$ et on rappelle que $\widetilde{E}_{h}$ est le $\mathbb{R}$-sous-espace vectoriel de $H$ engendré par les fonctions propres $e_{n}$ du laplacien sur $M$ avec $h \omega_{n} \in\left[c, c^{\prime}\right]$. Pour $u_{0}=\sum_{\omega_{n}>0} u_{0, n} e_{n}, u_{1}=\sum_{\omega_{n} \geq 0} u_{1, n} e_{n}$, on notera aussi $\widetilde{\Pi}_{h}$ l'opérateur borné de $H$ sur $\widetilde{E}_{h}$

$$
\widetilde{\Pi}_{h}(u)=\left(\sum_{h \omega_{n} \in I} u_{0, n} e_{n}, \sum_{h \omega_{n} \in I} u_{1, n} e_{n}\right)
$$

et $i_{h}$ l'injection de $\widetilde{E}_{h}$ dans $H$. Alors $\widetilde{\Pi}_{h}$ est le projecteur orthogonal sur $\widetilde{E}_{h}$ et $i_{h}$ est son adjoint. On prendra garde au fait que le sous-espace $\widetilde{E}_{h}$ de $H$ n'est pas invariant par le groupe d'évolution $U(t)$. Toutefois, la notion d'échelle est conservée uniformément en $t \geq 0$ par le groupe $U(t)$. Plus précisément, on montrera à la fin de ce paragraphe le théorème suivant (complètement déterministe) qui démontre que les solutions de l'équation des ondes amorties, la localisation dyadique en énergie est préservée par le flot uniformément par rapport $\grave{a} t \in(0,+\infty)$.

Théorème 10. - Soient $I=\left[c, c^{\prime}\right], 0<c<c^{\prime}$, et $r>0$ fixés. Pour tout entier $N \geq 1$, il existe une constante $C_{N}$ telle que, pour tout $0<h<h^{\prime}<1$ avec $c h^{\prime}-c^{\prime} h \geq r h$, on a

$$
\sup _{t \geq 0}\left\|\widetilde{\Pi}_{h} U(t) i_{h^{\prime}}\right\|_{\mathscr{E}}+\sup _{t \geq 0}\left\|\widetilde{\Pi}_{h^{\prime}} U(t) i_{h}\right\|_{\delta} \leq C_{N} h^{N} .
$$

\subsection{Amortissement presque sûr}

Soit $S^{*}(M)=\left\{(x, \xi) \in T^{*} M,\left|\xi_{x}\right|=1\right\}$ le fibré cotangent unitaire et $\phi(t, \rho)$ le flot géodésique sur $S^{*}(M)$. Soit $\underline{a}(t, \rho)$ la moyenne de la fonction $a$ à l'instant $t$ le long du flot

$$
\underline{a}(t, \rho)=\frac{1}{t} \int_{0}^{t} a(x(\phi(s, \rho))) d s .
$$

La mesure de Liouville canonique $d \mu$ sur $S^{*}(M)$ étant invariante par le flot géodésique, la fonction

$$
\operatorname{Bir}(\rho)=\lim _{t \rightarrow \infty} \underline{a}(t, \rho)
$$

existe $\mu$-presque partout d'après le théorème ergodique de Birkhoff.

On notera $\widetilde{S}_{h}$ la sphère unité de $\widetilde{E}_{h}$, et $\widetilde{P}_{h}$ la probabilité uniforme sur $\widetilde{S}_{h}$. Comme application simple des résultats de la section 2, on a le théorème suivant qui relie le comportement de la fonction de Birkhoff à la décroissance de l'énergie.

THÉORÈme 11. - On suppose que la fonction de Birkhoff vérifie

$$
\mu\left(\rho \in S^{*}(M), \operatorname{Bir}(\rho)=0\right)=0 .
$$

Alors pour tout $\varepsilon>0, \alpha \in] 0,1]$, il existe un temps $T>0$ tel que

$$
\forall h \in] 0,1], \widetilde{P}_{h}\left(u \in \widetilde{S}_{h}, \mathcal{E}(U(T) u)<\varepsilon\right) \geq 1-\alpha .
$$

Remarque 4.1. - Dans le cas particulier où le flot géodésique sur la variété $M$ est ergodique, on sait que, $\mu$ presque sûrement, la fonction de Birkhoff est constante et égale à la moyenne de la fonction a sur $M$ (donc strictement positive dès que la fonction a est non triviale). L'hypothèse (4.11) est donc dans ce cas toujours vérifiée. En revanche, dans ce cas l'hypothèse de contrôle géométrique n'est, elle, en général pas nécessairement vérifiée (on peut par exemple 
considérer le cas d'une surface compacte de courbure négative, où on prendrait la fonction a non triviale mais nulle au voisinage d'une trajectoire périodique).

RemaRque 4.2. - On remarquera que le temps $T$ à partir duquel on demande que l'énergie soit plus petite que $\varepsilon$ est indépendant de $h \in] 0,1]$, de sorte que l'estimation (4.12) est différente de la simple estimation (4.4). Dans le cas où $M$ est une sphère, et la fonction a identiquement nulle dans un voisinage de l'équateur, l'hypothèse (4.11) est violée. On laisse au lecteur le soin de prouver dans ce cas que l'estimation (4.12) est fausse pour $\alpha$ et $\varepsilon$ petits.

THÉORÈme 12. - Dans le cas où le flot est ergodique, ce qui implique que $\mu$ presque sûrement

$$
\operatorname{Bir}(\rho)=\frac{\int_{M} a(x) d x}{\operatorname{Vol}(M)}=\bar{a}>0
$$

et donc (4.11) est vérifiée, on suppose de plus l'estimée quantitative suivante sur le caractère exponentiellement mélangeant du flot :

$$
\forall \delta>0, \exists S(\delta)>0 ; \mu\left(\rho \in S^{*} M ;|\underline{a}(t, \rho)-\bar{a}|>\delta\right) \leq e^{-t S(\delta)} .
$$

Alors il existe $\mathcal{M}, \alpha, C, c, \epsilon>0$ tel que, pour tout $h \in] 0,1]$,

$$
P_{h}\left(\sup _{t \in[0, \epsilon \log (1 / h)]}\left(\mathcal{E}(U(t) u) e^{t \alpha}\right)>\mathcal{M}+r\right) \leq C e^{-c h^{-d+1} r^{2}} .
$$

Remarque 4.3. - L'hypothèse est vérifiée dans le cas où la variété $M$ est une surface de courbure négative constante et l'amortissement n'est pas trivial (voir Kifer [23] et Anantharaman [1, Theorem 3.1]).

Démonstration. - On commence par démontrer le théorème 11. Pour cela on va vérifier le lemme suivant :

Lemme 4.4. - On suppose que l'hypothèse (4.11) est satisfaite. Il existe $C, c>0$ et, pour tout $\varepsilon>0, T_{\varepsilon}$ et $h_{\varepsilon}$ tels qu'on ait pour tout $\left.\left.h \in\right] 0, h_{\varepsilon}\right]$

$$
\widetilde{P}_{h}\left(u \in \widetilde{S}_{h}, \mathcal{E}\left(U\left(T_{\varepsilon}\right) u\right)<\varepsilon\right) \geq 1-C e^{-c h^{-d} \varepsilon^{2}} .
$$

Démonstration. - Soit $\lambda=\sqrt{-\boldsymbol{\Delta}}$ et $j$ l'application de $H^{1}(M) \oplus L^{2}(M)$ dans $L^{2}(M) \oplus L^{2}(M)$ définie par

$$
j(u)=v \Longleftrightarrow v_{0}=\frac{\lambda u_{0}-i u_{1}}{\sqrt{2}}, v_{1}=\frac{\lambda u_{0}+i u_{1}}{\sqrt{2}} .
$$

On remarquera que $j$ est définie sur $H=H^{1}(M) / \mathbb{C} 1 \oplus L^{2}(M)$ et induit une isométrie de $\widetilde{E}_{h}$ sur $E_{h} \oplus E_{h}$. Posons $d_{ \pm}= \pm i \lambda-a, \mathscr{D}=\left(\begin{array}{cc}d_{+} & 0 \\ 0 & d_{-}\end{array}\right)$et $\mathcal{R}=\left(\begin{array}{ll}0 & a \\ a & 0\end{array}\right)$. Pour $u \in \widetilde{E}_{h}$, soit $v=j(u)$ et $v(t)=j(U(t) u)$. On vérifie aisément que $v(t)$ vérifie l'équation

$$
\partial_{t} v(t)=(\mathscr{D}+\mathscr{R}) v(t)
$$

Les opérateurs $d_{ \pm}$sont des opérateurs pseudo-différentiels classiques elliptiques de degré 1 , on a $d_{+}^{*}=d_{-}$(adjoint sur $L^{2}$ ) et comme $a \not \equiv 0$ ils sont bijectifs de $H^{s+1}(M)$ sur $H^{s}(M)$ pour tout $s \in \mathbb{R}$. Comme $\operatorname{Re}(\mathscr{D})=-a \mathrm{Id}$, on $\sup _{t \geq 0}\left\|e^{t \mathscr{D}}\right\|_{L^{2}} \leq 1$. De plus, comme 
$\mathscr{D}$ commute à $e^{t \mathscr{D}}$ et que pour $k$ entier la norme $\left\|\mathscr{D}^{k} v\right\|_{L^{2}}$ est équivalente à la norme $H^{k}$, pour tout $s \in \mathbb{R}$, il existe $C_{s}$ tel que

$$
\sup _{t \geq 0}\left\|e^{t \mathscr{D}}\right\|_{H^{s}(M)} \leq C_{s}
$$

Rappelons que la solution $v(t)$ de (4.17) avec $v(0)=v$ vérifie

$$
\begin{aligned}
v(t) & =e^{t \mathscr{D}} v+r(t, v), \\
\sup _{t \in[0, T]}\|r(t, v)\|_{L^{2}} & \leq C_{T}\|v\|_{H^{-1}} .
\end{aligned}
$$

La preuve de (4.19) est standard : on commence par vérifier que si $b_{-1}$ est un opérateur pseudo-différentiel de degré -1 et de symbole principal $a(x)|\xi|_{x}^{-1} / 2 i$, alors en posant $I=\mathrm{Id}+B_{-1}, B_{-1}=\left(\begin{array}{cc}0 & b_{-1} \\ -b_{-1} & 0\end{array}\right)$, on a $I(\mathscr{D}+\mathscr{R})=\mathscr{D} I+\widetilde{Q}_{-1}$ où $\widetilde{Q}_{-1}$ est une matrice $2 \times 2$ de pseudos de degré -1 . Comme on peut choisir $b_{-1}$ tel que $I$ soit inversible sur $H^{s}(M) \times H^{s}(M)$ pour tout $s$, la fonction $w(t)=I v(t)$ vérifie $\partial_{t} w(t)=\left(\mathscr{D}+Q_{-1}\right) w(t)$ avec $Q_{-1}=\widetilde{Q}_{-1} I^{-1}$, et la formule de Duhamel prouve qu'on a

$$
\sup _{t \in[0, T]}\left\|w(t)-e^{t \mathscr{D}} I v\right\|_{L^{2}} \leq C_{T}\|I v\|_{H^{-1}} .
$$

Or (4.18) (pour $s=0$ et $s=-1$ ) implique $\sup _{t \geq 0}\left\|e^{t \mathscr{D}} I v-I e^{t \mathscr{D}} v\right\|_{L^{2}} \leq C\|v\|_{H^{-1}}$, ce qui prouve (4.19).

On notera $P_{h}^{(2)}$ la probabilité uniforme sur la sphère unité

$$
S_{h}^{(2)}=\left\{\left(v_{0}, v_{1}\right),\left\|v_{0}\right\|_{L^{2}}^{2}+\left\|v_{1}\right\|_{L^{2}}^{2}=1\right\}
$$

de $E_{h} \oplus E_{h}$, et $\mathbb{E}_{h}^{(2)}(f)$ l'espérance d'une v.a sur $S_{h}^{(2)}$. Soit $f_{t}(v)=\mathcal{E}\left(U(t) j^{-1} v\right)$. Alors on a par construction

$$
\widetilde{P}_{h}\left(u \in \widetilde{S}_{h}, \mathcal{E}(U(t) u)<\varepsilon\right)=P_{h}^{(2)}\left(v \in S_{h}^{(2)}, f_{t}(v)<\varepsilon\right)
$$

et d'après (4.19)

$$
\sup _{v \in S_{h}^{(2)}, t \in[0, T]}\left|f_{t}(v)-\left\|e^{t \mathscr{D}} v\right\|_{L^{2}}^{2}\right| \leq C_{T} h
$$

avec $C_{T}$ indépendant de $\left.\left.h \in\right] 0,1\right]$. On a

$$
\left\|e^{t \mathscr{D}} v\right\|_{L^{2}}^{2}=\left(B_{+}(t) v_{0} \mid v_{0}\right)_{L^{2}}+\left(B_{-}(t) v_{1} \mid v_{1}\right)_{L^{2}}
$$

avec $B_{ \pm}(t)=e^{t d_{ \pm}^{*}} e^{t d_{ \pm}}$. D'après le théorème d'Egoroff (voir par exemple [40, Chapter 11]), les opérateurs $B_{ \pm}(t)$ sont des pseudos de degré 0 et de symbole principal homogène de degré 0

$$
\sigma_{0}\left(B_{ \pm}(t)\right)(\rho)=e^{-2 t \underline{a}(t, \rho)} .
$$

Comme dans la preuve du lemme 2.3, on obtient donc

$$
\sup _{t \in[0, T]}\left|\mathbb{E}_{h}^{(2)}\left(f_{t}\right)-\int_{S^{*}(M)} e^{-2 t \underline{a}(t, \rho)} d \mu(\rho)\right|=f_{T}(h), \lim _{h \rightarrow 0} f_{T}(h)=0 .
$$

Soit $m_{t, h}$ la médiane de la v.a $f_{t}$ sur $S_{h}^{(2)}$. Comme $f_{t}$ est (uniformément en $t \geq 0$ et $h \in] 0,1]$ ) lipschitzienne sur $S_{h}^{(2)}$, on obtient, comme dans la preuve du théorème 3 , qu'il existe $C, c>0$ tels que pour tout $r \in] 0,1]$ 


$$
\begin{aligned}
\left.\sup _{t \geq 0} P_{h}^{(2)}\left(\mid f_{t}(v)-m_{t, h}\right) \mid \geq r\right) \leq C e^{-c h^{-d} r^{2}} \\
\left.\quad \Rightarrow \sup _{t \geq 0}\left|\mathbb{E}_{h}^{(2)}\left(f_{t}\right)-m_{t, h}\right| \leq \int_{0}^{+\infty} P_{h}^{(2)}\left(\mid f_{t}(v)-m_{t, h}\right) \mid \geq r\right) d r \leq C h^{d / 2} .
\end{aligned}
$$

D'après l'hypothèse (4.11), on a

$$
\limsup _{t \rightarrow \infty} \int_{S^{*}(M)} e^{-2 t \underline{t}(t, \rho)} d \mu(\rho) \leq \int_{S^{*}(M)} \limsup _{t \rightarrow \infty} e^{-2 t \underline{a}(t, \rho)} d \mu(\rho)=0 .
$$

Soit alors $T_{\varepsilon}$ tel que $\int_{S^{*}(M)} e^{-2 T_{\varepsilon} \underline{a}\left(T_{\varepsilon}, \rho\right)} d \mu(\rho) \leq \varepsilon / 8$. Soit $h_{\varepsilon}$ tel qu'on ait à la fois $f_{T_{\epsilon}}\left(h_{\varepsilon}\right) \leq \varepsilon / 8$ et $C h_{\varepsilon}^{d / 2} \leq \varepsilon / 4$. Alors (4.22) et la deuxième ligne de (4.23) impliquent pour tout $\left.h \in] 0, h_{\varepsilon}\right], m_{t, h} \leq\left|\mathbb{E}_{h}^{(2)}\left(f_{t}\right)\right|+\left|\mathbb{E}_{h}^{(2)}\left(f_{t}\right)-m_{t, h}\right| \leq \varepsilon / 2$, et donc la première ligne de (4.23) implique (avec $r=\varepsilon / 2$ ) et pour tout $\left.h \in] 0, h_{\varepsilon}\right]$

$$
P_{h}^{(2)}\left(\left|f_{T_{\varepsilon}}(v)\right| \geq \varepsilon\right) \leq C e^{-c h^{-d} \varepsilon^{2} / 4} .
$$

La preuve du lemme 4.4 est complète.

La preuve du théorème 11 est une conséquence facile de l'estimation (4.15) : quitte à diminuer $h_{\varepsilon}$, on peut toujours supposer $C e^{-c h^{-d} \varepsilon^{2}} \leq \alpha$ pour $\left.\left.h \in\right] 0, h_{\varepsilon}\right]$. D'après (4.4), on a

$$
\lim _{t \rightarrow \infty} \inf _{h \in\left[h_{\varepsilon}, 1\right]} \widetilde{P}_{h}\left(u \in \widetilde{S}_{h}, \mathcal{E}(U(t) u)<\varepsilon\right)=1 .
$$

Soit alors $T \geq T_{\varepsilon}$ tel que pour tout $h \in\left[h_{\varepsilon}, 1\right]$ on ait $\widetilde{P}_{h}\left(u \in \widetilde{S}_{h}, \mathcal{E}(U(T) u)<\varepsilon\right) \geq 1-\alpha$. Comme l'énergie est décroissante, on a aussi d'après (4.15), pour tout $h \in] 0, h_{\varepsilon}$ ],

$$
\widetilde{P}_{h}\left(u \in \widetilde{S}_{h}, \mathcal{E}(U(T) u)<\varepsilon\right) \geq 1-\alpha,
$$

ce qui prouve le théorème 11 .

Pour démontrer le théorème 12 , nous allons revisiter la preuve précédente. On remarque d'abord que par un argument simple de semi-groupe, la norme $\|w(t)\|_{H^{-1}}$ est majorée par $C e^{C T}\|v\|_{H^{-1}}$ et donc la constante $C_{T}$ apparaissant dans (4.19), et donc aussi dans (4.21), peut être estimée par $C e^{C T}$. On remarque ensuite que le théorème d'Egoroff reste vrai pour des temps grands, mais plus petits que $\epsilon \log (1 / h)$ (c'est en effet conséquence de [21, théorème 2.3.6], et des estimations exponentielles triviales sur le comportement en grand temps du flot bicaractéristique). Le prix à payer pour cela est que les opérateurs $B_{ \pm}(t)$ sont des opérateurs pseudodifférentiels dont les symboles sont dans des classes (légèrement) exotiques : $S_{h, h^{-\alpha}, h^{-\alpha}, N}$ selon la terminologie de [21, Chapter 1], où $\alpha>0$ peut être choisi arbitrairement petit si $\epsilon>0$ est choisi petit. On en déduit qu'il existe $\delta>0$ tel que

$$
\sup _{t \in[0, \epsilon \log (1 / h)]}\left|\mathbb{E}_{h}^{(2)}\left(f_{t}\right)-\int_{S^{*}(M)} e^{-2 t \underline{t}(t, \rho)} d \mu(\rho)\right| \leq C e^{C \epsilon \log (1 / h)} h^{1-2 \alpha} \leq C h^{\delta}
$$

où, dans la dernière inégalité, on a choisi $\epsilon>0$ assez petit. On déduit maintenant de l'hypothèse qu'il existe $\sigma>0$ tel que

$$
\int_{S^{*}(M)} e^{-2 t \underline{a}(t, \rho)} d \mu(\rho) \leq e^{-2 t(\bar{a}-\beta)}+e^{-S(\beta) t} \leq 2 e^{-2 \sigma t}
$$


On définit la variable aléatoire $g$ sur $S_{h}^{(2)}$ par la relation

$$
g=\int_{0}^{\epsilon \log (1 / h)} f_{t} e^{\sigma t} d t
$$

D’après ce qui précède, la médiane, $\mathcal{M}$, de la fonction $g$ est inférieure ou égale à

$$
C \int_{0}^{\epsilon \log (1 / h)} e^{\sigma t}\left(e^{-2 \sigma t}+h^{\delta}\right) d t
$$

donc, quitte à diminuer encore $\epsilon>0$, cette médiane est bornée. De plus, la norme Lipschitz de la fonction $g$ est clairement bornée pour $\epsilon$ petit par

$$
C \int_{0}^{\epsilon \log (1 / h)} e^{\sigma t} d t \leq C h^{-1 / 2}
$$

on en déduit que

$$
P_{h}(g>\mathcal{M}+r) \leq C e^{-c h^{-d+1} r^{2}} .
$$

Finalement, on remarque que, comme l'énergie de la solution des ondes amorties est une fonction décroissante, on a l'implication

$$
g \leq A \Rightarrow \forall t \in[0, \epsilon \log (1 / h)], \quad \int_{0}^{t} f_{t} e^{\sigma s} d s \leq A \quad \Rightarrow \quad f_{t} \leq \frac{\sigma A}{e^{\sigma t}-1}
$$

ce qui termine la démonstration du théorème 12 .

Nous allons à présent utiliser les résultats précédents pour obtenir un taux de décroissance presque sûr pour des données dans l'espace d'énergie. On peut identifier les données initiales $\left(u_{0}, u_{1}\right) \in H^{1}(M) / \mathbb{C} \oplus L^{2}(M)$ à valeurs réelles avec $u=\lambda u_{0}+i u_{1} \in L^{2}(M ; \mathbb{C})$. On note $\mathcal{M}$ l'espace des mesures sur $L^{2}(M ; \mathbb{C})$ introduites dans l'appendice $\mathrm{C}$, associées à la décomposition $L^{2}(M)=\sum_{k} E_{k}$ où les $E_{k}$ sont les blocs dyadiques standards et avec $\left(\alpha_{k}\right) \in l^{2}$. Toute mesure $\mathbb{P} \in \mathcal{M}$ définit ainsi une mesure de probabilité sur $H^{1}(M) / \mathbb{C} \oplus$ $L^{2}(M)$. On a alors

THÉORÈme 13. - On suppose que la fonction de Birkhoff vérifie (4.11). On suppose aussi que les mesures $p_{k}$ vérifient $H_{\gamma}, \gamma>0$ (voir l'appendice $C$ ). Par exemple, on peut prendre

$$
d p_{k}=\sqrt{\frac{2}{\pi}} e^{-\frac{r^{2}}{2}} d r
$$

ou

$$
d p_{k}=\delta_{r=1}
$$

Il existe alors un taux de décroissance $f(t)>0$ qui tend vers 0 à l'infini, qui ne dépend pas du choix de la mesure $\mathbb{P} \in \mathcal{M}$, tel que

$$
\mathbb{P}\left(\left\{u \in H^{1}(M) / \mathbb{C} \oplus L^{2}(M) ; \exists T, \mathcal{E}(U(t) u) \leq f(t) \forall t \geq T\right\}\right)=1 .
$$

RemARQUe 4.5. - On remarque que le théorème 13 et les choix $d p_{k}=\delta_{r=1},\left(\alpha_{k}\right)=\delta_{k=k_{0}}$ impliquent le théorème 11. Nous allons en fait déduire le théorème 13 du théorème 11. Si on omet l'uniformité par rapport à la famille de mesures $\mathcal{M}$, le résultat est vrai sans hypothèse sur la fonction de Birkhoff. Il est alors juste conséquence de la convergence vers 0 de l'énergie pour toute donnée initiale. 
Démonstration. - D'après le théorème 11 , pour tout $j \in \mathbb{N}$, il existe $T_{j}$ tel

$$
\forall k, \widetilde{P}_{k}\left(u \in \widetilde{S}_{k} ; \mathcal{E}\left(U\left(T_{j}\right) u\right)>2^{-j}\right) \leq 2^{-j}
$$

On peut supposer $T_{j}<T_{j+1}$ et $\lim T_{j}=\infty$, et on pose

$$
f(t)=1 \text { pour } t<T_{0}, \quad f(t)=\sum_{j} 2^{-j / 2} 1_{t \in\left[T_{j}, T_{j+1}[\right.} \quad \text { pour } t \geq T_{0}
$$

On déduit de (4.29) en utilisant $\mathcal{E}\left(U\left(T_{j}\right) u\right) \leq 1$ pour $u \in \widetilde{S}_{k}$

$$
\int_{\widetilde{S}_{k}} \mathcal{E}\left(U\left(T_{j}\right) u\right) d P_{k} \leq 2^{-j}+2^{-j}
$$

En écrivant pour $u \in E_{k}, u=r \omega$, avec $\omega \in \widetilde{S}_{k}$, on obtient $\left(\operatorname{avec} C=2 \sup _{k} \int r^{2} d p_{k}<\infty\right.$ d'après l'hypothèse $\left(H_{\gamma}\right)$, voir l'appendice $\mathrm{C}$ )

$$
\mathbb{E}\left(\mathcal{E}\left(U\left(T_{j}\right) u\right)=\int_{0}^{\infty} r^{2}\left(\int_{\widetilde{S}_{k}} \mathcal{E}\left(U\left(T_{j}\right) u\right) d P_{k}\right) d q_{k} \leq 2^{1-j} \int_{0}^{\infty} r^{2} d q_{k} \leq C 2^{-j}\left|\alpha_{k}\right|^{2}\right.
$$

On remarque maintenant d'après le théorème 10 qu'il existe $C>0$ tel que pour tout $t \geq 0$ et tout $u$ d'énergie finie

$$
u=\sum_{k} u_{k}, u_{k} \in E_{k} \Rightarrow \mathcal{E}(U(t) u) \leq C \sum_{k} \mathcal{E}\left(U(t) u_{k}\right) .
$$

On en déduit d'après (4.31)

$$
\mathbb{E}\left(\mathcal{E}\left(U\left(T_{j}\right) u\right) \leq C \sum_{k} \mathbb{E}\left(\mathcal{E}\left(U\left(T_{j}\right) u_{k}\right) \leq C 2^{-j}\left\|\left(\alpha_{k}\right)\right\|_{\ell^{2}}^{2} .\right.\right.
$$

D'après l'inégalité de Bienaymé-Tchebitchev, on obtient

$$
\mathbb{P}\left(\left\{u \in H^{1}(M) / \mathbb{C} \oplus L^{2}(M) ; \mathcal{E}\left(U\left(T_{j}\right) u\right) \geq 2^{-j / 2}\right\}\right) \leq C 2^{-j / 2}\left\|\left(\alpha_{k}\right)\right\|_{\ell^{2}}^{2} .
$$

\section{Notons}

$$
A_{j}=\left\{u ; \mathcal{E}\left(U\left(T_{j}\right) u\right) \leq 2^{-j / 2}\right\}, A^{j}=\cap_{k \geq j} A_{k} .
$$

La suite $A^{j}$ est croissante et on a $\mathbb{P}\left(A^{j}\right) \geq\left(1-C\left\|\left(\alpha_{k}\right)\right\|_{\ell^{2}}^{2} \sum_{k \geq j} 2^{-k / 2}\right)=1-C^{\prime} 2^{-j / 2}$. Si $A=\cup A^{j}$, on a $\mathbb{P}(A)=1$ et, pour tout $u \in A$, il existe $C$ tel que pour tout $t$ on ait $\mathcal{E}(U(t) u) \leq C f(t)$. En effet, pour $u \in A^{j}$ on a en utilisant la décroissance de l'énergie

$$
\mathcal{E}(U(t) u) \leq f(t), \quad \forall t \geq T_{j} .
$$

Le théorème 13 est démontré.

$4^{\text {e }}$ SÉRIE - TOME $46-2013-$ No $^{\circ} 6$ 


\subsection{Conservation de la notion d'échelle}

Nous démontrons dans cette section le théorème 10. Des résultats similaires ont déjà été démontrés dans le cadre de perturbations singulières (conditions de Dirichlet à l'extérieur d'un obstacle [15, Proposition II.2.4]), mais en restant dans le cadre auto-adjoint. Ici le point nouveau vient du caractère non auto-adjoint de la perturbation $a \partial_{t}$.

Démonstration. - Soit $N \geq 1$ tel que $c-c^{\prime} / N \geq c / 2$. Pour $h^{\prime} \geq N h$, on a $c h^{\prime}-c^{\prime} h \geq$ $\left(c-c^{\prime} / N\right) h^{\prime} \geq h^{\prime} c / 2$ et pour $h^{\prime} \leq N h$, on a $c h^{\prime}-c^{\prime} h \geq h^{\prime} r / N$. Avec $r^{\prime}=\min (c / 2, r / N)$ on a donc toujours $c h^{\prime}-c^{\prime} h \geq r^{\prime} h^{\prime}$, donc $c / h-c^{\prime} / h^{\prime} \geq r^{\prime} / h$. On choisit $b^{\prime}, b, d$ tels que

$$
c-r^{\prime}<b^{\prime}<b<c, \quad \text { et } \quad c^{\prime}<d .
$$

Soient $\psi, \phi$ des fonctions réelles $C^{\infty}$ sur $\mathbb{R}$, à valeurs dans $[0,1]$, avec $\psi=1$ près de ]$\left.-\infty, c-r^{\prime}\right], \psi=0$ sur $\left[b^{\prime}, \infty\left[\right.\right.$ et $\phi=1$ près de $\left[c, c^{\prime}\right]$ et $\phi$ à support dans $[b, d]$. Les opérateurs $\phi(h \lambda), \psi(h \lambda)$ opèrent sur $H=H^{1}(M) / \mathbb{C} 1 \oplus L^{2}(M)$ par la formule $\phi(h \lambda)\left(e_{j}\right)=\phi\left(h \omega_{j}\right) e_{j}$, et ils commutent aux opérateurs $\widetilde{\Pi}_{h}, \widetilde{\Pi}_{h^{\prime}}, i_{h}, i_{h^{\prime}}$. Comme on a d'après (4.33) $\psi(h \lambda) i_{h^{\prime}}=i_{h^{\prime}}$, $\phi(h \lambda) i_{h}=i_{h}, \widetilde{\Pi}_{h^{\prime}} \psi(h \lambda)=\widetilde{\Pi}_{h^{\prime}}, \widetilde{\Pi}_{h} \phi(h \lambda)=\widetilde{\Pi}_{h}$, il suffit de vérifier que, pour tout $N$, il existe une constante $C_{N}$, telle que

$$
\sup _{t \geq 0}\|\phi(h \lambda) U(t) \psi(h \lambda)\|_{\delta}+\sup _{t \geq 0}\|\psi(h \lambda) U(t) \phi(h \lambda)\|_{\delta} \leq C_{N} h^{N} .
$$

Comme l'adjoint de $U(t)$ est $U^{*}(t)=e^{t A_{a}^{*}}$, avec

$$
A_{a}^{*}=-\left(\begin{array}{cc}
0 & \mathrm{Id} \\
\Delta & 2 a
\end{array}\right),
$$

on a $U^{*}(t)=e^{-t A_{-a}}$, donc $U^{*}(t)\left(u_{0}, u_{1}\right)=e^{t A_{a}}\left(u_{0},-u_{1}\right)$. Il suffit donc d'estimer le terme $\|\phi(h \lambda) U(t) \psi(h \lambda)\|_{\mathscr{E}}$.

Nous allons construire un opérateur $\Theta_{h}$, borné sur $H$ pour tout $\left.\left.h \in\right] 0,1\right]$ fixé, qui commute à $\mathrm{U}(\mathrm{t})$, et qui vérifie

$$
\begin{aligned}
\phi(h \lambda) & =\phi(h \lambda) \Theta_{h}+R_{1, h} \\
\Theta_{h} \psi(h \lambda) & =R_{2, h}
\end{aligned}
$$

et tel que, pour tout $N$, il existe une constante $C_{N}$, telle que

$$
\left\|R_{1, h}\right\|_{\mathscr{E}}+\left\|R_{2, h}\right\|_{\mathscr{E}} \leq C_{N} h^{N}
$$

On aura alors

$$
\begin{aligned}
\phi(h \lambda) U(t) \psi(h \lambda) & =\left(\phi(h \lambda) \Theta_{h}+R_{1, h}\right) U(t) \psi(h \lambda) \\
& =\phi(h \lambda) U(t) \Theta_{h} \psi(h \lambda)+R_{1, h} U(t) \psi(h \lambda) \\
& =\phi(h \lambda) U(t) R_{2, h}+R_{1, h} U(t) \psi(h \lambda)
\end{aligned}
$$

et comme $\|\phi(h \lambda) U(t)\|_{\mathscr{E}} \leq 1$ et $\|U(t) \psi(h \lambda)\|_{\mathscr{E}} \leq 1$, on obtiendra d'après (4.36)

$$
\sup _{t \geq 0}\|\phi(h \lambda) U(t) \psi(h \lambda)\|_{\mathscr{E}} \leq C_{N} h^{N} .
$$

On effectue maintenant une réduction semi-classique en posant $\tau\left(u_{0}, u_{1}\right)=\left(u_{0}, h u_{1}\right)$ et en introduisant la norme semi-classique

$$
\left\|\left(w_{0}, w_{1}\right)\right\|_{E_{h}}^{2}=\frac{1}{2} \int_{M}\left(\left|h \nabla_{x} w_{0}\right|^{2}+\left|w_{1}\right|^{2}\right) d_{g} x
$$


de sorte qu'on a $\|\tau(u)\|_{E_{h}}^{2}=h^{2}\|u\|_{\mathscr{E}}^{2}$, donc pour tout opérateur $R,\left\|\tau R \tau^{-1}\right\|_{E_{h}}=\|R\|_{\delta}$, et

$$
\tau U(t) \tau^{-1}=e^{i t B / h}
$$

avec

$$
B=-i h \tau A_{a} \tau^{-1}=\left(\begin{array}{cc}
0 & -i \\
-i h^{2} \Delta & 2 i h a
\end{array}\right) \text {. }
$$

On a pour $z \in \mathbb{C}$

$$
\operatorname{Im}((z-B) w \mid w)_{E_{h}}=\operatorname{Im}(z)\|w\|_{E_{h}}^{2}-2 h \int_{M} a\left|w_{1}\right|^{2} / 2 d_{g} x .
$$

Si $U_{h}$ est la bande $U_{h}=\left\{z \in \mathbb{C}, \operatorname{Im}(z) \in\left[0,2 h\|a\|_{L^{\infty}}\right]\right\}$, la résolvante $(z-B)^{-1}$ existe donc pour $z \in \mathbb{C} \backslash U_{h}$, et vérifie

$$
\left\|(z-B)^{-1}\right\|_{E_{h}} \leq \frac{1}{\operatorname{dist}\left(z, U_{h}\right)}
$$

On pose

$$
f_{\varepsilon}(z)=\frac{1}{\sqrt{2 \pi \varepsilon}} \int_{J} e^{-(z-x)^{2} / 2 \varepsilon} d x, \quad J=[-d,-b] \cup[b, d] .
$$

La fonction $f_{\varepsilon}(z)$ est holomorphe dans $\mathbb{C}$ et vérifie, pour tout $z \in \mathbb{C},\left|f_{\varepsilon}(z)\right| \leq e^{\operatorname{Im}(z)^{2} / 2 \varepsilon}$. De plus,on a pour tout $z \in \mathbb{C}$

$$
\left|f_{\varepsilon}(z)\right| \leq \frac{C}{\sqrt{\varepsilon}} e^{\operatorname{Im}(z)^{2} / 2 \varepsilon-\operatorname{dist}(\operatorname{Re}(z), J)^{2} / 2 \varepsilon} .
$$

On définit alors l'opérateur $\Theta_{h}$ par

$$
\Theta_{h}=\frac{1}{2 i \pi} \int_{\gamma} f_{\varepsilon}(z)(z-B)^{-1} d z .
$$

Dans (4.46) on choisit le contour $\gamma$ de la forme $\gamma=\gamma_{-} \cup \gamma_{+} \cdot \gamma_{+}$est la réunion du segment $\left[-r_{0}, r_{0}\right]+i r_{+}, r_{+}=2 h\|a\|_{L^{\infty}}+\sqrt{\varepsilon}$, et des deux demi-droites $r_{0}+i r_{+}+\rho e^{i \theta_{0}}, \rho \geq 0$, et $-r_{0}+i r_{+}-\rho e^{-i \theta_{0}}, \rho \geq 0$, avec $\theta_{0}>0$ petit et $r_{0}>d$ grand; on oriente $\gamma_{+}$de droite à gauche. $\gamma_{-}$est la réunion du segment $\left[-r_{0}, r_{0}\right]-i \sqrt{\varepsilon}$, et des deux demi-droites $r_{0}-i \sqrt{\varepsilon}+\rho e^{-i \theta_{0}}, \rho \geq 0$, et $-r_{0}-i \sqrt{\varepsilon}-\rho e^{i \theta_{0}}, \rho \geq 0$; on oriente $\gamma_{-}$de gauche à droite. On choisit le paramètre $\varepsilon$ sous la forme $\varepsilon=h^{\nu}$ avec $\left.\left.\nu \in\right] 0,1 / 4\right]$. On a alors d'après (4.43) et (4.45), pour une constante $C$ indépendante de $h$

$$
\left\|\Theta_{h}\right\|_{E_{h}} \leq C \varepsilon^{-1}
$$

et $\Theta_{h}$ commute à $e^{i t B / h}$. On va construire des opérateurs $Q_{h, k}(z), k \geq 0$, et $R_{h, N}(z), N \geq 1$, holomorphes en $z$ près de $\gamma$, et qui vérifient pour tout $N \geq 1$ avec $C_{N}$ indépendant de $h, z$

$$
\begin{aligned}
(z-B) \sum_{k=0}^{N-1}\left(\frac{h}{i}\right)^{k} Q_{h, k}(z) & =\mathrm{Id}-h^{N} R_{h, N}(z), \\
\left\|R_{h, N}(z)\right\|_{E_{h}} & \leq C_{N} \varepsilon^{-(1+2 N)} .
\end{aligned}
$$


Posons $\Theta_{h, k}=\frac{1}{2 i \pi} \int_{\gamma} f_{\varepsilon}(z) Q_{h, k}(z) d z$ et $\Theta_{h}^{N}=\sum_{k=0}^{N-1}\left(\frac{h}{i}\right) \Theta_{h, k}$. On a pour tout $N \geq 1$ d'après (4.43), (4.45), (4.46) et (4.48), en utilisant $\varepsilon=h^{\nu}$

$$
\begin{aligned}
\Theta_{h} & =\Theta_{h}^{N}+\frac{h^{N}}{2 i \pi} \int_{\gamma} f_{\varepsilon}(z)(z-B)^{-1} R_{h, N}(z) d z, \\
\left\|\frac{h^{N}}{2 i \pi} \int_{\gamma} f_{\varepsilon}(z)(z-B)^{-1} R_{h, N}(z) d z\right\|_{E_{h}} & \leq C_{N}^{\prime} h^{N(1-2 \nu)-2 \nu} .
\end{aligned}
$$

Comme on a $\nu<1 / 2$, pour obtenir (4.35) et (4.36), il suffit de prouver que, pour tout $N_{2}$, il existe $N_{1} \geq N_{2}$ et $C$ indépendant de $h$ tels que

$$
\begin{aligned}
\phi(h \lambda) & =\phi(h \lambda) \Theta_{h}^{N_{1}}+R_{1, h, N_{1}}, \\
\Theta_{h}^{N_{1}} \psi(h \lambda) & =R_{2, h, N_{1}}, \\
\left\|R_{1, h, N_{1}}\right\|_{E_{h}}+\left\|R_{2, h, N_{1}}\right\|_{E_{h}} & \leq C h^{N_{2}} .
\end{aligned}
$$

Nous allons construire les opérateurs $Q_{h, k}(z)$ et $R_{h, N}(z)$ en utilisant le calcul $h$-pseudodifférentiel classique (voir [27]). Pour $m$ entier relatif, on notera $\mathcal{M}^{m}$ l'espace des opérateurs matriciels $2 \times 2$

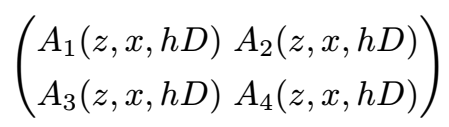

où $A_{j}(z, x, h D)$ est un polynôme de $z$ de degré $\leq m+p_{j}$, avec $p_{1}=p_{4}=0, p_{2}=-1$, $p_{3}=1$, et $A_{j}(z, x, h D)=\sum_{l=0}^{l=m+p_{j}} z^{l} A_{j, l}(x, h D)$ avec $A_{j, l} \in \mathcal{E}_{\mathrm{cl}}^{m-l+p_{j}}$. Par convention, un polynôme de degré strictement négatif est nul, de sorte que $\mathcal{M}^{m}$ est réduit à $\{0\}$ pour $m \leq-2$, et les éléments de $\mathcal{M}^{-1}$ sont de la forme $\left(\begin{array}{cc}0 & 0 \\ A(x, h D) & 0\end{array}\right)$ avec $A(x, h D) \in \mathcal{E}_{\mathrm{cl}}^{0}$. On a, pour tout $m, m^{\prime} \mathcal{M}^{m} \mathcal{M}^{m^{\prime}} \subset \mathcal{M}^{m+m^{\prime}}$ et pour $m \leq m^{\prime} \mathcal{M}^{m} \subset \mathcal{M}^{m^{\prime}}$. Dans une carte locale, on notera $\phi^{m}$ les symboles complets des éléments de $\mathcal{M}^{m}$. On a $z \operatorname{Id} \in \mathcal{M}^{1}$ et d'après (4.41), $B \in \mathcal{M}^{1}$. On posera $\beta_{1}(x, \xi)=\left(\begin{array}{cc}0 & -i \\ i|\xi|_{x}^{2} & 0\end{array}\right) \in \phi^{1}$, et $\delta=z^{2}-|\xi|_{x}^{2}$ de sorte que

$$
\left(z-\beta_{1}\right)^{-1}=\frac{1}{\delta}\left(\begin{array}{cc}
z & -i \\
-i|\xi|_{x}^{2} & z
\end{array}\right) \in \frac{1}{\delta} \phi^{1} .
$$

Soit $V_{l}$ un recouvrement fini de $M$ par des ouverts de cartes, $\theta_{l}, \theta_{l}^{\prime} \in C_{0}^{\infty}\left(V_{l}\right)$ avec $\theta_{l}^{\prime}$ égal à $1 \mathrm{au}$ voisinage du support de $\theta_{l}$ et $\sum_{l} \theta_{l}=1$. Dans chaque ouvert de carte $V_{l}$ on choisit des coordonnées $\left(x_{1}, \ldots, x_{d}\right) \in \mathbb{R}^{d}$, et on définit les opérateurs $Q_{h, k}(z)$ par la formule

$$
Q_{h, k}(z)=\sum_{l} \theta_{l}^{\prime} \mathrm{Op}\left(q_{k}(z, x, \xi)\right) \theta_{l}
$$

où les $q_{k}(z, x, \xi)$ sont définies par les formules de récurrence qui définissent l'inverse formel de $(z-B)$, soit

$$
\begin{aligned}
& q_{0}(z, x, \xi)=\left(z-\beta_{1}\right)^{-1} \\
& q_{n}(z, x, \xi)=\left(z-\beta_{1}\right)^{-1}\left(\sum_{|\alpha|+k=n,|\alpha| \geq 1} \frac{1}{\alpha !} \partial_{\xi}^{\alpha} \beta_{1} \partial_{x}^{\alpha} q_{k}+\sum_{|\alpha|+k=n-1} \frac{1}{\alpha !} \partial_{\xi}^{\alpha} \beta_{0} \partial_{x}^{\alpha} q_{k}\right)
\end{aligned}
$$


où $\beta_{1}+h \beta_{0} / i$ est le symbole complet de $B$ dans la carte locale, avec $\beta_{0} \in \phi^{0}$. D'après (4.41) on a pour tout $\alpha, \partial_{\xi}^{\alpha} \beta_{1} \in \phi^{1-|\alpha|}, \partial_{\xi}^{\alpha} \beta_{1}=0$ pour $|\alpha|>2$ et $\partial_{\xi}^{\alpha} \beta_{0} \in \phi^{-|\alpha|}, \partial_{\xi}^{\alpha} \beta_{0}=0$ pour $|\alpha| \geq 1$. De plus, on a pour $k \geq 0$ et tout $\alpha, \partial_{x}^{\alpha}\left(\delta^{-(1+2 k)} \phi^{1+3 k}\right) \subset \delta^{-(1+2 k+|\alpha|)} \phi^{1+3 k+2|\alpha|}$. On montre alors facilement par récurrence qu'on a pour tout $n \geq 0$

$$
q_{n}=\frac{p_{n}}{\delta^{1+2 n}}, \quad p_{n} \in \phi^{1+3 n} .
$$

L'opérateur $R_{h, N}(z)$ est alors défini par la formule (4.48) et a donc pour symbole

$$
-r_{h, N}(z, x, \xi)=\sum_{|\alpha|+k=N} \frac{1}{\alpha !} \partial_{\xi}^{\alpha} \beta_{1} \partial_{x}^{\alpha} q_{k}+\beta_{0} q_{N-1} \in \delta^{-(1+2 N)} \phi^{2+3 N}
$$

Comme on $\min _{z \in \gamma, x, \xi}|\delta| \simeq \varepsilon$ et que $|\delta| \simeq|z|^{2}+|\xi|_{x}^{2}$ pour $(z, \xi) \rightarrow \infty$, la deuxième ligne de (4.48) résulte de (4.55). Comme tous les $q_{n}$ sont des fractions rationnelles de $z$, on peut d'après (4.45) utiliser la formule des résidus pour calculer les symboles des opérateurs $\Theta_{h, k}$. Pour $\xi$ près de 0 on obtient, en utilisant la majoration (4.45), $\Theta_{h, k}(x, \xi) \in \Theta\left(e^{-c / h^{\nu}}\right)$ et pour $\xi \neq 0$ on trouve

$$
\Theta_{h, k}(x, \xi)=\frac{1}{(2 k) !} \partial_{z=|\xi|_{x}}^{2 k}\left(f_{\varepsilon}(z) \frac{p_{k}(z, x, \xi)}{\left(z+|\xi|_{x}\right)^{1+2 k}}\right)+\frac{1}{(2 k) !} \partial_{z=-|\xi|_{x}}^{2 k}\left(f_{\varepsilon}(z) \frac{p_{k}(z, x, \xi)}{\left(z-|\xi|_{x}\right)^{1+2 k}}\right) .
$$

Il résulte alors de (4.44) et (4.45) qu'on a $\Theta_{h}^{N}(x, \xi) \in \Theta\left(e^{-c / h^{\nu}}\right)$ pour $\xi$ grand, et pour $\xi$ borné

$$
\left|\partial_{x}^{\alpha} \partial_{\xi}^{\beta} \Theta_{h}^{N}(x, \xi)\right| \leq C_{\alpha, \beta} \varepsilon^{-(|\alpha|+|\beta|)}=C_{\alpha, \beta} h^{-\nu(|\alpha|+|\beta|)},
$$

de sorte que les opérateurs $\Theta_{h}^{N}(x, \xi)$ appartiennent à une classe admissible pour le calcul symbolique. Comme on a $f_{\varepsilon}(z)=1+\Theta\left(e^{-c / h^{\nu}}\right)$ au voisinage de $\left[-c^{\prime},-c\right] \cup\left[c, c^{\prime}\right]$ on déduit du fait que $\sum_{k}\left(\frac{h}{i}\right)^{k} Q_{h, k}(z, x, \xi)$ est le symbole de la résolvante $(z-B)^{-1}$ qu'on a, pour $|\xi|_{x}$ proche de $\left[c, c^{\prime}\right], \Theta_{h}(x, \xi)=1+\Theta\left(e^{-c / h^{\nu}}\right)$ et $\Theta_{h, k}(x, \xi)=\Theta\left(e^{-c / h^{\nu}}\right)$ pour $k \geq 1$. On a aussi $f_{\varepsilon}(z)=\Theta\left(e^{-c / h^{\nu}}\right)$ au voisinage de $\left[r^{\prime}-c, c-r^{\prime}\right]$. En choisissant $b$ proche de $c$ et $d$ proche de $c^{\prime}$, on obtient donc les formules (4.50) par les règles de calcul symbolique. Ceci termine la preuve du théorème 10 .

\section{Application aux ondes non-linéaires sur-critiques}

On considère dans cette section l'équation des ondes défocalisante sur une variété compacte $M$ (sans bord) de dimension 3 , pour une nonlinéarité polynomiale $u^{p}, p$ impair.

$$
\left(\partial_{t}^{2}-\boldsymbol{\Delta}\right) u+u^{p}=0,\left.u\right|_{t=0}=u_{0},\left.\partial_{t} u\right|_{t=0}=u_{1}, \quad\left(u_{0}, u_{1}\right) \in \mathcal{H}^{1}(M)=H^{1}(M) \times L^{2}(M) .
$$

On supposera $p \geq 7$, de sorte que l'équation (5.1) est sur-critique. On supposera aussi $u$ à valeurs réelles. Les mêmes énoncés restent vrais avec $u$ à valeurs complexes en remplaçant $u^{p}$ $\operatorname{par}|u|^{p-1} u$. Il est bien connu que l'équation précédente possède pour toutes données initiales dans $\left(H^{1}(M) \cap L^{p+1}(M)\right) \times L^{2}(M)$ des solutions faibles définies pour $t \in[0, \infty$ [, qui vérifient l'équation au sens des distributions, et telles que de plus

$$
\mathcal{E}(u)(t)=\int_{M} \frac{|\nabla u|^{2}}{2}+\frac{\left|\partial_{t} u\right|^{2}}{2}+\frac{u^{p+1}}{p+1} d x \leq \mathcal{E}(u)(0) .
$$

4 e SÉRIE - TOME $46-2013$ - No 6 
L'objet de ce paragraphe est de construire « beaucoup » de données initiales dans $H^{1} \times L^{2}$ pour lesquelles d'une part on sait construire une solution forte de l'équation (5.1) (sur un petit intervalle de temps $(0, T)$ ), et d'autre part, on sait que toutes les solutions faibles coïncident avec cette solution forte sur cet intervalle. Dans cette section, les mesures de probabilités $\mathcal{M}_{s}$ sont celles construites dans l'appendice $\mathrm{C}$ avec le choix $a_{k}=2^{k}$ de la théorie de Littlewood-Paley standard.

ThÉORÈme 14 (Existence). - Pour tout $p<+\infty$, et pour toute mesure de probabilités sur $\mathscr{D}^{\prime}(M)^{2}, \mathbb{P} \in \mathcal{M}_{1} \times \mathcal{M}_{0}$, pour $\mathbb{P}$-presque toute donnée initiale $\left(u_{0}, u_{1}\right) \in H^{1}(M) \times L^{2}(M)$, il existe $T>0$ et une solution forte de (5.1) dans l'espace affine

$$
\left(\cos (t \sqrt{-\boldsymbol{\Delta}}) u_{0}, \frac{\sin (t \sqrt{-\boldsymbol{\Delta}})}{\sqrt{-\boldsymbol{\Delta}}} u_{1}\right)+C^{0}\left((0, T) ; H^{2}(M)\right) \cap C^{1}\left((0, T) ; H^{1}(M) .\right.
$$

Cette solution vérifie l'identité d'énergie

$$
\mathcal{E}(u)(t)=\mathcal{E}(u)(0)
$$

et les estimées de Strichartz pour tout $r<+\infty$,

$$
\|u\|_{L^{\infty}((0, T) \times M)}+\|u\|_{L^{2}\left((0, T) ; W^{1, r}(M)\right)}+\left\|\partial_{t} u\right\|_{L^{2}\left((0, T) ; L^{r}(M)\right)}<+\infty .
$$

De plus on a la borne inférieure suivante sur le temps maximal d'existence $T_{\max }\left(u_{0}, u_{1}\right)$ :

$$
\exists \delta>0, C>0, c>0 ; \mathbb{P}\left(\left\{\left(u_{0}, u_{1}\right) \in \mathcal{H}^{1}(M) ; T_{\max }\left(u_{0}, u_{1}\right)<\lambda\right\}\right) \leq C e^{-c \lambda^{-\delta}} .
$$

Démonstration. - On suit la stratégie de [12], en y incorporant nos nouvelles estimations probabilistes. On cherche la solution sous la forme

avec

$$
u=\left(\cos (t \sqrt{-\boldsymbol{\Delta}}) u_{0}, \frac{\sin (t \sqrt{-\boldsymbol{\Delta}})}{\sqrt{-\boldsymbol{\Delta}}} u_{1}\right)+v=u_{l}+v
$$

$$
v \in C^{0}\left((0, T) ; H^{2}(M)\right) \cap C^{1}\left((0, T) ; H^{1}(M)\right) .
$$

On cherche alors $v$ vérifiant

$$
\left(\partial_{t}^{2}-\boldsymbol{\Delta}\right) v=-\left(u_{l}+v\right)^{p},\left.\quad v\right|_{t=0}=\left.\partial_{t} v\right|_{t=0}=0
$$

soit

$$
v(t)=-\int_{0}^{t} \frac{\sin ((t-s) \sqrt{-\boldsymbol{\Delta}})}{\sqrt{-\boldsymbol{\Delta}}}\left(u_{l}+v\right)^{p}(s) d s .
$$

Soit $r>4$ grand et $T \leq 1$. Notons $\left\|u_{l}\right\|_{W^{1, r}}=\left\|u_{l}\right\|_{W^{1, r}((0,1) \times M)}$. D'après la proposition C.3, $\left\|u_{l}\right\|_{W^{1, r}}$ est fini $\mathbb{P}$-presque sûrement et, d'après les injections de Sobolev $H^{2}(M) \rightarrow L^{\infty}(M)$ et $W^{1, r}((0,1) \times M) \rightarrow L^{\infty}((0,1) \times M)$ pour $r>4$, on a pour $s \in[0, T]$

$$
\begin{aligned}
\left\|\left(u_{l}+v\right)^{p}(s)\right\|_{H^{1}(M)} & \leq C\left(\left\|u_{l}(s)\right\|_{L^{\infty}}+\|v(s)\|_{L^{\infty}}\right)^{p-1}\left(\left\|u_{l}(s)\right\|_{H^{1}}+\|v(s)\|_{H^{1}}\right) \\
& \leq C\left(\left\|u_{l}\right\|_{W^{1, r}}+\|v(s)\|_{H^{2}}\right)^{p-1}\left(\left\|u_{l}(s)\right\|_{H^{1}}+\|v(s)\|_{H^{2}}\right)
\end{aligned}
$$

et de même, pour $\sup _{0 \leq s \leq T}\left\|v_{j}(s)\right\|_{H^{2}} \leq R$ et $s \in[0, T]$,

$$
\begin{aligned}
& \left\|\left(u_{l}+v_{1}\right)^{p}(s)-\left(u_{l}+v_{2}\right)^{p}(s)\right\|_{H^{1}(M)} \\
& \left.\quad \leq C\left(\left\|u_{l}\right\|_{W^{1, r}}+R\right)^{p-2}\right)\left(\left\|u_{l}\right\|_{W^{1, r}}+R+\left\|u_{l}(s)\right\|_{H^{1}}\right)\left(\left\|v_{1}(s)-v_{2}(s)\right\|_{H^{2}}\right) .
\end{aligned}
$$


Comme on a $\int_{0}^{T}\left\|u_{l}(s)\right\|_{H^{1}} d s \leq C \sqrt{T}\left\|u_{l}\right\|_{W^{1, r}}$, on déduit du théorème du point fixe qu'il existe $c>0$ tel que l'équation (5.5) admet un unique point fixe dans la boule de rayon $R$ de

$$
X_{T}=C^{0}\left((0, T) ; H^{2}(M)\right)
$$

dès que

$$
T R^{p-1}+\sqrt{T}\left\|u_{l}\right\|_{W^{1, r}}^{p-1}+\sqrt{T} R^{p-2}\left\|u_{l}\right\|_{W^{1, r}} \leq c, \text { et } \sqrt{T}\left\|u_{l}\right\|_{W^{1, r}}^{p} \leq c R .
$$

Comme il existe $\alpha>0, \beta>0$ petits tels que ces inéquations ont une solution en $R$ pour $\left\|u_{l}\right\|_{W^{1, r}} \leq \alpha T^{-\beta}$, on déduit (5.4) de (C.9) et (C.10). À nouveau d'après (C.9) et (C.10), $u_{l}$ vérifie (5.3). De plus, d'après (5.5), et puisque $\left(u_{l}+v\right) \in L^{1}\left((0, T), H^{1}(M)\right)$, les inégalités de Strichartz pour l'équation des ondes en dimension 3 impliquent, pour $\frac{1}{q}+\frac{3}{r}=\frac{1}{2}$ et $q \in] 2, \infty]$, qu'on a $v \in L^{q}\left((0, T), W^{1, r}(M)\right)$ et $\partial_{t} v \in L^{q}\left((0, T), L^{r}(M)\right)$, donc $v$ vérifie (5.3). Pour démontrer l'identité d'énergie, il suffit de considérer la suite de données initiales régularisées $\left(u_{0, n}, u_{1, n}\right)=\chi\left(2^{-n} \Delta\right)\left(u_{0}, u_{1}\right), \chi \in C_{0}^{\infty}(\mathbb{R})$ égale à 1 près de 0 . La famille d'opérateurs $\chi\left(2^{-n} \Delta\right)$ est uniformément bornée sur $W^{1, r}$. On en déduit que la démonstration que nous venons de donner garantit l'existence d'une solution (régulière) pour la famille de solutions associées aux données initiales $\left(u_{0, n}, u_{1, n}\right)$ sur un intervalle de temps uniforme. Évidemment ces solutions régulières vérifient l'identité d'énergie, et de plus on a

$$
\left(\partial_{t}^{2}-\Delta\right)\left(v_{n}-v\right)=\left(u_{l}+v\right)^{p}-\left(u_{l, n}-v_{n}\right)^{p}
$$

dont on déduit

$$
\begin{aligned}
& \left\|\left(u_{l}+v\right)^{p}-\left(u_{l, n}-v_{n}\right)^{p}\right\|_{H^{1}(M)} \\
& \leq C\left(\left\|u_{l}-u_{l, n}\right\|_{W^{1, p}}+\left\|v-v_{n}\right\|_{H^{2}}\right)\left(\left\|u_{l}\right\|_{W^{1, p}}+\left\|u_{l, n}\right\|_{W^{1, p}}+\|v\|_{H^{2}}+\left\|v_{n}\right\|_{H^{2}}\right),
\end{aligned}
$$

ce qui implique, puisque

$$
\lim _{n \rightarrow+\infty}\left\|u_{l}-v_{n, l}\right\|_{W^{1, p}((0, T) \times M)}=0,
$$

quitte à réduire l'intervalle de temps, que

$$
\lim _{n \rightarrow+\infty}\left\|v-v_{n}\right\|_{L^{\infty}\left((0, T) ; H^{2}\right)}=0 .
$$

On en déduit que $u$ vérifie aussi l'identité d'énergie. La preuve du théorème 14 est complète.

Théorème 15 (Unicité fort-faible). - Pour $\mathbb{P}$-presque toutes données initiales $\left(u_{0}, u_{1}\right)$ dans l'espace d'énergie, $H^{1}(M) \times L^{2}(M)$, on a $u_{0} \in L^{p+1}$ et toute solution faible de (5.1) vérifiant l'inégalité d'énergie (5.2) coïncide avec la solution donnée par le théorème 14 sur l'intervalle de temps $(0, T)$.

Démonstration. - Le théorème 15 est une conséquence directe du résultat de stabilité suivant :

Proposition 5.1. - Soit u une solution forte de l'équation des ondes (5.1) définie sur $[0, T]$, de données initiales $\left(u_{0}, u_{1}\right)$ vérifiant les estimées (5.3) et limite de solutions régulières pour la norme donnée par (5.3). Il existe $C>0$ tel que pour toute solution (faible) $v$ sur 
l'intervalle $[0, T]$ de l'équation (5.1), de données initiales $\left(v_{0}, v_{1}\right) \in\left(H^{1}(M) \cap L^{p+1}(M)\right) \times$ $L^{2}(M)$ vérifiant

$$
\forall t \in[0, T] \quad \mathcal{E}(v)(t) \leq \mathcal{E}(v)(0)
$$

on a

$$
\mathcal{E}(u-v)(t)+\|u-v\|_{L^{2}(M)}^{2}(t) \leq C\left(\mathcal{E}(u-v)(0)+\|u-v\|_{L^{2}(M)}^{2}(0)\right) .
$$

Pour démontrer cette proposition, on reprend et on affine un résultat similaire de Struwe [34] (démontré pour des solutions fortes $C^{\infty}$ ), (il faut modifier la preuve pour l'adapter au niveau de régularité des solutions fortes données par le théorème 14). On notera $w=v-u$ et $D w=\left(\partial_{t} w, \nabla_{x} w\right)$. On a

$$
\left(\partial_{t}^{2} w-\boldsymbol{\Delta}_{x} w\right)+(u+w)^{p}-u^{p}=0
$$

et

$$
\mathcal{E}(v)=\mathcal{E}(u)+I+I I
$$

avec

$$
\begin{aligned}
I & =\int_{M} D u \cdot D w+u^{p} w \\
I I & =\int_{M} \frac{|D w|^{2}}{2}+\frac{(u+w)^{p+1}-u^{p+1}}{p+1}-u^{p} w .
\end{aligned}
$$

Un calcul (justifié par une régularisation de $u$ et un passage à la limite) donne

$$
\frac{d}{d t} I(t)=\int_{M}\left(u^{p}+p u^{p-1} w-(u+w)^{p}\right) \partial_{t} u
$$

On va montrer qu'il existe une fonction $g(t) \in L^{2} \subset L^{1}$ telle qu'on ait

$$
\left|\frac{d}{d t} I(t)\right| \leq C\left(\mathcal{E}(w)+\|w\|_{L^{2}}^{2}\right)(t) g(t) .
$$

Supposons provisoirement (5.11) démontré. Un calcul simple montre que

$$
\frac{(u+w)^{p+1}-u^{p+1}}{p+1}-u^{p} w \geq \frac{1}{C} w^{p+1}-C w^{2}
$$

ce qui implique

$$
I I(t) \geq \frac{1}{C} \mathcal{E}(w)(t)-C\|w\|_{L^{2}}^{2}(t)
$$

Si on revient à l'hypothèse de décroissance de l'énergie de $v$, on obtient

$$
0 \leq \mathcal{E}(v)(0)-\mathcal{E}(v)(t) \Rightarrow I I(t) \leq I I(0)+I(0)-I(t) .
$$

Comme pour $q$ assez grand, $u_{0} \in W^{1, q}(M) \subset L^{\infty}(M)$, on a

$$
I I(0)=\mathcal{E}(w)(0)+\frac{1}{p+1} \sum_{q=2}^{p+1}\left(\begin{array}{l}
p \\
q
\end{array}\right) w^{q} u^{p+1-q} ;
$$

en utilisant que $u$ est bornée dans $L^{\infty}((0, T) \times M)$ et

$$
\int_{M}|w|^{l} \leq \int_{M}|w|^{p+1} 1_{|w| \geq 1}+|w|^{2} 1_{|w|<1} \leq \mathcal{E}(w)+\|w\|_{L^{2}}^{2}
$$

on obtient

$$
|I I(0)| \leq C\left(\mathcal{E}(w)(0)+\|w\|_{L^{2}}^{2}(0)\right)
$$


ceci implique d'après (5.11), (5.12), et (5.13),

$$
\mathcal{E}(w)(t) \leq C\left(\int_{0}^{t}\left(\mathcal{E}(w)+\|w\|_{L^{2}}^{2}\right)(s) g(s) d s+\|w\|_{L^{2}}^{2}(t)+\mathcal{E}(w)(0)+\|w\|_{L^{2}}^{2}(0)\right)
$$

mais d'après l'inégalité de Minkowski,

$$
\|w\|_{L^{2}}^{2}(t) \leq C\left(\int_{0}^{t}\left\|\partial_{t} w\right\|_{L^{2}}^{2}(s) d s+\|w\|_{L^{2}}^{2}(0)\right)
$$

et donc

$$
\left(\mathcal{E}(w)+\|w\|_{L^{2}}^{2}\right)(t) \leq C\left(\int_{0}^{t}\left(\mathcal{E}(w)+\|w\|_{L^{2}}^{2}\right)(s)(1+g(s)) d s+\mathcal{E}(w)(0)+\|w\|_{L^{2}}^{2}(0)\right) .
$$

Le lemme de Gronwall démontre alors la proposition 5.1.

Montrons à présent (5.11). On note $J(t)=\left(\mathcal{E}(w)+\|w\|_{L^{2}}^{2}\right)(t)$. Le terme $\frac{d}{d t} I(t)$ est combinaison linéaire de termes $\int_{M} w^{l} u^{p-l} \partial_{t} u$ avec $l \in\{2, \ldots, p\}$. D'après (5.3), on a $u^{p-l} \partial_{t} u \in L^{2}\left((0, T) ; L^{r}(M)\right)$ pour tout $r<\infty$. Il suffit donc de vérifier qu'on a pour tout $l \in\{2, \ldots, p\}$, et pour $f \in \cap_{r<\infty} L^{2}\left((0, T) ; L^{r}(M)\right)$,

$$
\left|\int_{M} w^{l} f\right| \leq J(t) g(t), \text { avec } g \in L^{2} .
$$

Choisissons pour $j \in \mathbb{N}$ des $\varphi_{j}$ tels que $\sum_{j} \varphi_{j}^{2}\left(2^{-j} s\right)=1, \varphi_{0}$ à support dans [0,3], $\varphi_{j}$ à support dans $[1,5]$, la famille des $\varphi_{j}$ étant uniformément $C^{\infty}$. Soit $A_{j}=\varphi_{j}\left(2^{-j} \sqrt{-\triangle}\right)$. Alors les opd autoadjoints $A_{j}$ sont uniformément en $j$ bornés sur tous les $L^{q}, q \in[1, \infty]$, et on a $\sum A_{j}^{2}=\mathrm{Id}$. Il en résulte $\int_{M} w^{l} f=\sum_{j} \int_{M} A_{j}\left(w^{l}\right) A_{j}(f)$. Par hypothèse sur la fonction $f$, on a pour tout $r<\infty$

$$
\left\|A_{j}(f)\right\|_{L^{\infty}(M)} \leq 2^{j d / r} g_{r}(t), \text { avec } g_{r} \in L^{2} .
$$

Soit $\chi(s) \in C^{\infty}$ égal à 1 pour $s \leq 1$ et nul pour $s \geq 2$. On a, avec $\lambda_{j}>0$

$$
A_{j}\left(w^{l}\right)=A_{j}\left(w^{l} \chi\left(\frac{w^{2}}{\lambda_{j}^{2}}\right)\right)+A_{j}\left(w^{l}(1-\chi)\left(\frac{w^{2}}{\lambda_{j}^{2}}\right)\right)=I_{j}+I I_{j} .
$$

On a, avec $C$ indépendant de $j$

$$
\left\|I I_{j}\right\|_{L^{1}(M)} \leq C\left\|w^{l}(1-\chi)\left(\frac{w^{2}}{\lambda_{j}^{2}}\right)\right\|_{L^{1}(M)} \leq \frac{C}{\lambda_{j}^{p+1-l}}\|w\|_{L^{p+1}(M)}^{p+1} \leq \frac{C J(t)}{\lambda_{j}^{p+1-l}}
$$

et aussi, en utilisant $l \geq 2$ et $\left\|w \nabla_{x} w\right\|_{L^{1}} \leq\|w\|_{L^{2}}\left\|\nabla_{x} w\right\|_{L^{2}}$

$$
\begin{aligned}
\left\|\nabla_{x} I_{j}\right\|_{L^{1}(M)} & \leq \| A_{j}\left(\nabla_{x}\left(w^{l} \chi\right)\left\|_{L^{1}(M)}+\right\|\left[A_{j}, \nabla_{x}\right]\left(w^{l} \chi\right) \|_{L^{1}(M)}\right. \\
& \leq C \lambda_{j}^{l-2}\left(\|w\|_{L^{2}}\left\|\nabla_{x} w\right\|_{L^{2}}+\|w\|_{L^{2}}^{2}\right) \leq C \lambda_{j}^{l-2} J(t) .
\end{aligned}
$$

En écrivant

$$
\int_{M} w^{l} f=\int_{M} A_{0}\left(w^{l}\right) A_{0}(f)+\sum_{j \geq 1} \int_{M} I I_{j}\left(w^{l}\right) A_{j}(f)+\sum_{j \geq 1} \int_{M} \triangle I_{j}\left(w^{l}\right) \triangle^{-1} A_{j}(f)
$$

on obtient donc en utilisant (5.16), (5.18) et (5.19), et $\left|\int_{M} A_{0}\left(w^{l}\right) A_{0}(f)\right| \leq C J(t) g_{r}(t)$

$$
\left|\int_{M} w^{l} f\right| \leq C J(t) g_{r}(t)\left(1+\sum_{j \geq 1} \frac{2^{j d / r}}{\lambda_{j}^{p+1-l}}+\sum_{j \geq 1} 2^{-j} 2^{j d / r} \lambda_{j}^{l-2}\right),
$$

ce qui prouve (5.15) avec le choix $\left.\lambda_{j}=2^{j a}, a \in\right] 0, \frac{1}{p-2}[$ et $r$ grand. 


\section{Cas des variétés à bord}

Dans le cas où la variété $M$ à un bord tel que $M \cup \partial M$ est compacte, une bonne partie des résultats exposés dans cet article reste vraie si on considère le laplacien sur $(M, g)$ avec conditions de Dirichlet ou Neumann. En effet, la formule de Weyl avec reste précisé (1.2) reste vraie d'après les travaux d'Ivrii [21], donc les minorations/majorations de (2.7) aussi. En ce qui concerne l'estimée $e_{x, h} \leq C_{0} N_{h}$, elle est conséquence, dans le cas avec bord, des travaux de Sogge [33] pour les conditions aux limites de Dirichlet, puis Smith-Sogge [31] pour les conditions de Neuman. En effet, d'après [33, Proposition 2.3], on obtient

Proposition 6.1. - Supposons que $a_{h}=1, b_{h}=1+h$. Alors il existe $C>0$ tel que, pour tout $x \in M$ et tout $0<h \leq 1$,

$$
\left\|\Pi_{h}\right\|_{L^{1}(M) \rightarrow L^{\infty}(M)} \leq C h^{1-d} .
$$

Il suffit ensuite dans le cas général de recouvrir l'intervalle $\left(a_{h}, b_{h}\right)$ par un nombre fini (d'ordre $\left(b_{h}-a_{h}\right) / h$ ) d'intervalles de taille $h$, d'appliquer la proposition 6.1 à chacun de ces intervalles pour obtenir dans le cas général

$$
\left\|\Pi_{h}\right\|_{L^{1}(M) \rightarrow L^{\infty}(M)} \leq C h^{-d}\left(b_{h}-a_{h}\right) .
$$

Puis, le noyau de l'opérateur $\Pi_{h}$ étant donné par

$$
K_{h}(x, y)=\sum_{k \in I_{h}} e_{k}(x) \overline{e_{k}(y)}
$$

on obtient

$$
\sup _{x \in M}\left|e_{x, h}\right| \leq\left\|K_{h}\right\|_{L^{\infty}(M \times M)}=\left\|\Pi_{h}\right\|_{L^{1}(M) \rightarrow L^{\infty}} \leq C h^{-d}\left(b_{h}-a_{h}\right) .
$$

On en déduit que les résultats des sections 2.1, 2.3 ainsi que la remarque 3.3 restent valides dans ce cadre. Il est possible que les résultats de la section 5 puissent aussi être étendus à ce cadre, mais la plage des estimations de Strichartz valides dans ce cadre des problèmes aux limites étant plus restreinte (voir [11, 20, 6]), cela nécessiterait une analyse plus précise.

\section{Appendice A}

\section{Calcul des probabilités sur les sphères}

On note $L(d x)$ la mesure de Lebesgue sur $\mathbb{R}^{N}$ et $p_{N}$ la probabilité uniforme sur la sphère unité $S(N)$ de $\mathbb{R}^{N}$. La probabilité $p_{N}$ est la mesure image de $\Pi_{1 \leq j \leq N} \frac{1}{\sqrt{2 \pi}} e^{-\left|x_{j}\right|^{2} / 2} L\left(d x_{j}\right)$ par l'application $\pi$

$$
x=\left(x_{1}, \ldots, x_{N}\right) \mapsto \pi(x)=a=\left(a_{1}, \ldots, a_{N}\right) \in S(N), \quad a_{j}=\frac{x_{j}}{\sqrt{\sum x_{l}^{2}}} .
$$

En effet, $\pi_{*}\left(\Pi_{1 \leq j \leq N} \frac{1}{\sqrt{2 \pi}} e^{-x_{j}^{2} / 2} L\left(d x_{j}\right)\right)$ est une probabilité sur $S(N)$ invariante par les isométries de $S(N)$ puisque, pour tout $g \in S O(N), g \pi=\pi g$, et $\Pi_{1 \leq j \leq N} \frac{1}{\sqrt{2 \pi}} e^{-x_{j}^{2} / 2} L\left(d x_{j}\right)=$ $(2 \pi)^{-N / 2} e^{-|x|^{2} / 2} L(d x)$ est invariante par l'action de $S O(N)$. 
Soit $M \geq 1$ fixé. Pour $N_{j} \geq 1$, soit $N=N_{1}+\cdots+N_{M}$. La mesure de Lebesgue sur $\mathbb{R}^{N}=\Pi_{j} \mathbb{R}^{N_{j}}$ est donnée dans les coordonnées $x=\left(x_{1}, \ldots, x_{M}\right), x_{j}=\rho_{j} \omega_{j} \in \mathbb{R}^{N_{j}}$, avec $\rho_{j}>0, \omega_{j} \in S\left(N_{j}\right)$ par la formule

$$
\Pi_{j=1}^{M} \rho_{j}^{N_{j}-1} d \rho_{j} \otimes c_{N_{j}} p_{N_{j}}\left(\omega_{j}\right)
$$

où $c_{N}$ est le volume de $S(N)$. En particulier, l'application $|\pi|_{M}$ de $S(N)$ dans la sphère réelle $S(M-1) \subset \mathbb{R}^{M}$

$$
x \mapsto|\pi|_{M}(x)=\left(\left|x_{1}\right|, \ldots,\left|x_{M}\right|\right)
$$

envoie $p_{N}$ sur la probabilité

$$
\left(|\pi|_{M}\right)_{*} p_{N}=\frac{\Pi_{j} c_{N_{j}}}{c_{N}} \Pi_{j} 1_{\rho_{j} \geq 0} \rho_{j}^{N_{j}-1} d \sigma(\rho)
$$

où $d \sigma(\rho)$ est la mesure de Lebesgue sur $S(M)$.

En choisissant $M=2, N_{1}=2, N_{2}=N-2$, on a donc pour $x=\left(x_{1}, x_{2}\right) \in S(N)$, et pour $t=\cos \theta_{0} \in[0,1], \theta_{0} \in[0, \pi / 2]$,

$$
p_{N}\left(\left|x_{1}\right|>t\right)=\frac{c_{2} c_{N-2}}{c_{N}} \int_{0}^{\theta_{0}} \cos \theta(\sin \theta)^{N-3} d \theta=\frac{c_{2} c_{N-2}}{c_{N}(N-2)}\left(\sin \theta_{0}\right)^{N-2} .
$$

En choisissant $\theta_{0}=\pi / 2$, on obtient

$$
p_{N}\left(\left|x_{1}\right|>0\right)=\frac{c_{2} c_{N-2}}{c_{N}(N-2)}=1
$$

d'où

$$
p_{N}\left(\left|x_{1}\right|>t\right)=\mathbf{1}_{t \in[0,1[}\left(1-t^{2}\right)^{\frac{N}{2}-1} .
$$

Rappelons aussi le résultat suivant de concentration de la mesure (voir par exemple [25, Theorem 2.3 et (1.10), (1.12)]).

Proposition A.1. - Considérons une fonction F Lipschitz sur la sphère $\mathbb{S}^{d}=S(d+1)$ (munie de sa distance géodésique naturelle et de la mesure de probabilité uniforme, $\mu$ ). On définit sa médiane $\mathcal{M}(F)$ par la relation

$$
\mu(F \geq \mathcal{M}(F)) \geq \frac{1}{2}, \quad \mu(F \leq \mathcal{M}(F)) \geq \frac{1}{2} .
$$

Alors, pour tout $r>0$,

$$
\mu(|F-\mathcal{M}(F)|>r) \leq 2 e^{-(d-1) \frac{r^{2}}{2\|F\|_{\text {Lips }}^{2}}} .
$$

\section{Appendice B}

\section{Calcul $h$-pseudodifférentiel}

Nous rappelons ici les bases du calcul $h$-pseudo-différentiel sur $M$, pour lesquelles nous renvoyons à [27]. Pour $m \in \mathbb{R}$, soit $S^{m}$ l'espace des fonctions $a(x, \xi, h)$ de classe $C^{\infty}$ en $(x, \xi) \in \mathbb{R}^{2 d}$, dépendantes du paramètre $\left.\left.h \in\right] 0,1\right]$ telles que, pour tout $\alpha, \beta$, il existe $C_{\alpha, \beta}$ tel que pour tout $(x, \xi) \in \mathbb{R}^{2 d}$ et tout $\left.\left.h \in\right] 0,1\right]$ on a

$$
\left|\partial_{x}^{\alpha} \partial_{\xi}^{\beta} a(x, \xi, h)\right| \leq C_{\alpha, \beta}(1+|\xi|)^{m-|\beta|} .
$$

$4^{\text {e }}$ SÉRIE - TOME $46-2013-$ N $^{\circ} 6$ 
Pour $a \in S^{m}$, on note $\operatorname{Op}(a)$ l'opérateur h-pseudodifférentiel agissant sur l'espace de Schwartz $\phi\left(\mathbb{R}^{d}\right)$ par

$$
\mathrm{Op}(a)(f)(x)=(2 \pi h)^{-d} \int e^{i(x-y) \xi / h} a(x, \xi, h) f(y) d y d \xi .
$$

Rappelons que pour $a \in S^{0}$, l'opérateur $\operatorname{Op}(a)$ est uniformément en $h$ borné sur $L^{2}\left(\mathbb{R}^{d}\right)$ et que pour $a \in S^{m}, b \in S^{k}$, on a $\operatorname{Op}(a) \mathrm{Op}(b)=\mathrm{Op}(c)$ où $c=a \sharp b \in S^{m+k}$ est donné par l'intégrale oscillante

$$
c(x, \xi, h)=(2 \pi h)^{-d} \int e^{-i z \theta / h} a(x, \xi+\theta, h) b(x+z, \xi, h) d z d \theta
$$

et admet le développement asymptotique

$$
c(x, \xi, h)=\sum_{|\alpha|<N} \frac{h^{|\alpha|}}{i^{|\alpha|} \alpha !} \partial_{\xi}^{\alpha} a(x, \xi, h) \partial_{x}^{\alpha} b(x, \xi, h)+h^{N} r_{N}(x, \xi, h), \quad r_{N} \in S^{m+l-N}
$$

Le sous-espace $S_{\mathrm{cl}}^{m}$ de $S^{m}$ est l'ensemble des $a(x, \xi, h) \in S^{m}$ tels qu'il existe une suite $a_{n}(x, \xi) \in S^{m-n}, n \geq 0$ telle que, pour tout $N$, on a

$$
a(x, \xi, h)=\sum_{0 \leq n<N}(h / i)^{n} a_{n}(x, \xi)+h^{N} r_{N}(x, \xi, h), \quad r_{n} \in S^{m-N} .
$$

D'après (B.4), on a $a \sharp b \in S_{\mathrm{cl}}^{m+k}$ pour $a \in S_{\mathrm{cl}}^{m}$ et $b \in S_{\mathrm{cl}}^{k}$.

Soit $e_{j}(x) \in C^{\infty}(M), j \geq 0$ une base orthonormale dans $L^{2}\left(M, d_{g} x\right)$ de fonctions propres de $-\boldsymbol{\Delta}$ avec $-\Delta e_{j}=\omega_{j}^{2} e_{j}$. Pour toute distribution $f \in \mathscr{D}^{\prime}(M)$, on pose $c_{j}(f)=\int f e_{j} d_{g} x$ de sorte qu'on a $f(x)=\sum_{j} c_{j}(f) e_{j}(x)$, la série étant convergente dans $\mathscr{D}^{\prime}(M)$. Pour $s \in \mathbb{R}$, soit $H^{s}(M)=\left(1-\boldsymbol{\Delta}_{g}\right)^{-s / 2} L^{2}\left(M, d_{g} x\right)$ l'espace de Sobolev usuel sur $M$. Pour $f \in \mathscr{D}^{\prime}(M)$ on a $f \in H^{s}(M)$ ssi $\|f\|_{H^{s}(M)}^{2}=\sum_{j}\left(1+\omega_{j}^{2}\right)^{s}\left|c_{j}(f)\right|^{2}<\infty$. On utilise aussi les normes semi-classiques $H^{s}$ définies par

$$
\|f\|_{h, s}^{2}=\sum_{j}\left(1+h^{2} \omega_{j}^{2}\right)^{s}\left|f_{j}\right|^{2} .
$$

Une famille d'opérateurs $\left.\left.R_{h}, h \in\right] 0,1\right]$, opérant sur $\mathscr{D}^{\prime}(M)$ est dite régularisante ssi pour tout $s, t, N, R_{h}$ envoie $H^{s}(M)$ dans $H^{t}(M)$ et il existe $C_{s, t, N}$ tel que pour tout $\left.\left.h \in\right] 0,1\right]$ on a

$$
\left\|R_{h}(f)\right\|_{H^{t}(M)} \leq C_{s, t, N} h^{N}\|f\|_{H^{s}(M)} .
$$

Une famille d'opérateurs $\left.\left.A_{h}, h \in\right] 0,1\right]$ agissant sur $\mathscr{D}^{\prime}(M)$ appartient à l'espace $\varepsilon_{\mathrm{cl}}^{m}$ des opérateurs $h$-pseudodifférentiels d'ordre $m$, ssi pour tout $x_{0} \in M$, il existe un ouvert de carte $U$ centré en $x_{0}$ et deux fonctions $\varphi, \psi \in C_{0}^{\infty}(U)$ égales à 1 près de $x_{0}$ avec $\psi$ égale à 1 près du support de $\varphi$ telles que $A_{h} \varphi=\psi A_{h} \varphi+R_{h}$, avec $R_{h}$ régularisant et il existe $a \simeq \sum_{n \geq 0}(h / i)^{n} a_{n}(x, \xi) \in S_{\mathrm{cl}}^{m}$ tel que, dans l'ouvert de carte $U$, on a $\psi A_{h} \varphi=\operatorname{Op}(a)$. Le symbole principal de $A_{h}, \sigma_{0}\left(A_{h}\right)(x, \xi)$, est par définition le premier terme $a_{0}(x, \xi) \mathrm{du}$ développement asymptotique de $a(x, \xi, h)$. C'est une fonction intrinsèque sur $T^{*} M$, et pour toute fonction lisse $\varphi \in C^{\infty}(M)$, on a

$$
e^{-i \varphi(x) / h} A_{h}\left(e^{i \varphi(x) / h}\right)=\sigma_{0}\left(A_{h}\right)(x, d \varphi(x))+\theta(h) .
$$

Le support essentiel de $A_{h}$ est dit contenu dans le compact $K$ de $T^{*} M$ si on a avec les notations précédentes et dans tout ouvert de carte $U, a \in S^{-\infty}$, et $a_{n}(x, \xi)$ est à support dans $K$ pour tout $n$. 
$\mathcal{E}_{\mathrm{cl}}=\cup_{m} \delta_{\mathrm{cl}}^{m}$ est l'algèbre des opérateurs $h$-pseudodifférentiels classiques sur $M$. Pour $A_{h} \in \mathcal{E}_{\mathrm{cl}}^{m}$ et $B_{h} \in \mathcal{E}_{\mathrm{cl}}^{k}$, on a $A_{h} B_{h} \in \mathcal{E}_{\mathrm{cl}}^{m+k}, \sigma_{0}\left(A_{h} B_{h}\right)=\sigma_{0}\left(A_{h}\right) \sigma_{0}\left(B_{h}\right)$ et le commutateur $\left[A_{h}, B_{h}\right]=A_{h} B_{h}-B_{h} A_{h}$ vérifie $\left[A_{h}, B_{h}\right] \in h \mathcal{E}_{\mathrm{cl}}^{m+k-1}, \sigma_{0}\left(\frac{i}{h}\left[A_{h}, B_{h}\right]\right)=$ $\left\{\sigma_{0}\left(A_{h}\right), \sigma_{0}\left(B_{h}\right)\right\}$ où $\{f, g\}$ est le crochet de Poisson. De plus, pour tout $A_{h} \in \mathcal{E}_{\mathrm{cl}}^{m}$, on a $A_{h}^{*} \in \mathcal{E}_{\mathrm{cl}}^{m}, \sigma_{0}\left(A_{h}^{*}\right)=\overline{\sigma_{0}\left(A_{h}\right)}$, et pour tout $s \in \mathbb{R}$, il existe $C_{s}$ indépendant de $\left.\left.h \in\right] 0,1\right]$ tel que

$$
\left\|A_{h} f\right\|_{h, s-m} \leq C_{s}\|f\|_{h, s} \quad \forall f \in H^{s}(M) .
$$

De plus, si $A_{h} \in \mathcal{E}_{\mathrm{cl}}^{-\infty}=\cap_{m} \mathcal{E}_{\mathrm{cl}}^{m}$, il existe $C$ indépendant de $\left.\left.h \in\right] 0,1\right]$ et $p \in[1, \infty]$ tel que

$$
\left\|A_{h} f\right\|_{L^{p}(M)} \leq C\|f\|_{L^{p}(M)} \quad \forall f \in L^{p}(M) .
$$

Rappelons enfin que, pour tout $\phi \in C_{0}^{\infty}\left(\left[0, \infty[)\right.\right.$, l'opérateur $\phi\left(-h^{2} \boldsymbol{\Delta}\right)$ défini par

$$
\phi\left(-h^{2} \boldsymbol{\Delta}\right)(f)=\sum_{j} \phi\left(h^{2} \omega_{j}^{2}\right) c_{j}(f) e_{j}(x)
$$

appartient à $\mathcal{E}_{\mathrm{cl}}^{-\infty}$, et que son symbole principal est

$$
\sigma_{0}\left(\phi\left(-h^{2} \boldsymbol{\Delta}_{g}\right)\right)=\phi\left(|\xi|_{x}^{2}\right)
$$

où $|\xi|_{x}$ est la longueur riemannienne du covecteur $\xi$ en $x$. Pour une preuve de ce fait, on renvoie à [14].

\section{Appendice C}

\section{Quelques propriétés des mesures}

Dans cet appendice, nous étudions quelques propriétés des mesures que nous avons définies. Nous renvoyons à $[7,28]$ pour des résultats connexes.

\section{C.1. Mesures sur l'espace d'énergie}

Soit $0<a_{0}<a_{1}<\cdots$ une suite strictement croissante telle que $\lim _{k \rightarrow \infty} a_{k}=\infty$, et telle qu'il existe $C>1$ tel que

$$
\forall k \geq 0, \quad a_{k+1} \leq C a_{k} .
$$

On pose $a_{-1}=-1$ et pour tout $k \geq 0$

$$
E_{k}=\left\{u \in L^{2}(M) ; u(x)=\sum_{j \in I_{k}} z_{j} e_{j}(x)\right\}, \quad I_{k}=\left\{j ; a_{k-1}<\omega_{j} \leq a_{k}\right\} .
$$

On a alors $L^{2}(M)=\oplus_{k \geq 0} E_{k}$ et pour $u=\sum_{k \geq 0} u_{k}, u_{k} \in E_{k}$ et tout $s \geq 0$, quitte à augmenter $C$ pour avoir $1+a_{0}^{2} \leq C^{2}$

$$
C^{-2 s} \sum_{k \geq 0}\left(1+a_{k}^{2}\right)^{s}\left\|u_{k}\right\|_{L^{2}}^{2} \leq\|u\|_{H^{s}}^{2} \leq \sum_{k \geq 0}\left(1+a_{k}^{2}\right)^{s}\left\|u_{k}\right\|_{L^{2}}^{2} .
$$

Ces inégalités sont renversées pour $s \leq 0$. 
On se donne une suite $\left(\alpha_{k}\right)_{k \geq 0}$ de réels positifs. On se donne également pour tout $k \in \mathbb{N}$ une mesure de probabilité sur $\mathbb{R}^{+}, p_{k}(r)$. On supposera qu'il existe $\gamma>0$ tel que

$$
\left(H_{\gamma}\right) \quad \exists C, c>0 ; \forall k \in \mathbb{N}, \int_{\rho}^{+\infty} d p_{k}(r) \leq C e^{-c \rho^{\gamma}} .
$$

Par convention, on supposera que $H_{\infty}$ est vérifiée si les mesures $d p_{k}$ sont supportées dans un compact fixe (indépendant de $k$ ). Par exemple $d p_{k}^{i}=\delta_{r=r_{0}}$ vérifie $H_{\infty}$ et $d p_{k}^{i}=\sqrt{\frac{2}{\pi}} e^{-r^{2} / 2} d r$ vérifie $H_{2}$. On note $q_{k}$ l'image de $p_{k}$ par l'application $r \mapsto \alpha_{k} r$. On munit $E_{k}$ de la norme $L^{2}(M)$, on note $S_{k}$ sa sphère unité, et $P_{k}$ la probabilité uniforme sur $S_{k}$. On note $\nu_{k}$ la probabilité sur $E_{k}$ image de $q_{k} \otimes P_{k}$ par l'application $(r, \omega) \mapsto r \omega$ de $\mathbb{R}^{+} \times S_{k}$ dans $E_{k}$.

On notera $\mathbb{P}$ la mesure de probabilité définie sur l'espace $\Pi_{k \geq 0} E_{k}$ par

$$
\mathbb{P}=\otimes_{k} \nu_{k}
$$

et

$$
\left\|\left(\alpha_{k}\right)\right\|_{s}^{2}=\sum_{k} \alpha_{k}^{2}\left(1+a_{k}^{2}\right)^{s} \in[0,+\infty]
$$

On identifiera la suite $U=\left(u_{k}\right) \in \Pi_{k \geq 0} E_{k}$ avec la somme de la série $u=\sum_{k} u_{k}$ (on sera par la suite toujours dans un cadre où cette série converge dans $\mathscr{D}^{\prime}(M)$ ). On notera $\mathcal{M}_{s}$ l'ensemble des mesures de probabilité ainsi définies quand $\left(\alpha_{k}\right)$ décrit l'ensemble des suites vérifiant $\left\|\left(\alpha_{k}\right)\right\|_{s}<+\infty$. On a alors la

Proposition C.1. - Supposons que

$$
\left\|\left(\alpha_{k}\right)\right\|_{s}<+\infty
$$

Alors l'espace $H^{s}(M)$ est de mesure pleine $: \mathbb{P}\left(H^{s}(M)\right)=1$. Réciproquement, supposons que

$$
\left\|\left(\alpha_{k}\right)\right\|_{s}=+\infty
$$

et que les mesures $p_{k}$ ne se concentrent pas en 0 :

$$
\exists \rho>0, \delta<1 ; \forall k \in \mathbb{N}, p_{k}([0, \rho)) \leq \delta
$$

(on remarquera que cette dernière condition est toujours vérifiée si les mesures $p_{k}$ sont identiquement distribuées et non égales à $\left.\delta_{r=0}\right)$. Alors

$$
\mathbb{P}\left(\left\{U=\left(u_{k}\right) ; \sum_{k}\left\|u_{k}\right\|_{H^{s}(M)}^{2}<+\infty\right\}\right)=0 .
$$

Démonstration. - On calcule d'abord

$$
\begin{aligned}
\mathbb{E}\left(\|U\|_{H^{s}(M)}^{2}\right) & =\mathbb{E}\left(\sum_{k}\left\|u_{k}\right\|_{H^{s}(M)}^{2}\right)=\sum_{k} \mathbb{E}_{k}\left(\left\|u_{k}\right\|_{H^{s}(M)}^{2}\right) \\
& \leq C_{s} \sum_{k}\left(1+a_{k}^{2}\right)^{s} \int_{r=0}^{+\infty} r^{2} d q_{k} \leq C_{s} \sum_{k}\left(1+a_{k}^{2}\right)^{s} \alpha_{k}^{2}<+\infty,
\end{aligned}
$$


ce qui démontre que $\|u\|_{H^{s}}$ est finie presque sûrement. Réciproquement, sous les hypothèses (C.4) et (C.5), on a

$$
\begin{aligned}
\mathbb{E}\left(e^{-t\|U\|_{H^{s}}^{2}}\right) & =\prod_{k=1}^{+\infty} \mathbb{E}_{k}\left(e^{-t\left\|u_{k}\right\|_{H^{s}}^{2}}\right) \\
& \leq \prod_{k=1}^{+\infty} \int_{0}^{+\infty} e^{-c_{s} t r^{2}\left(1+a_{k}^{2}\right)^{s}} d q_{k} \\
& =\prod_{k=1}^{+\infty} \int_{0}^{+\infty} e^{-c_{s} t r^{2} \alpha_{k}^{2}\left(1+a_{k}^{2}\right)^{s}} d p_{k} \\
& \leq \prod_{k=1}^{+\infty}\left(p _ { k } \left([0, \rho)+e^{-c_{s} t \rho^{2} \alpha_{k}^{2}\left(1+a_{k}^{2}\right)^{s}}\left(1-p_{k}([0, \rho))\right)\right.\right. \\
& \leq \prod_{k=1}^{+\infty}\left[1-\left(1-p_{k}([0, \rho))\left(1-e^{-c_{s} t \rho^{2} \alpha_{k}^{2}\left(1+a_{k}^{2}\right)^{s}}\right)\right]\right.
\end{aligned}
$$

On remarque maintenant qu'on peut supposer

$$
\alpha_{k}^{2}\left(1+a_{k}^{2}\right)^{s} \rightarrow_{k \rightarrow+\infty} 0
$$

(car sinon le produit infini est clairement nul et le résultat est évident), et comme

$$
1-e^{-c_{s} t \rho^{2} \alpha_{k}^{2}\left(1+a_{k}^{2}\right)^{s}} \sim c_{s} t \rho^{2} \alpha_{k}^{2}\left(1+a_{k}^{2}\right)^{s}
$$

on a

$$
\left[1-\left(1-p_{k}([0, \rho))\left(1-e^{-c_{s} t \rho^{2} \alpha_{k}^{2}\left(1+a_{k}^{2}\right)^{s}}\right)\right] \sim\left[1-\left(1-p_{k}([0, \rho)) c_{s} t \rho^{2} \alpha_{k}^{2}\left(1+a_{k}^{2}\right)^{s}\right)\right]\right.
$$

et donc en prenant le logarithme dans (C.7), on obtient, d'après (C.5), que le produit infini est divergent vers 0 . On obtient donc

$$
\mathbb{E}\left(e^{-t\|U\|_{H^{s}}^{2}}\right)=0,
$$

et donc, $\mathbb{P}$-presque sûrement, $\|U\|_{H^{s}}^{2}=+\infty$.

\section{C.2. Critère d'orthogonalité}

Le critère suivant est dû à Kakutani [22].

ThÉORÈme (Kakutani). - On considère deux mesures $\mu_{1}, \mu_{2}$ associées au même choix de la suite $\left(a_{k}\right)$, mais à des suites $\left(\alpha_{k, 1}\right),\left(\alpha_{k, 2}\right)$ et $\left(d p_{k, 1}\right),\left(d p_{k, 2}\right)$ a priori différentes. On rappelle que les mesures $d q_{k, j}$ sont les images des mesures $d p_{k, j}$ par l'application $r \mapsto \alpha_{k, j} r$. Alors les mesures $\mu_{1}$ et $\mu_{2}$ correspondantes sont absolument continues l'une par rapport à l'autre si et seulement si le produit infini

$$
\prod_{k=1}^{\infty} \int_{0}^{+\infty} \sqrt{d q_{k, 1} d q_{k, 2}}
$$

est convergent, c'est-à-dire (on remarquera que, d'après l'inégalité de Cauchy-Schwarz, chacun des termes dans le produit infini est inférieur à 1) qu'il est non nul. De plus, si ce produit infini est divergent, alors les mesures $\mu_{1}$ et $\mu_{2}$ sont étrangères : il existe un ensemble A de $\mu_{1}$-mesure 1 et de $\mu_{2}$-mesure 0 .

4 e SÉRIE - TOME $46-2013-$ No $^{\circ}$ 
Ce critère garantit que pour « la plupart» des choix $\alpha_{k}, d p_{k}$, les mesures obtenues sont mutuellement étrangères : par exemple, si on choisit

$$
d p_{k, 1}=d p_{k, 2}=1_{r>0} \sqrt{\frac{2}{\pi}} e^{-\frac{r^{2}}{2}} d r
$$

un calcul simple [13, Appendix B.1] montre que le produit (C.8) est non nul si et seulement si

$$
\alpha_{k, 1}=0 \Leftrightarrow \alpha_{k, 2}=0 \text { et } \sum_{k}\left(\frac{\alpha_{k, 1}}{\alpha_{k, 2}}-1\right)^{2}<+\infty .
$$

\section{C.3. Densité du support}

Proposition C.2. - On suppose que les mesures $d p_{k}$ chargent tous les ouverts de l'intervalle $] 0,+\infty\left[\right.$, que $\left\|\left(\alpha_{k}\right)\right\|_{s}<+\infty$, et que tous les coefficients $\alpha_{k}$ sont non nuls. Alors le support de la mesure $\mathbb{P}$ associée est $H^{s}(M)$ :

$$
\forall v \in H^{s}(M), \forall \epsilon>0, \mathbb{P}\left(\left\{u \in H^{s}(M) ;\|u-v\|<\epsilon\right\}\right)>0 .
$$

On renvoie à [13, Appendix B.2] pour une preuve de ce résultat dans un cadre très légèrement différent.

\section{C.4. Estimées de grandes déviations}

Nous allons démontrer des estimées de grandes déviations pour les mesures sur l'espace $H^{s}(M)$. On notera $\langle t\rangle=\left(1+t^{2}\right)^{1 / 2}$.

Proposition C.3. - Soit $\mathbb{P} \in \mathcal{M}_{s}$. On suppose que la suite $p_{k}$ utilisée pour construire $\mathbb{P}$ vérifie l'hypothèse $\left(H_{\gamma}\right)$ de la page 955 , pour $\gamma>0$. On suppose aussi qu'il existe $D>0$ tel que pour tout $k$ assez grand on ait $a_{k+1}-a_{k} \geq D$. Alors il existe $D_{0}$ tel que, pour $D \geq D_{0}$, tout $2 \leq p<+\infty$ et tout $\delta>\frac{1}{p}$, il existe $c>0$ tel que

$$
\begin{array}{r}
\mathbb{P}\left(\left\{u=\sum_{k} u_{k} ;\|u\|_{H^{s}}>\lambda\right\}\right) \leq 2 e^{-c\left(\frac{\lambda}{\left\|\alpha_{k}\right\|_{s}}\right)^{\frac{\gamma}{\gamma+1}}} \\
\mathbb{P}\left(\left\{u=\sum_{k} u_{k} ;\left\|\langle t\rangle^{-\delta} \cos (t \sqrt{-\boldsymbol{\Delta}}) u\right\|_{W^{s, p}(\mathbb{R} \times M)}>\lambda\right\}\right) \leq 2 e^{-c\left(\frac{\lambda}{\left\|\alpha_{k}\right\|_{s}}\right)^{\frac{\gamma}{\gamma+1}}} \\
\mathbb{P}\left(\left\{u=\sum_{k} u_{k} ;\left\|\langle t\rangle^{-(\delta+1)} \frac{\sin (t \sqrt{-\boldsymbol{\Delta}})}{\sqrt{-\boldsymbol{\Delta}}} u\right\|_{W^{s+1, p}(\mathbb{R} \times M)}>\lambda\right\}\right) \leq 2 e^{-c\left(\frac{\lambda}{\left\|\alpha_{k}\right\|_{s}}\right)^{\frac{\gamma}{\gamma+1}}}
\end{array}
$$

Démonstration. - Des résultats similaires apparaissent dans un cadre légèrement différent dans [12]. Les démonstrations de ces trois estimées sont essentiellement identiques (la première n'ayant pas de dépendance en temps, tandis que les deux dernières ne diffèrent que par le comportement de la solution des ondes correspondant à la donnée initiale de fréquence 0). Nous nous limiterons donc à démontrer (C.10). On a $e^{i t \sqrt{-\Delta}} u=\sum_{k} e^{i t \sqrt{-\Delta}} u_{k}$.

Lemme C.4. - Il existe $C, c>0$ tels que pour tous $(x, t) \in \mathbb{R}_{t} \times M$

$$
\nu_{k}\left(\left|e^{i t \sqrt{-\Delta}} u_{k}\right|(x, t)>\lambda\right) \leq C e^{-c\left(\frac{\lambda}{\alpha_{k}}\right)^{\frac{2 \gamma}{\gamma+2}}} .
$$


En effet, quitte à remplacer la base orthonormale de $E_{k},\left(e_{n}\right)$ par $e^{i t \omega_{n}} e_{n}$, on se ramène au cas $t=0$. Si $D_{0}$ est assez grand, on peut utiliser le lemme 2.2, avec $h_{k}=a_{k}^{-1}$, donc

$$
\begin{aligned}
\nu_{k}\left(\left|u_{k}\right|(x)>\lambda\right) & =\int_{0}^{+\infty} \int_{z \in S_{k}} 1_{r\left|z \cdot b_{x, h_{k}}\right|>\lambda} d z d q_{k}(r) \\
& =\int_{0}^{+\infty} P_{h_{k}}\left(\left\{u ;|u(x)| \geq \frac{\lambda}{r}\right\}\right) d q_{k}(r) \leq \int_{0}^{+\infty} e^{-c_{2}\left(\frac{\lambda}{r \alpha_{k}}\right)^{2}} d p_{k}(r) .
\end{aligned}
$$

D'après l'hypothèse $H_{\gamma}$, on conclut si $\gamma=+\infty$ tandis que, si $\gamma<+\infty$, on obtient

$$
\nu_{k}\left(\left|u_{k}\right|(x)>\lambda\right) \leq e^{-c_{2}\left(\frac{\lambda}{\alpha_{k} \rho}\right)^{2}}+\int_{\rho}^{+\infty} d p_{k}(r) \leq e^{-c_{2}\left(\frac{\lambda}{\alpha_{k} \rho}\right)^{2}}+C e^{-c \rho^{\gamma}}
$$

qu'on optimise en choisissant $\rho=\left(\lambda / \alpha_{k}\right)^{2 /(\gamma+2)}$, ce qui donne le lemme C.4.

Lemme C.5. - Soient $\left(u_{k}\right)$ des variables aléatoires indépendantes, à valeurs réelles et de moments impairs tous nuls. Alors pour tout $q \in \mathbb{N}^{*}$,

$$
\mathbb{E}\left(\left(\sum_{k} u_{k}\right)^{2 q}\right) \leq q^{q} \mathbb{E}\left(\left(\sum_{k}\left(u_{k}\right)^{2}\right)^{q}\right) .
$$

Démonstration. - On s'inspire de la preuve classique des inégalités de Khintchine (voir par exemple [26, Théorème 4.6]). On calcule

$$
\mathbb{E}\left(\left(\sum_{k \leq K} u_{k}\right)^{2 q}\right)=\sum_{\alpha_{1}+\cdots+\alpha_{K}=2 q} \frac{(2 q) !}{\alpha_{1} ! \cdots \alpha_{K} !} \mathbb{E}\left(u_{1}^{\alpha_{1}} \cdots u_{K}^{\alpha_{k}}\right) .
$$

En utilisant l'indépendance et l'annulation des moments, on remarque que, dans la somme ci-dessus, les seuls termes non nuls sont ceux pour lesquels tous les $\alpha_{i}$ sont pairs, soit

$$
\mathbb{E}\left(\left(\sum_{k \leq K} u_{k}\right)^{2 q}\right)=\sum_{\beta_{1}+\cdots+\beta_{K}=q} \frac{(2 q) !}{\left(2 \beta_{1}\right) ! \cdots\left(2 \beta_{K}\right) !} \mathbb{E}\left(u_{1}^{2 \beta_{1}} \cdots u_{K}^{2 \beta_{k}}\right) .
$$

Comme $(2 \beta) ! \geq 2^{\beta} \beta$ ! et $(2 q) ! \leq 2^{q} q ! q^{q}$, on obtient

(C.13)

$$
\begin{aligned}
\mathbb{E}\left(\left(\sum_{k \leq K} u_{k}\right)^{2 q}\right) \leq \frac{1}{2^{q}} \sum_{\beta_{1}+\cdots+\beta_{K}=q} \frac{(2 q) !}{\left(\beta_{1}\right) ! \cdots\left(\beta_{K}\right) !} \mathbb{E}\left(u_{1}^{2 \beta_{1}} \cdots u_{K}^{2 \beta_{k}}\right) \\
=\frac{(2 q) !}{2^{q} q !} \sum_{\beta_{1}+\cdots+\beta_{K}=q} \frac{q !}{\left(\beta_{1}\right) ! \cdots\left(\beta_{K}\right) !} \mathbb{E}\left(u_{1}^{2 \beta_{1}} \cdots u_{K}^{2 \beta_{k}}\right) \leq q^{q} \mathbb{E}\left(\left(\sum_{k}\left(u_{k}\right)^{2}\right)^{q}\right) .
\end{aligned}
$$


On peut maintenant conclure la preuve de la proposition C.3 : pour $(x, t)$ fixés, on a, d'après le lemme C.5

(C.14)

$$
\begin{aligned}
\left\|\cos (t \sqrt{-\boldsymbol{\Delta}}) \sum_{k} u_{k}\right\|_{L^{2 q}(d \mathbb{P})} & \leq \sqrt{q}\left\|\left(\sum_{k}\left|\cos (t \sqrt{-\boldsymbol{\Delta}}) u_{k}\right|^{2}\right)^{1 / 2}\right\|_{L^{2 q}(d \mathbb{P})} \\
& \leq \sqrt{q}\left(\sum_{k}\left\|\left|\cos (t \sqrt{-\boldsymbol{\Delta}}) u_{k}\right|^{2}\right\|_{L^{q}(d \mathbb{P})}\right)^{1 / 2} \\
& =\sqrt{q}\left(\sum_{k}\left\|\cos (t \sqrt{-\boldsymbol{\Delta}}) u_{k}\right\|_{L^{2 q}(d \mathbb{P})}^{2}\right)^{1 / 2} \\
& \leq \sqrt{q}\left(\sum_{k}\left(\int_{0}^{+\infty} 2 q \lambda^{2 q-1} P_{k}\left(\left|\cos (t \sqrt{-\boldsymbol{\Delta}}) u_{k}\right|(x, t)>\lambda\right) d \lambda\right)^{\frac{1}{q}}\right)^{1 / 2} \\
& \leq C \sqrt{q}\left(q \frac{\gamma+2}{\gamma}\right)^{\frac{1}{2 q}}\left\|\left(\alpha_{k}\right)\right\|_{\ell^{2}} \Gamma^{\frac{1}{2 q}}\left(q \frac{\gamma+2}{\gamma}\right) \\
& \leq C q^{\frac{\gamma+1}{\gamma}}\left\|\left(\alpha_{k}\right)\right\|_{\ell^{2}}
\end{aligned}
$$

où, dans l'avant-dernière inégalité, on a utilisé le lemme C.4 et, dans la dernière, la formule de Stirling pour majorer la fonction $\Gamma$.

Finalement, on obtient que pour tous $2 \leq p \leq 2 q<+\infty$

$$
\begin{aligned}
\left\|\langle t\rangle^{-\delta} \cos (t \sqrt{-\boldsymbol{\Delta}}) u\right\|_{L^{2 q}\left((d \mathbb{P}) ; L^{p}(\mathbb{R} \times M)\right)} & \leq\left\|\langle t\rangle^{-\delta} \cos (t \sqrt{-\boldsymbol{\Delta}}) u\right\|_{L^{p}\left((\mathbb{R} \times M) ; L^{2 q}(d \mathbb{P})\right)} \\
& \leq C\left\|\alpha_{k}\right\|_{l^{2}} q^{\frac{\gamma+1}{\gamma}}\left\|\langle t\rangle^{-\delta}\right\|_{L^{p}(\mathbb{R} \times M)} \\
& \leq C q^{\frac{\gamma+1}{\gamma}}\left\|\alpha_{k}\right\|_{l^{2}} .
\end{aligned}
$$

Par l'inégalité de Tchebitchev on obtient

$$
\mathbb{P}\left(\left\{u ;\left\|\langle t\rangle^{-\delta} \cos (t \sqrt{-\boldsymbol{\Delta}}) u\right\|_{L^{p}\left(\mathbb{R}_{t} \times M\right)}>\lambda\right\}\right) \leq\left(\frac{C q^{\frac{\gamma+1}{\gamma}}\left\|\alpha_{k}\right\|_{l^{2}}}{\lambda}\right)^{2 q}
$$

et on conclut, et le choix $q=\frac{1}{e}\left(\frac{\lambda}{\left\|\alpha_{k}\right\|_{l^{2}}}\right)^{\frac{\gamma}{\gamma+1}} \geq 2$ si $\frac{\lambda}{C\left\|\alpha_{k}\right\|_{l^{2}}}$ est assez grand,

$$
\mathbb{P}\left(\left\{u ;\left\|\langle t\rangle^{-\delta} \cos (t \sqrt{-\boldsymbol{\Delta}}) u\right\|_{L^{p}\left(\mathbb{R}_{t} \times M\right)}>\lambda\right\}\right) \leq e^{-c\left(\frac{\lambda}{\left\|\alpha_{k}\right\|_{l^{2}}}\right)^{\frac{\gamma}{\gamma+1}}} .
$$

Enfin, si $\frac{\lambda}{\left\|\alpha_{k}\right\|_{l^{2}}}$ est borné, quitte à diminuer $c$, on a $1 \leq 2 e^{-c\left(\frac{\lambda}{\left\|\alpha_{k}\right\|_{l^{2}}}\right) \frac{\gamma}{\gamma+1}}$, ce qui termine la preuve de la proposition C.3 dans le cas $s=0$. Le cas général s'en déduit en remarquant que $\|v\|_{W^{s, p}(\mathbb{R} \times M)} \sim\left\|\left\langle\left|D_{t}\right|+\sqrt{-\boldsymbol{\Delta}}\right\rangle^{s} v\right\|_{L^{p}(\mathbb{R} \times M)}$. 


\section{RÉFÉRENCES}

[1] N. Anantharaman, Spectral deviations for the damped wave equation, Geom. Funct. Anal. 20 (2010), 593-626.

[2] M. Asch, G. Lebeau, The spectrum of the damped wave operator for a bounded domain in $\mathbf{R}^{2}$, Experiment. Math. 12 (2003), 227-241.

[3] A. Ayache, N. Tzvetkov, $L^{p}$ properties for Gaussian random series, Trans. Amer. Math. Soc. 360 (2008), 4425-4439.

[4] C. Bardos, G. Lebeau, J. Rauch, Sharp sufficient conditions for the observation, control, and stabilization of waves from the boundary, SIAM J. Control Optim. 30 (1992), 1024-1065.

[5] A. L. Besse, Manifolds all of whose geodesics are closed, Ergebnisse Math. Grenzg. 93, Springer, 1978.

[6] M. D. Blair, H. F. Smith, C. D. Sogge, Strichartz estimates for the wave equation on manifolds with boundary, Ann. Inst. H. Poincaré Anal. Non Linéaire 26 (2009), 1817-1829.

[7] D. D. Bleecker, Nonperturbative conformal quantum gravity, Classical Quantum Gravity 4 (1987), 827-849.

[8] J. Bourgain, Eigenfunction bounds for the Laplacian on the $n$-torus, Int. Math. Res. Not. 1993 (1993), 61-66.

[9] J. Bourgain, Moment inequalities for trigonometric polynomials with spectrum in curved hypersurfaces, Israel J. Math. 193 (2013), 441-458.

[10] J. Bourgain, Z. Rudnick, Restriction of toral eigenfunctions to hypersurfaces and nodal sets, Geom. Funct. Anal. 22 (2012), 878-937.

[11] N. Burq, G. Lebeau, F. Planchon, Global existence for energy critical waves in 3-D domains, J. Amer. Math. Soc. 21 (2008), 831-845.

[12] N. Burq, N. Tzvetkov, Random data Cauchy theory for supercritical wave equations. I. Local theory, Invent. Math. 173 (2008), 449-475.

[13] N. Burq, N. Tzvetkov, Probabilistic well-posedness for the cubic wave equation, prépublication arXiv:1103.2222.

[14] M. Dimassi, J. Suöstrand, Spectral asymptotics in the semi-classical limit, Lecture Note Series 268, Cambridge University Press, 1999.

[15] I. Gallagher, P. Gérard, Profile decomposition for the wave equation outside a convex obstacle, J. Math. Pures Appl. 80 (2001), 1-49.

[16] E. Grosswald, Representations of integers as sums of squares, Springer, 1985.

[17] V. Guillemin, Some classical theorems in spectral theory revisited, in Seminar on singularities of solutions of linear partial differential equations (Inst. Adv. Study, Princeton, N.J., 1977/78), Ann. of Math. Stud. 91, Princeton Univ. Press, 1979, 219-259.

[18] L. Hörmander, The spectral function of an elliptic operator, Acta Math. 121 (1968), 193-218.

[19] L. Hörmander, The analysis of linear partial differential operators. IV, Grund. Math. Wiss. 275, Springer, 1985.

$4^{\mathrm{e}}$ SÉRIE - TOME $46-2013-\mathrm{N}^{\circ} 6$ 
[20] O. Ivanovici, Counterexamples to Strichartz estimates for the wave equation in domains, Math. Ann. 347 (2010), 627-673.

[21] V. IVRII, Microlocal analysis and precise spectral asymptotics, Springer Monographs in Math., Springer, 1998.

[22] S. Kakutani, On equivalence of infinite product measures, Ann. of Math. 49 (1948), 214-224.

[23] Y. KIFER, Large deviations in dynamical systems and stochastic processes, Trans. Amer. Math. Soc. 321 (1990), 505-524.

[24] G. Lebeau, Équation des ondes amorties, in Algebraic and geometric methods in mathematical physics (Kaciveli, 1993), Math. Phys. Stud. 19, Kluwer Acad. Publ., 1996, 73-109.

[25] M. Ledoux, The concentration of measure phenomenon, Mathematical Surveys and Monographs 89, Amer. Math. Soc., 2001.

[26] D. Li, H. QuefFÉLeC, Introduction à l'étude des espaces de Banach, Cours Spécialisés 12, Soc. Math. France, 2004.

[27] A. Martinez, An introduction to semiclassical and microlocal analysis, Universitext, Springer, 2002.

[28] F. Morgan, Measures on spaces of surfaces, Arch. Rational Mech. Anal. 78 (1982), 335-359.

[29] B. Shiffman, S. Zelditch, Random polynomials of high degree and Levy concentration of measure, Asian J. Math. 7 (2003), 627-646.

[30] J. SuöstRAND, Asymptotic distribution of eigenfrequencies for damped wave equations, Publ. Res. Inst. Math. Sci. 36 (2000), 573-611.

[31] H. F. Smith, C. D. Sogge, On the $L^{p}$ norm of spectral clusters for compact manifolds with boundary, to appear in Acta Math.

[32] C. D. Sogge, Concerning the $L^{p}$ norm of spectral clusters for second-order elliptic operators on compact manifolds, J. Funct. Anal. 77 (1988), 123-138.

[33] C. D. Sogge, Eigenfunction and Bochner Riesz estimates on manifolds with boundary, Math. Res. Lett. 9 (2002), 205-216.

[34] M. Struwe, On uniqueness and stability for supercritical nonlinear wave and Schrödinger equations, Int. Math. Res. Not. 2006 (2006), Art. ID 76737, 14.

[35] N. Tzvetkov, Invariant measures for the defocusing nonlinear Schrödinger equation, Ann. Inst. Fourier (Grenoble) 58 (2008), 2543-2604.

[36] N. Tzvetkov, Riemannian analogue of a Paley Sygmund theorem, Séminaire É.D.P., exposé $\mathrm{n}^{\circ} \mathrm{XV}$ (2008-2009), 1-14.

[37] J. M. VAnder Kam, $L^{\infty}$ norms and quantum ergodicity on the sphere, Int. Math. Res. Not. 1997 (1997), 329-347.

[38] S. Zelditch, A random matrix model for quantum mixing, Int. Math. Res. Not. 1996 (1996), 115-137.

[39] S. Zelditch, Real and complex zeros of Riemannian random waves, in Spectral analysis in geometry and number theory, Contemp. Math. 484, Amer. Math. Soc., 2009, 321-342. 
[40] M. Zworski, Semiclassical analysis, Graduate Studies in Math. 138, Amer. Math. Soc., 2012.

(Manuscrit reçu le 30 novembre 2011; accepté, après révision, le 18 janvier 2013.)

\author{
Nicolas BURQ \\ Laboratoire de Mathématiques d'Orsay \\ Université Paris-Sud \\ UMR 8628 du CNRS \\ Bâtiment 425 \\ 91405 Orsay Cedex, France \\ E-mail: nicolas.burq@gmail.com \\ Gilles Lebeau \\ Département de Mathématiques \\ Université de Nice Sophia-Antipolis \\ Parc Valrose \\ 06108 Nice Cedex 02, France \\ et Institut Universitaire de France \\ E-mail: lebeau@unice.fr
}

